\title{
Methods to Design Microstrip Antennas for Modern Applications
}

\author{
K. Siakavara \\ Aristotle University of Thessaloniki \\ Dept. of Physics, Radiocommunications Laboratory, \\ 54124 Thessalonik, \\ Greece
}

\section{Introduction}

The evolution of modern wireless communications systems has increased dramatically the demand for antennas, capable to be embedded in portable, or not, devices which serve a wireless land mobile or terrestrial-satellite network. With time and requirements, these devices become smaller in size and hence the antennas required for transmit and receive signals have also to be smaller and lightweight. As a matter of fact, microstrip antennas can meet these requirements. As they are lightweight and have low profile it is feasible them to be structured conformally to the mounting hosts. Moreover, they are easy fabricated, have low cost and are easy integrated into arrays or into microwave printed circuits. So, they are attractive choices for the above mentioned type of applications.

For all that, the design of a microstrip antenna is not always an easy problem and the antenna designer is faced with difficulties coming from a) the inherent disadvantages of a printed resonant antenna element, for example the narrow impedance bandwidth, and b) the various requirements of the specific applications, which concern the operation of the radiating element, and can not be satisfied by a printed scheme with an ordinary configuration. For example, it would be demanded, the microstrip element to have gain characteristics that potentially incommensurate to its size or/and frequency bandwidth greater than the element could give, taking into account that it operates as a resonant cavity. Moreover, the rapid development in the field of Land Mobile Telephony as well as in the field of Wireless Local Area Networks(WLANs) demands devices capable to operate in more than one frequency bands. So the design of a printed antenna with intend to conform to multiple communications protocols, for example the IEEE $802.11 \mathrm{~b} / \mathrm{g}$, in the band of $2.4 \mathrm{GHz}$, and the IEEE $802.11 \mathrm{a}$ at $5.3 \mathrm{GHz}$ and $5.8 \mathrm{GHz}$, would be a difficult task but at the same time a challenge for the designer. Counting in the above the possibility the device, and so the antenna, to serve terrestrial and also satellite navigation systems the problem of the antenna design is even more complicated.

In this chapter techniques will be analysed, to design microstrip antennas that combine the attributes mentioned above which make them suitable for modern communications applications. Specific examples will be also presented for every case. 


\section{Bandwidth enhancement and multiband operation}

\subsection{Bandwidth}

Conventional microstrip antennas have a conducting patch printed on a grounded dielectric substrate and operate as resonant cavity elements. This operation leads inherently to narrow impedance bandwidth which is a barrier for microstrip antennas applications in wireless communications. Moreover in many of these applications, as further requirement would be a multi-frequency operation. So, the enhancement of the bandwidth and the achievement of multifrequency operation are major challenges for the antenna designer and many techniques have been proposed for this purpose.

The fundamental definition of the bandwidth of an antenna is the difference between the upper and lower frequencies of operation $\left(f_{H}\right.$ and $f_{L}$ respectively)

$$
\mathrm{bw}=\mathrm{f}_{\mathrm{H}}-\mathrm{f}_{\mathrm{L}}
$$

For all that, the spectrum managers often use a variety of different bandwidth definitions, including fractional or percent bandwidth. These measures of relative bandwidth require the calculation of a central frequency, which is either the arithmetic or geometric average of the upper and lower frequencies. The center frequency is defined as the arithmetic average of the upper and lower frequencies

$$
\mathrm{f}_{\mathrm{C}}=\frac{\mathrm{f}_{\mathrm{H}}+\mathrm{f}_{\mathrm{L}}}{2}
$$

An arithmetic average yields the central frequency when frequency is considered on a linear scale. An alternate definition of center frequency involves the geometric average

$$
\mathrm{f}_{\mathrm{C}}=\sqrt{\mathrm{f}_{\mathrm{H}} \mathrm{f}_{\mathrm{L}}}
$$

The geometric average yields the center frequency when frequency is considered on a logarithmic scale and is less commonly used. So the arithmetic average should be assumed unless otherwise is specified.

The fractional bandwidth of a system is the ratio of the bandwidth to the center frequency(either the geometric or the arithmetic definition is used)

$$
\mathrm{BW}=\frac{\mathrm{bw}}{\mathrm{f}_{\mathrm{C}}}
$$

Alternatively, fractional bandwidth may be defined on a percentage basis

$$
\mathrm{BW} \%=\frac{\mathrm{bW}}{\mathrm{f}_{\mathrm{C}}} 100 \%
$$

Since the geometric definition of center frequency always yields a frequency smaller than the arithmetic average, fractional bandwidths calculated using the geometric definition are always larger than the arithmetic ones. So, the designer of the antenna has to pay attention on which definition is used. Although specific limit values of BW in order an antenna to be considered as broadband do not exist, criteria to rank an antenna as ultra-wideband have been enacted. In accordance with these criterions an antenna is characterized as UWB if its 
fractional bandwidth (based on the arithmetic central frequency) exceeds the value of 0.25(Defense Advanced Research Projects Agency_DRPA) or the value of 0.2(Federal Communications Commission_FCC).

\subsection{Bandwidth enhancement}

The impedance frequency bandwidth of a microstrip antenna depends primarily on both the thickness and the dielectric permittivity of the substrate. A thick substrate with a low dielectric permittivity can increase the bandwidth of the printed patch. Both these selections could be a solution of the problem of bandwidth enhancement if the thickness of the substrate did not a)pose difficulties in integration of the antenna with other microwave circuits, and b)cause some other problems such as the surface wave propagation and the large inductive image part of the input impedance of the antenna, which makes its resonance unfeasible. Thus, a reasonable thickness should be considered in the selection of substrate and the bandwidth would be enhanced using additional techniques. The most common and effective of them, are: a) the loading of the surface of the printed element with slots of appropriate shape b)the texturing of narrow or wide slits at the boundary of the microstrip patch . Other effective techniques used for the enhancement of the bandwidth is the utilization of a)stacked, shorted or not patches, [1]-[3] and b) extra microstrip resonators [4]. The technique of stacked patches is based on the fact that the bandwidth is in general proportional to the antenna volume measured in wavelengths but at the same time a relatively large volume is a disadvantage for many applications. The utilization of additional parasitic patches of different size directly- or gap-coupled to the main patch is an effective method but results to an increased antenna size which would also be undesired. Superior to these methods are the techniques of slot loading or texturing the patches by slits because they ensure the small size and the low profile of the antennas.

\subsubsection{Slot loaded patches}

The slot loading is made by two ways. By one of them, slots are embedded in the printed patch. Their dimensions and positions are properly selected in order to the first two broadside-radiation modes of the patch be perturbed such that their resonance frequencies get close to each other to form a wide impedance bandwidth. The slots would be of various shapes as, toothbrush(Fig. 1a)[5], double bend (Fig. 1b)[6] , cross (Fig. 1c) [7], or U-shape.

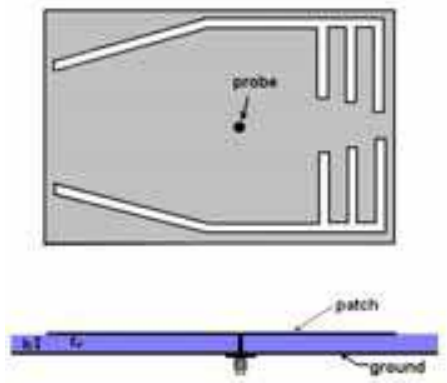

(a)

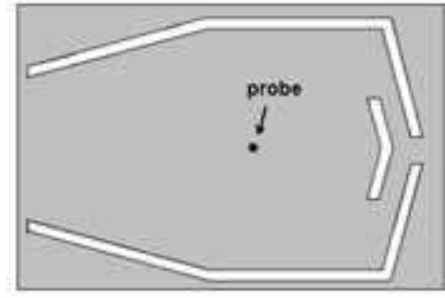

(b)

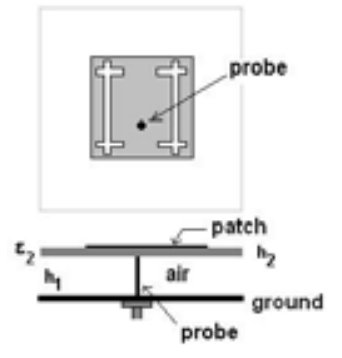

(c)

Fig. 1. Microstrip antennas loaded with slots of various shapes : a) 'toothbrush' b) 'double bend c)cross 
The microstrip elements etched with a U-slot could be rectangular or triangle patches (Fig. 2)and are perhaps the most popular among the antennas of this type[8]-[15], as they have been proved to be versatile radiating elements: they can be designed not only for wideband applications but also for dual- and triple-band as well as for circular polarization operation. It has been found that the U-slot loaded patch can provide impedance bandwidth in excess of $30 \%$ for an air substrate thickness of $0.08 \lambda$ o and in excess of $20 \%$ for material substrate of similar thickness [16].

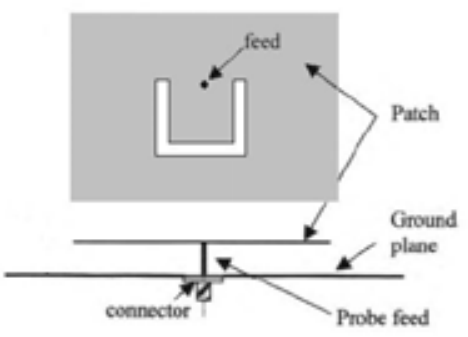

(a)

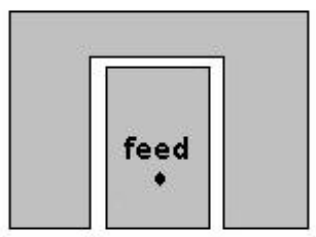

(b)

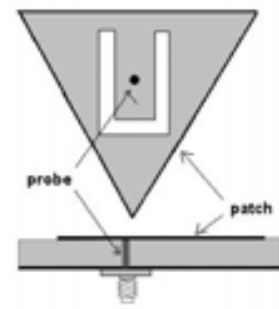

(c)

Fig. 2. The geometry of the U-slot patch antenna.

The U-slot is consisted of two parallel vertical rectangular slots and an horizontal rectangular one. The parameters that affect the broadband performance of the patch antenna are the slot length and width and the position of the slot. It is noted that the slot width should be small relative to the slot length and the higher resonant mode is sensitive to the length variation of the horizontal slot, whereas the lower resonant mode strongly depends on the perimeter of the U-slot. Unfortunately mathematical formulas, even empirical, by which the accurate geometry of the structure patch-U-slot could be find in order the antenna to have a pre-specified operation, are not available yet. Empirical formulas introduced in the past[10] are limited to electrically thin substrates which lead to small bandwidth. Thus the antenna designers should adjust the dimensions and the position of the slot by iterative trials or, potentially, by employment of an evolutionary technique of prediction and optimization as in $\$ 4$ is discussed.

An alternative version of the U-slot patch is the half U-slot patch Fig3a, which maintain an impedance bandwidth similar to that of a full U-slot patch having the further advantage of the smaller size[17]-[18]. This is due to the fact that the electrical current distribution is symmetrical along the line of symmetry of the full U-slot printed element. So, removing half of the patch does not appreciably affect the current paths and, hence, the resonant behavior of the structure. Moreover, a shorting wall (Fig. 3b) can be integrated to reduce the size of the full U-slot [19]-[20] or the half -U-slot patch antenna[17]- [21]. These small-size wide bandwidth designs are particularly suitable for handset devices.

\subsubsection{Slit loaded patches}

By slit cutting at the boundaries, instead of slot cutting on the surface, of the patch, similar broadband operation can be obtained. The resulting configuration is E-shaped or suchlike and the design can be applied to the antennas with rectangular, circular or triangular patches(Fig. 4)[22]- [24]. 


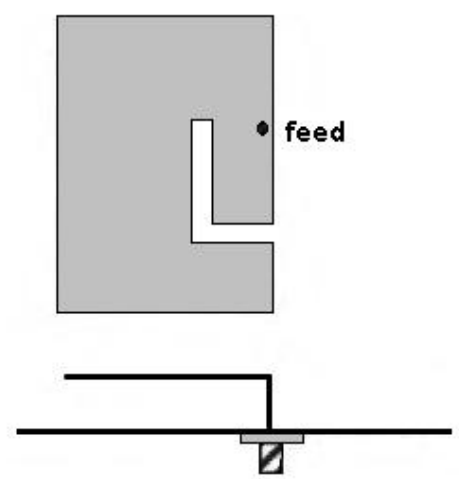

(a)

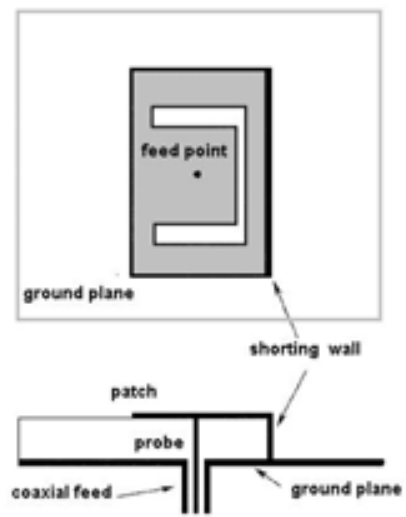

(b)

Fig. 3. Patterns of a)Half U-slot loaded patch b) U-slot loaded patch with shorting wall

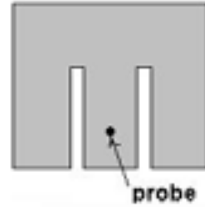

(a)

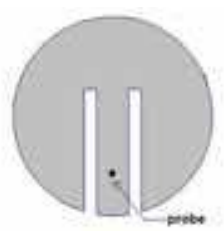

(b)

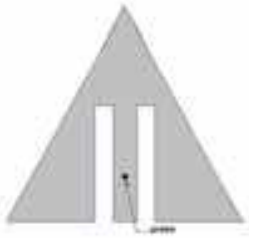

(c)

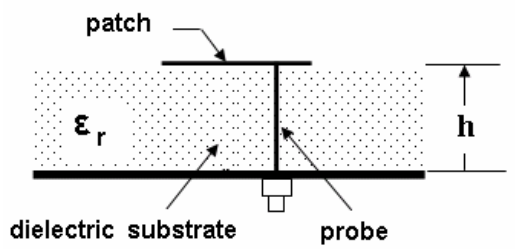

Fig. 4. The geometry of slit loaded microstrip antennas

The wideband performance of the slit loaded patch is based, similarly to the method of slotloading, on the excitation of more than one adjacent resonant modes. Moreover the presence of the slits in the vicinity of the feeding probe could add a capacitive load at the input impedance of the patch. This capacitive load could effectively contribute to the resonance of the patch because can counteract the inductive part of the probe's input impedance. It is noticed that this inductive part would inevitably be large if a thick substrate is chosen for wideband operation. So, the insertion of slits enhances by two ways the width of the operation band, and it has been reported that bandwidth greater than $25 \%$ can be achieved. The width of the frequency band of the antenna can be controlled by the slits' length and width and the slits' position. The slits divide the patch in three or more parts and at each one corresponds an equivalent circuit of resonance(Fig. 5)[25]-[26]. In order the three regions of resonance to overlap, forming a unified wideband range of operation, the slits could potentially have unequal size. 


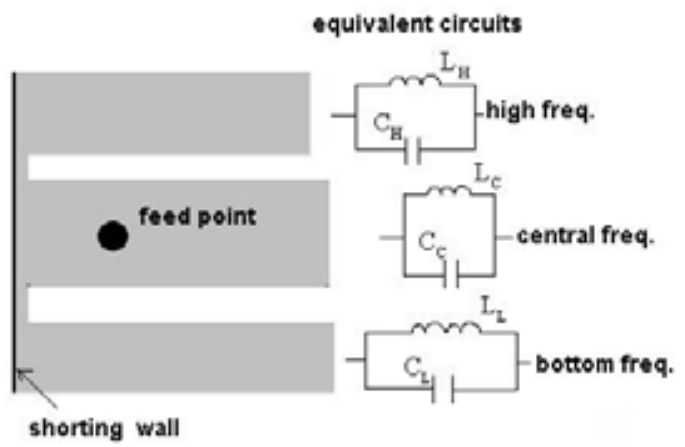

Fig. 5. The structure and the equivalent circuits of E-shaped printed antenna

A lightly different patch with as much bandwidth as the E-patch can be realized by configuring the slits in a way that results in a printed element with meander shape (fig 6) [27].
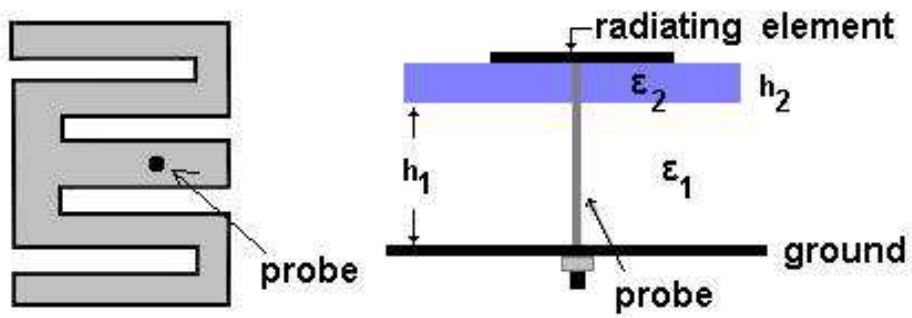

Fig. 6. Top and side view of the meander shaped printed antenna

Another slit loading process to obtain broad band operation is to create several slits at the boundary of the patch. This technique provides the patch with the additional advantage of a size smaller than that of the previous configurations. As an example a patch with five-couple staggered slits is presented in figure 7a [28]. A hybrid feed, inset feed combined with probe, is used for easy impedance matching. A quantitative analysis can prove the possibility to expand the operation bandwidth and reduce the antenna size. When the structure parameters of the antenna are selected properly, two $\mathrm{TM}_{10}$ modes can be excited simultaneously. The simple electrical current sketches of the two modes on the patch are shown in Fig. $7 \mathrm{~b}$. It can be found that the current paths of the modes overlap at the top portion of the patch whereas they occupy a different structure branch at the bottom portion of the patch.

In addition, the current path lengths are determined by the patch length and the slit depths. The asymmetric slit loading technique leads to different resonance lengths of the two $\mathrm{TM}_{10}$ modes. The two modes can be matched by adjusting the depth of the feed slits and the patch length because their input impedances vary with the inset feed position. Moreover due to the slits, the currents of the modes flow on meandering paths that ensure the required length for resonance at the modes' frequencies, whereas the entire length of the patch is smaller compared with that of ordinary rectangular microstrip antennas. Furthermore, the current paths of the $\mathrm{TM}_{10}$ modes are along the same direction, thus the radiation characteristics of the modes are similar, in accordance to the radiation theory. Due to the above mechanism of operation, the bandwidth and the size of the antenna could be controlled by the slit number and the geometry of the whole configuration. 

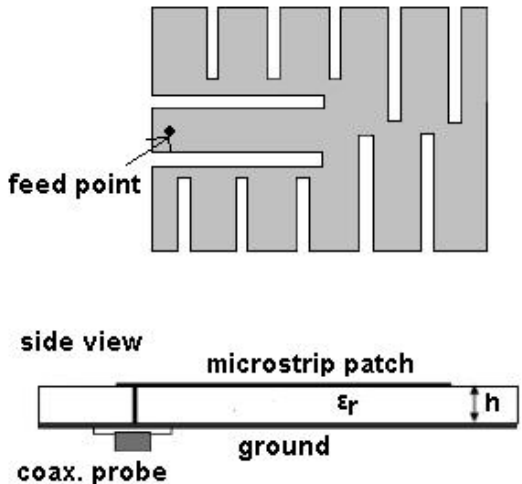

(a)

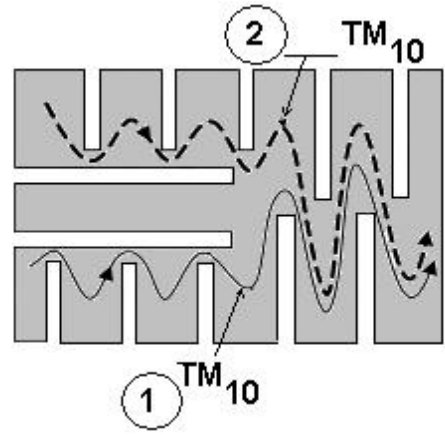

(b)

Fig. 7. a)Configuration of multi-slit loaded low profile microstrip antenna b)Current sketches of the two modes on the patch

In all cases of the slit loading, the slits play an important role to control the wideband behavior of the antenna. However to find the proper geometry of the textured patch is usually a difficult process because there are no mathematical formulas, thus, no prediction could be made. The whole process is iterative and is applied by simulations using a high frequency electromagnetic field simulation software. The set up of the process is decided by the designer. Usually the first step of the procedure is to select the dielectric substrate and design the initial unmodified patch. Then slots are etched and their position and geometry are iteratively adjusted to tune the required frequency and the bandwidth. The whole strategy potentially includes steps of simultaneous small variations of the patch dimensions and of the substrate with intend to control the frequency tuning and the bandwidth[24], [27]. Besides this strategy, which is substantially a trial and error one, Artificial Network Algorithms or Optimization techniques as Genetic Algorithms, Particle Swarm Optimization(PSO) or Differential Evolution could be applied(See §4). By these methods all the material and geometrical parameters of the antenna structure are simultaneously taken into account in every step of the design process.

\subsubsection{Ultra-Wideband microstrip antennas}

There are two criteria available for identifying when an antenna may be considered ultrawideband(UWB). One definition(by Defense Advanced Research Projects Agency report) requires a UWB to have fractional bandwidth greater than 0.25. An alternate and more recent definition, by Federal Communications Commission(FCC) places the limit at 0.2. Using fractional bandwidth (eq. 4), in mathematical form it is

$$
\mathrm{BW}=2 \frac{\mathrm{f}_{\mathrm{H}}-\mathrm{f}_{\mathrm{L}}}{\mathrm{f}_{\mathrm{H}}+\mathrm{f}_{\mathrm{L}}}= \begin{cases}0.25 & \text { DARPA } \\ 0.2 & \text { FCC }\end{cases}
$$

In the past, different types of small antennas meeting the above requirement have been investigated: resistively loaded pyramidal horn antennas, stacked patch antennas and tapered slot antennas or spiral-shaped antennas. Among the existing designs of printed structures, the ones that provide the required operational bandwidth while maintain 
sufficient radiation efficiency, simple design structure and low fabrication cost are: a) the spiral shaped antenna b)the tapered slot, especially the antipodal tapered, as the Vivaldi antennas and c) the bowtie shaped antennas.

\section{a. Spiral antennas}

Spiral antennas have been the subject of research for several decades and have emerged as leading candidates for applications requiring circularly polarized broadband antennas. Spirals can have a single arm or multiple arms and have been realized in microstrip form as well as in slot form. But spiral antennas have shortcomings as well. They require deeper lossy cavities and special attention to impedance transformer network design. Recently several designs of planar spiral antennas without complicated feeding networks were proposed. They are planar, easy to fabricate and do not need a matching network. The spiral could have annular(fig. 8a ) or rectangular(fig. 8b) shape. The microstrip Archimedean annular spiral antenna can be achieved by the traditional Archimedean spiral concept with a dielectric-substrate and a metal ground plane. The spiral configuration could increase the gain of the antenna. However the main lobe splits at the higher frequency band.

The radiation principle of this kind of microstrip configuration is based on the mirror concept. To reach the property of ultra-wide band, the height of the dielectric -slab and the value of the dielectric constant is very important. At the lower part of frequency band, the maximum radiation of the antenna can be found along the axis. By the rising of the frequency the front to back ratio increases and one-sided radiation can be achieved. The direction of the maximum radiation starts to deflect at a specific frequency, depending on the structural characteristics of the spiral. As the frequency rises further the direction deflects to both sides of the axis which is perpendicular to the antenna's surface. The decreasing of the radiation along the axis is explained as follows: The height of the dielectric-slab is related to the central frequency of the antenna, being about a quarter of the wavelength at this frequency, as it is defined inside the dielectric. So, at the higher frequency band the height tends to be one-half of the wavelength at the respective frequencies. In this case, the phase of the original signal, compared with that of the wave reflected by the the ground plane is just the opposite when it reaches the spiral plane. After the superposition of the signals, the radiation at the broadside decreases and the main lobe splits. A modification of the substrate is proposed in this case. A cylinder with a certain radius should be subtracted out from the center of the dielectric so as to improve the gain along the axial direction at higher frequency band. The physical thickness of the substrate in the hollow part corresponds to an electrical length smaller than that in the rest of the dielectric slab. The parameters of the hollow have proper values in order this length to be equal to $\lambda / 4$

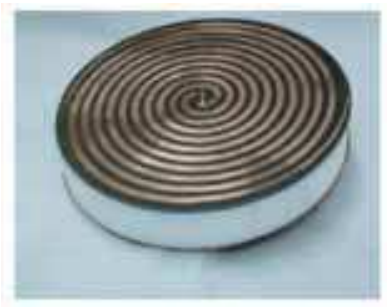

(a)

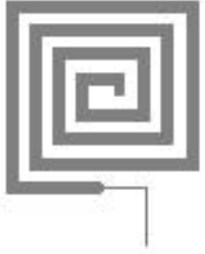

(b)

Fig. 8. a) Structural pattern of the microstrip circular spiral printed antenna b)Sketch of the rectangular spiral antenna 
instead of $\lambda / 2$. So, the condition for maximum radiation toward the direction perpendicular to the surface of the antenna is fulfilled. At the operation inside the wide frequency band, the effective region of the antenna at each frequency includes the annuluses of the Archimedean spiral whose perimeters are approximately equal to the respective wavelength. By this modification, the proper radius of the hollow cylinder and the proper dielectric constant of the substrate, we can lead the spiral configuration to a wide band operation, broadside radiation and a gain greater than that of the ordinary spiral structure.

\section{b. Vivaldi antenna}

Vivaldi antenna was invented by Gibson in 1979, and has been widely investigated and used in various applications due to its broad bandwidth, low cross polarization and high directivity[30]-[31]. It is a kind of traveling-wave planar antenna, guiding the wave from a narrower slotline to a wider slotline, which varies by exponential rule, where the wave is radiated out from the horn opening. Due to the non-uniform shape of the printed area, different parts are activated at different frequencies. In virtue of this performance the entire scheme can operate in a wide band of frequencies. However it is difficult to select the best parameters and variances to lead the antenna up to the best radiation performance.

The standard model of the Vivaldi antenna is shown in Fig. 9. There are more than ten parameters to be designed. Furthermore, one important part of the antenna is the microstrip-slotline transition feeding structure. This type of feeding is the most commonly used. Microstrip and slotline are deposited in the different sides of the substrate. The incident wave power can be coupled from the microstrip to slotline and then the power can be transmitted to the air through the exponential tapered parts. The impedance match at the coupling point is usually difficult. Three are the parameters that affect the radiation performance of the antenna: the microstrip-slotline transition magnitudes of strip stub, the slotline circle cavity and the gradual rates of the antenna. So, the design of the slotline structure, and the calculation of the antenna' s exponential tapered parts are the basic steps of the design.

The configuration of the exponential tapered part is governed by three parameters: a) the slotline hatch $(\mathrm{H}) \mathrm{b}$ )the antenna tapered length(L) and c) the exponential gradual (a) , as shown in Fig. 9. According to experience, the higher $\left(\mathrm{F}_{\mathrm{H}}\right)$ and lower $\mathrm{F}_{\mathrm{L}}$ cut-off frequencies determine the slotline hatch. Generally, the widest hatch $(\mathrm{H})$ is equal to $1.3 \lambda_{\mathrm{cL}}$, where $\lambda_{\mathrm{cL}}$ corresponds to the wavelength of the lower cut-off frequency; the narrowest hatch is equal to $0.2 \lambda_{\mathrm{cH}}$, where $\lambda_{\mathrm{cH}}$ corresponds to the wavelength of the higher cut-off frequency. The antenna tapered length $(\mathrm{L})$ is equal to $3 \sim 5$ times the wavelength of the central operation frequency.

The exponential gradual ratio can be determined by the exponential gradual curve

$$
\mathrm{y}=\mathrm{C}_{1} \mathrm{e}^{\mathrm{\alpha x}}+\mathrm{C}_{2}
$$

Where $C_{1}$ and $C_{2}$ can be calculated using $C_{1}=\frac{y_{2}-y_{1}}{e^{\alpha x_{2}}-e^{\alpha x_{1}}}$ and $C_{2}=\frac{y_{1} e^{\alpha x_{2}}-y_{2} e^{\alpha x_{1}}}{e^{\alpha x_{2}}-e^{\alpha x_{1}}}$

And $\left(\mathrm{x}_{1}, \mathrm{y}_{1}\right)$ and $\left(\mathrm{x}_{2}, \mathrm{y}_{2}\right)$ are the begin point and the end point of the exponential gradual curve.

A modified form of the traditional Vivaldi antenna is the dual exponentially tapered antipodal antenna(DETASA)[31]. It is realized by exponentially tapering both the inner and 


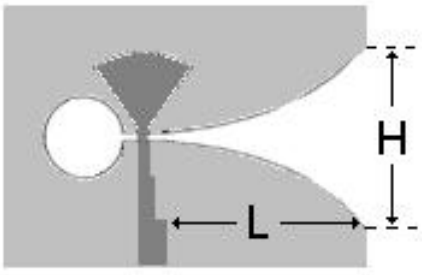

(a)

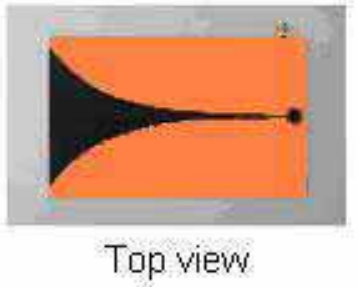

(b)

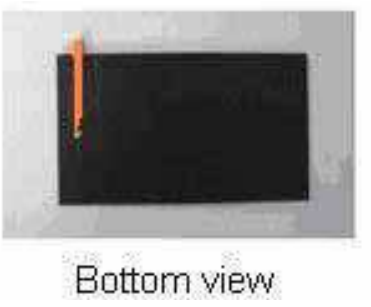

Bottom view

Fig. 9. Printed Vivaldi antenna a) the model b) the manufactured structure

the outer edges of the radiating flare and placing the mirrored flares on both sides of the substrate(Fig. 10a). It is a slow leaky end-fire traveling wave antenna. The electromagnetic wave travels down the gradually curved paths of the flares. As the separation between the flares increases, the wave becomes progressively weaker and radiates away. A wideband performance can be achieved using DETASA, in virtue of its inherently simple wideband transition from microstrip line to tapered slot flare through parallel strips.

The rule for the design of the radiating flare is described by equations $8 \mathrm{a}$ and $8 \mathrm{~b}$.

$$
\begin{gathered}
\mathrm{x}_{\text {inner }}= \pm\left[-\left(\mathrm{c}_{\mathrm{s}}+\mathrm{c}_{\mathrm{w}}\right) / 2+\mathrm{c}_{\mathrm{s}} \cdot \exp \left(\mathrm{k}_{\mathrm{s}} \mathrm{y}_{\text {inner }}{ }_{\mathrm{sft}}\right)\right] \\
\mathrm{x}_{\text {outer }}= \pm\left[-\left(\mathrm{c}_{\mathrm{s}}+\mathrm{c}_{\mathrm{w}}\right) / 2+\mathrm{c}_{\mathrm{w}} \cdot \exp \left(\mathrm{k}_{\mathrm{w}} \mathrm{y}_{\text {outer }}^{\text {sfo }}\right)\right]
\end{gathered}
$$

Where $\mathrm{x}_{\text {inner }}$ and $\mathrm{x}_{\text {outer }}$ are the horizontal distances from the inner and outer exponential edges to the center line of the antenna. $y_{\text {inner }}$ and $y_{\text {outer }}$ are the vertical distances from the edges to the bottom line of the antenna. The other parameters are obtained by the trial-and error optimization procedure using full-wave electromagnetic simulation or applying optimization techniques as Genetic Algorithms, Particle Swarm Optimization, etc.

In order to further extend the operational bandwidth and he radiation performance, a modification of DETASA is introduced by merging the exponential flare with a circular tapering termination before the edges reach the end of the substrate(Fig. 10b). It creates a longer path for the current flow making the antenna bandwidth greater and smoother. In order to avoid sharp gradient change between the exponential edges and the circular

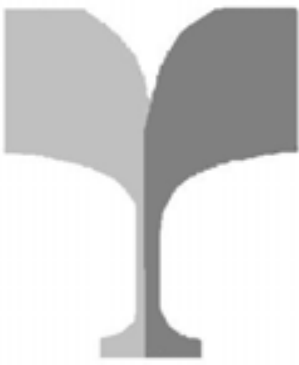

(a)

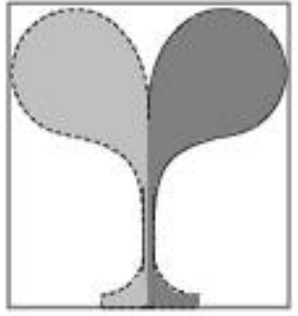

(b)

Fig. 10. Two Configurations of the Dual Exponential Antipodal Vivaldi Antenna 
tapering, optimization is performed to search for appropriate tapering parameters along the pre-fixed inner and outer exponential edges and to provide defining parameters for the antenna configuration. Extra spacing is kept between the flares and the edge of the substrate. This additional space helps to reduce ringing of the time-domain impulse response.

With respect to the feeding method of this type of UWB antennas, the transition from the feeding source to the radiation flares is designed by the high frequency transmission line theory. The exact theoretical analysis in detail, and the corresponding realization depend on the Vivaldi antenna configurations(traditional or modified).

\section{c) Bowtie printed antennas}

Another patch configuration that drives the antenna system to multifrequency operation is the bowtie shape. Bowtie microstrip antennas have become attractive candidates in the present day communication systems due to their size, that is smaller than the size of a conventional rectangular patch although they have similar characteristics and operation at the same frequency. The fundamental shape of a bowtie microstrip antenna is shown in fig. 11.

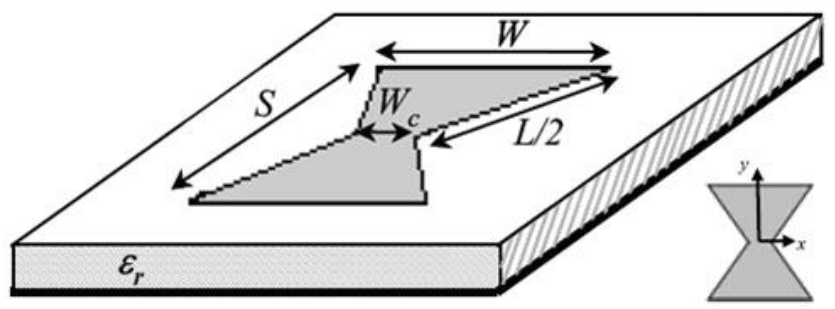

Fig. 11. Geometry of the bow-tie microstrip antenna

The bowtie printed object comes, substantially, from a rectangular patch via modification and the equations for the approximate calculation of the resonance frequency $f_{r}$ in terms of the geometrical and material parameter values are,[32]

$$
\mathrm{f}_{\mathrm{r}}=1.152 \frac{\mathrm{c}}{2 \sqrt{\varepsilon_{\text {eff }}} \mathrm{L}} \frac{\mathrm{L}}{2} \frac{\left[(\mathrm{W}+2 \Delta \mathrm{L})+\left(\mathrm{W}_{\mathrm{c}}+2 \Delta \mathrm{L}\right)\right]}{(\mathrm{W}+2 \Delta \mathrm{L})(\mathrm{S}+2 \Delta \mathrm{L})}
$$

Where

$$
\Delta \mathrm{L}=\frac{0.412 \mathrm{~h}\left(\varepsilon_{\mathrm{eff}}+0.3\right)\left(\frac{\mathrm{W}+\mathrm{W}_{\mathrm{c}}}{2 \mathrm{~h}}+0.262\right)}{\left[\left(\varepsilon_{\mathrm{eff}}-0.258\right)\left(\frac{\mathrm{W}+\mathrm{W}_{\mathrm{c}}}{2 \mathrm{~h}}+0.813\right)\right]}
$$

and

$$
\varepsilon_{\text {eff }}=\left(\frac{\varepsilon_{\mathrm{r}}+1}{2}\right)+\left(\frac{\varepsilon_{\mathrm{r}}-1}{2}\right)\left(\frac{24 \mathrm{~h}}{\mathrm{~W}+\mathrm{W}_{\mathrm{c}}}+1\right)^{-1 / 2}
$$


By altering the dimensions $\mathrm{W}_{\mathrm{c}}$ and $\mathrm{L}$ and keeping $\mathrm{W}$ constant it is possible to change the fundamental frequency. The equations given above are a good starting point for the antenna design. However for the best impedance matching, the coordinates of the feed point must be found through iterative simulations.

Modified bowtie type antennas have also been proposed fig.12, [33]-[34]. By the appropriate values of the geometrical parameters a bandwidth value that exceeds $90 \%$ can be obtained whereas the antenna exhibits small size.

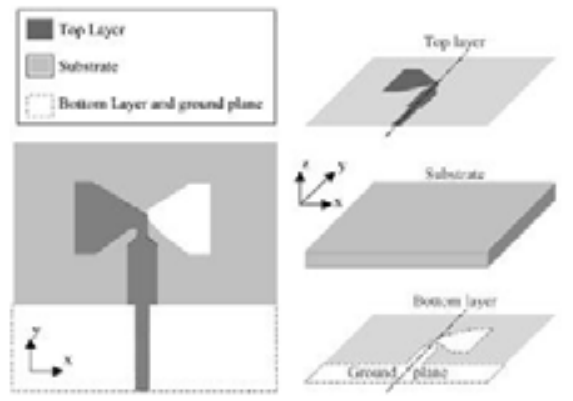

(a)

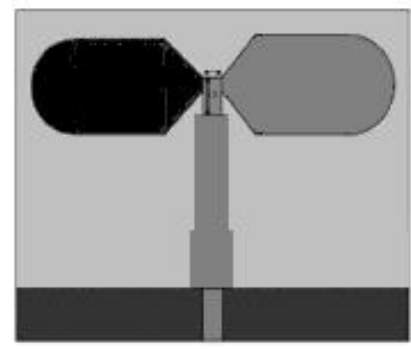

(b)

Fig. 12. Two configurations of the double sided wideband bow-tie antenna

The antenna of fig 12a consists of two identical printed bows, one on the top and one on the bottom of the substrate material. The top and the bottom bows are connected to the microstrip feedline and the ground plane through a stub and mitered transition to match the bow-tie with the selected characteristic impedance of the feedline.

By further modification of the bowtie antenna, namely by rounding of the bow patches, as shown in fig. 12b, wider bandwidth, higher co-polarization and lower cross-polarization for the UWB range can be achieved[34].

\subsection{Multiband microstrip antennas}

The rapid advances in the wireless communication industry demand novel antenna designs that could be used in more than one frequency bands and that will allow size reduction. For example mobile telephony's services require portable devices compatible with GSM900/DCS1800/UMTS2000 technology and the same equipment should also connect the users to WLAN networks based on 802.11 standards $(2.5 \mathrm{GH} / 5 \mathrm{GHz})$. So, the design of small antennas suitable for these devices is of great interest. Many techniques have been proposed for the design of radiating elements of this type, the great majority of which are microstrip antennas. The common characteristic, of almost the total, of the multiband printed elements is that they usually come from an initial patch of ordinary shape which in the following is perturbed. On the basis of the way of the shape perturbation, the multiband microstrip antennas would be classified in categories a) printed elements with incorporated slits or slots. [35]-[40] b) patches with more than one radiating elements conductively connected or/and inductively coupled[41] c) patches of specific shape as conductively connected cross dipoles[42], the bowtie[46], and the spiral [47]-[48] d) more than one stacked patches[49]. A separate category includes the fractal microstrip antennas which come from an initial simple printed element and are developed by a recursive process(\$3.5). 
At all the aforementioned categories the patches are produced starting from basic theoretical concepts and the procedures of development focus to similar targets. However, it has to be noticed that in no one of them a rigorous design process exists, namely a process which would lead the designer to begin from the same initial concept and, aiming at specific attributes of operation, to end in all cases in the same shape of patch. So, the design of a multi-frequency patch is substantially an art and the ways of texturing the patch' s surface as well as the techniques, used for optimization of the configuration, are just useful tools.

The designing of a multi-band printed antenna requires that the efficient operational features namely, gain greater than $0 \mathrm{~dB}$, almost uniform space distribution of the radiated power, if necessary, circular polarization and mainly small reflection coefficient at the feeding port must be ensured in all the frequency bands of operation. Furthermore all these properties must not be obtained at the cost of a complex feeding network, a non compact fabrication or an antenna arrangement of large size. Therefore the design of this type of antennas is a difficult task and beyond the used standard techniques any novel concept or modification of the existing methods would be useful and would lead to interesting antenna schemes.

\subsubsection{Slot loaded multiband microstrip antennas}

The technique of slot cutting the surface of the printed antenna, besides the broadening of the bandwidth has been proved effective in driving the patch to multi-frequency operation [35]-[40]. Various slot shapes have been proposed for the texturing of the patch; some indicative results are presented in figures 13 to 16 . Cutting a rectangular patch with Lshaped(Fig. 13a) or folded slit(fig. 13b) the antenna is considered to consist of two connected resonators of different sizes. The shorting pins at the corner of the patch permit to reduce its size, making this type of compact dual-band antenna suitable for applications in handset mobile communication units. The dimensions of the larger and the smaller sub-patches(Fig. 13a) can be designed to roughly resonate as quarter-wavelength structures at the prespecified frequencies. In the alternative configuration(Fig. 13b), the smaller sub-patch, that resonates at the higher frequency, starts from the feed point and extends into the center portion of the rectangular patch. In this way it is encircled by the slit and is surrounded by the outer larger sub-patch which resonates at the lower frequency.

Instead of using an L-shaped slit or a folded slit to obtain two separate sub-patches, an embedded U-slot can be used(Fig. 13c). In this configuration the smaller rectangular, of dimensions $\mathrm{L}_{2} \times \mathrm{W}_{2}$, resonates at the higher of the pre-specified frequencies and occupies the central portion of the original rectangular patch of dimensions $\mathrm{L}_{1} \times \mathrm{W}_{1}$ which resonates at the lower frequency. The lower $f_{L}$ and the upper $f_{H}$ operating frequencies of this design can be approximately determined from

$$
\begin{aligned}
& \mathrm{f}_{\mathrm{L}} \cong \frac{\mathrm{c}}{4\left(\mathrm{~L}_{1}+\mathrm{W}_{1}\right)} \\
& \mathrm{f}_{\mathrm{H}} \cong \frac{\mathrm{c}}{4\left(\mathrm{~L}_{2}+\mathrm{W}_{2}\right)}
\end{aligned}
$$

Where $\mathbf{c}$ is the speed of light in free space 


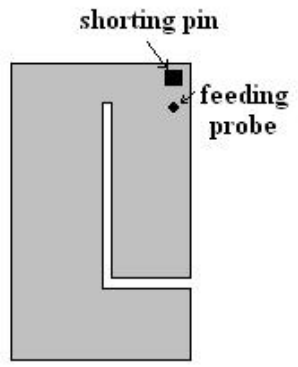

(a)

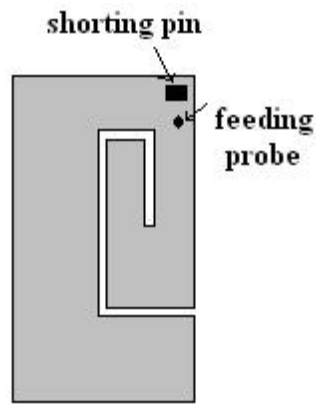

(b)

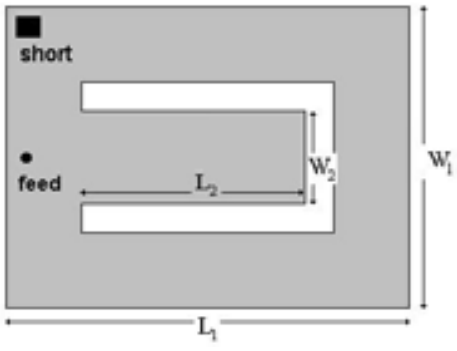

(c)

Fig. 13. Geometries of shorted rectangular patch antenna with a) an L-shaped slit b) a folded slit and c) U-slot, for dual frequency operation

In figures $14 \mathrm{a}$ and $14 \mathrm{~b}$ alternate types of perturbation of the patch's surface are shown[38]. The non radiating edges (with respect to the basic $\mathrm{TM}_{01}$ mode ) of the initial rectangular patch are modified by T-shaped notches in such a way that the current distribution of $\mathrm{TM}_{01}$ mode is similar to the current of the higher order $\mathrm{TM}_{21}$ mode (fig. 14b) driving the patch to similar operational characteristics at both frequencies. A further modification of the patch by two pairs of smaller T-notches with proper size results in triple frequency band operation(Fig.14c).

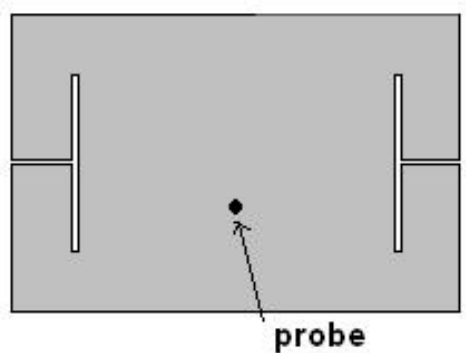

(a)

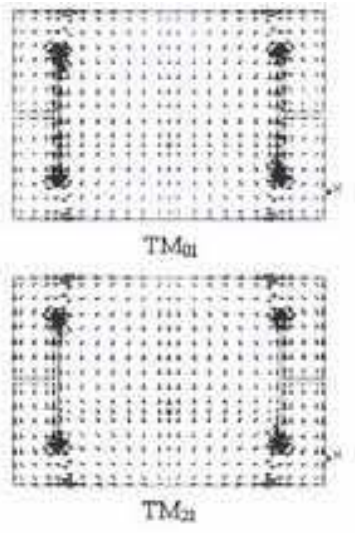

(b)

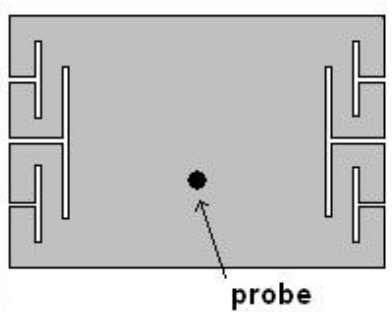

(c)

Fig. 14. (a), (c)Microstrip elements textured with single or multiple T-notches. (b) surface current density at $\mathrm{TM}_{01}$ and $\mathrm{TM}_{21}$ modes.

The concept of texturing the surface of the patch by U-slots that can effectively lead to a broadband operation can also be efficient in driving the element in multiband operation. Two U-slots [15], of different size (fig. 15a) or unequal slits combined with tiny circular slots (Fig15b)can ensure triple band operation[39].

Another configuration of microstrip antenna, loaded with slits is proposed in[40]. The patch (fig. 16a) has annular ring shape, is etched on a two layered dielectric substrate and is 


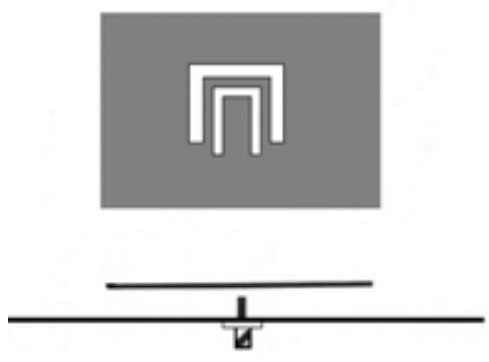

(a)

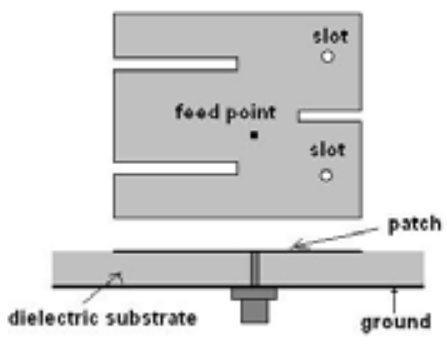

(b)

Fig. 15. Configurations of tri-band microstrip antennas a) dually U-slot loaded patch b)patch loaded with unequal slits and tiny circular slots

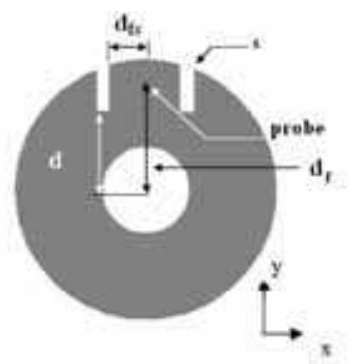

(a)

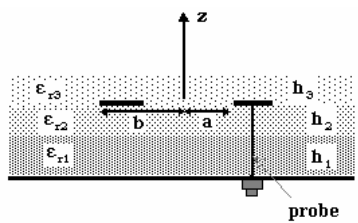

(b)

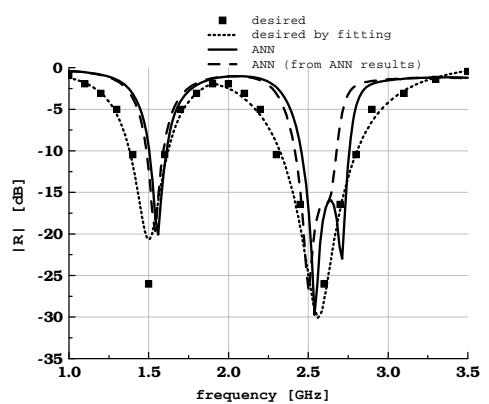

(c)

Fig. 16. (a), (b) Top and side view of a dual-band microstrip structure with multilayered substrate. (c) Reflection coefficient of the signal at the feeding point for a specific set of geometrical and material parameter values.

covered by a dielectric superstrate (fig. 16b). The inherent advantage of a ring antenna is, the property to resonate for a diameter less than $\lambda \mathrm{g} / 2(\lambda \mathrm{g}$ is the guiding wavelength of the equivalent linear microstrip line having width equal to that of the ring). This attribute means a physical size smaller than the size of the respective circular disc resonating at the same frequency. In the proposed configuration the total height of the substrate is high and ensures the broadness of frequency bands(fig. 16c). Additionally the slits in the vicinity of the position of the probe compensate efficiently the large inductive input impedance that inevitably comes from the thickness of the substrate. Due to the large number of the structural parameters of such an antenna an ordinary procedure, for example iterations with gradual variations of the values of the parameters, could be laborious and perhaps ineffective or unfeasible. So, a stochastic technique, which following an objective process, would yield a suitable antenna configuration.

The antenna of fig. 16a was designed using an Artificial Neural Network(ANN) algorithm(see $\S 4)$. The values of the structural parameters, as shown in figures $16 \mathrm{a}$ and $16 \mathrm{~b}$, were calculated by a properly designed and trained ANN at the input of which, the plot (sampled) of the scattering parameter of the signal at the feeding point was given. By this plot an antenna capable to resonate at $1.572 \mathrm{GHz}$ (GPS)and at $2.45 \mathrm{GHz}$ (WLAN based on protocol 802.11) was demanded from the ANN. The antenna implemented by the results of the output of the ANN, exhibits operation that meet these requirements, as shown in fig. 16c. 


\subsubsection{Multiple patches}

An alternative approach to the design of a multiband printed antenna is to use more than one elements with different size that resonate at different frequencies. An indicative structure [41] is shown in fig 17a. In detail, the antenna is an array of concentric microstrip annular rings printed on a double dielectric layer. The multi-frequency performance comes from the resonances of the individual rings and from the further modification made to the rings. The entire configuration has two inherent advantages a) each annular ring resonates having diameter less than $\lambda_{\mathrm{g}} / 2$, as explained for the antenna of fig 16a, b) a ring shaped printed element allows other rings of smaller radius, resonating at different frequencies, to be printed in the internal area, ensuring multi-frequency operation and at the same time compactness.

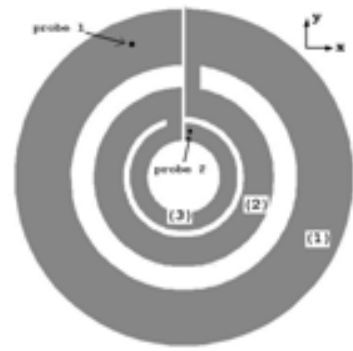

(a)

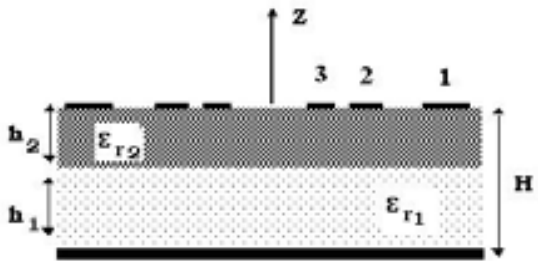

(b)

Fig. 17. Top and side-view of a multi-frequency microstrip antenna with three conductively connected rings

To find the frequencies of resonance of each ring it is necessary to solve the electromagnetic problem of printed annular ring antennas fed by probes. The basic and rigorous method for this solution, in spatial domain, is to determine the respective Green function [43]-[44]. The final form of this Green function, expanded in series of orthonormal eigenfunctions, is:

$$
\begin{aligned}
\mathrm{G}\left(\rho, \varphi \mid \rho^{\prime}, \varphi^{\prime}\right) & =\sum_{\mathrm{n}} \sum_{\mathrm{m}}\left[\sqrt{\frac{1}{\pi \mathrm{C}_{\mathrm{Jn}}}} \mathrm{J}_{\mathrm{n}}\left(\sqrt{\lambda_{\mathrm{mn}}} \rho\right)+\sqrt{\frac{1}{\pi \mathrm{C}_{\mathrm{Yn}}}} \mathrm{Y}_{\mathrm{n}}\left(\sqrt{\lambda_{\mathrm{mn}}} \rho\right)\right]\left[\sqrt{\frac{1}{\pi \mathrm{C}_{\mathrm{Jn}}}} \mathrm{J}_{\mathrm{n}}\left(\sqrt{\lambda_{\mathrm{mn}}} \rho\right)+\right. \\
& \left.+\sqrt{\frac{1}{\pi \mathrm{C}_{\mathrm{Yn}}}} \mathrm{Y}_{\mathrm{n}}\left(\sqrt{\lambda_{\mathrm{mn}}} \rho^{\prime}\right)\right] \cdot \frac{\cos (\mathrm{n} \varphi) \cos \left(\mathrm{n} \varphi^{\prime}\right)}{\lambda-\lambda_{\mathrm{mn}}}
\end{aligned}
$$

where $\rho^{\prime}, \varphi^{\prime}$ are the coordinates of the feeding probe, $a$ and $b$ are the inner and outer radius of the ring, $\lambda_{m n}$ is the $\mathrm{mn}^{\text {th }}$ eigenvalue, $\mathrm{J}_{\mathrm{n}}, \mathrm{Y}_{\mathrm{n}}$ are Bessel functions of the first and second kind respectively and $\mathrm{C}_{\mathrm{Jn}}$ and $\mathrm{C}_{\mathrm{Yn}}$ are the constants produced via the ortho-normalization process of the eigenfunctions

$$
\left.\left\{\begin{array}{c}
C_{J n} \\
C_{Y_{n}}
\end{array}\right\}=\frac{b^{2}}{2}\left[\begin{array}{c}
J_{n}{ }^{\prime} \\
Y_{n}{ }^{\prime}
\end{array}\right\}\left(\sqrt{\lambda_{m n}} b\right)\right]^{2}-\frac{a^{2}}{2}\left[\left\{\begin{array}{c}
J_{n}{ }^{\prime} \\
Y_{n}{ }^{\prime}
\end{array}\right\}\left(\sqrt{\lambda_{m n}} a\right)\right]^{2}
$$

In order the orthonormal eigenfunctions to satisfy the electromagnetic boundary conditions of the structure, the following equation must be satisfied 


$$
J_{n}^{\prime}\left(\sqrt{\lambda_{m n}} a\right) Y_{n}^{\prime}\left(\sqrt{\lambda_{m n}} b\right)+Y_{n}^{\prime}\left(\sqrt{\lambda_{m n}} a\right) J_{n}^{\prime}\left(\sqrt{\lambda_{m n}} b\right)=0
$$

Equation 16 is the characteristic equation of the problem and its solution gives the frequencies at which the ring resonates, that is

$$
\mathrm{f}_{\mathrm{mn}}=\frac{\sqrt{\lambda_{\mathrm{mn}}}}{2 \pi \sqrt{\mu \varepsilon}}
$$

The aforementioned formulation can be used to solve the inverse problem, namely to find the geometrical and material parameters of the ring antenna in order to resonate at the desired frequencies.

On the basis of the above theory, an antenna configuration with three rings was implemented in [41]. The rings were printed on the top side of a dielectric layer of $3.17 \mathrm{~mm}$ height $\left(\mathrm{h}_{2}\right)$ and dielectric constant 2.15(RT/Duroid 5880). The layer was suspended at a distance $4 \mathrm{~mm}$ over the metallic ground plane, namely $\varepsilon_{\mathrm{r} 1}=1$ and $\mathrm{h}_{1}=4 \mathrm{~mm}$. The frequencies of resonance of each one of the rings were calculated applying Eq. 17 and using as $\varepsilon_{\mathrm{r}}$ the effective dielectric constant of the corresponding suspended microstrip line[45]

$$
\sqrt{\varepsilon_{\mathrm{re}}}=\left[1+\frac{\mathrm{h}_{2}}{\mathrm{~h}_{1}}\left(\mathrm{c}_{1}-\mathrm{c}_{2} \ln \frac{\mathrm{w}}{\mathrm{h}_{1}}\right)\left(\frac{1}{\sqrt{\varepsilon_{\mathrm{r} 2}}}-1\right)\right]^{-1}
$$

where $\mathrm{c}_{1}=\left(0.8621-0.125 \ln \frac{\mathrm{h}_{2}}{\mathrm{~h}_{1}}\right)^{4} \quad, \quad \mathrm{c}_{2}=\left(0.4986-0.1397 \ln \frac{\mathrm{h}_{2}}{\mathrm{~h}_{1}}\right)^{4}$

The theoretically calculated frequencies of resonance are the frequencies at which each one of the rings would resonate without the presence of any other element. Therefore, they are just an approximate assessment of the expected resonances of the entire structure, due to the mutual coupling between the rings and the additional modification made to the construction. The modification was necessary because the input impedance of each ring was large and caused strong reflection of the feeding signal. In order to reduce the input impedance, narrow slits were created and the rings were conductively connected (Fig. 17a). As a consequence the entire printed structure has the shape of a spiral. The width of the slits and those of the printed interconnections of the rings, were found via repeated simulations, the object of which was the achievement of better feed matching. This type of antenna would also be designed using the techniques either of ANN or GA and PSO.

With respect to the feeding, two feeding probes were used and their positions were found with the criterion of feed matching. The employment of two probes was necessary because a single one could not excite all the theoretically predicted modes. Therefore at some frequency ranges (e.g. at $1.39-1.5 \mathrm{GHz}$ and at $2.82-2.83 \mathrm{GHz}$ ) both probes should be activated while at the other frequencies the antenna should operate via one of them.

In Fig.18 the measured and simulated scattering coefficients at the input of both probes are depicted. Due to the aforementioned reasons a difference between the theoretically predicted resonate frequencies and the measured ones, appears (see Table 1). Moreover, frequencies of operation smaller $1 \mathrm{GHz}$ exist. Their appearance is due to the fact that the path of the current along the spiral is larger than the perimeter of even the larger of the rings. These frequencies would not be predicted by the theoretical analysis, not even 

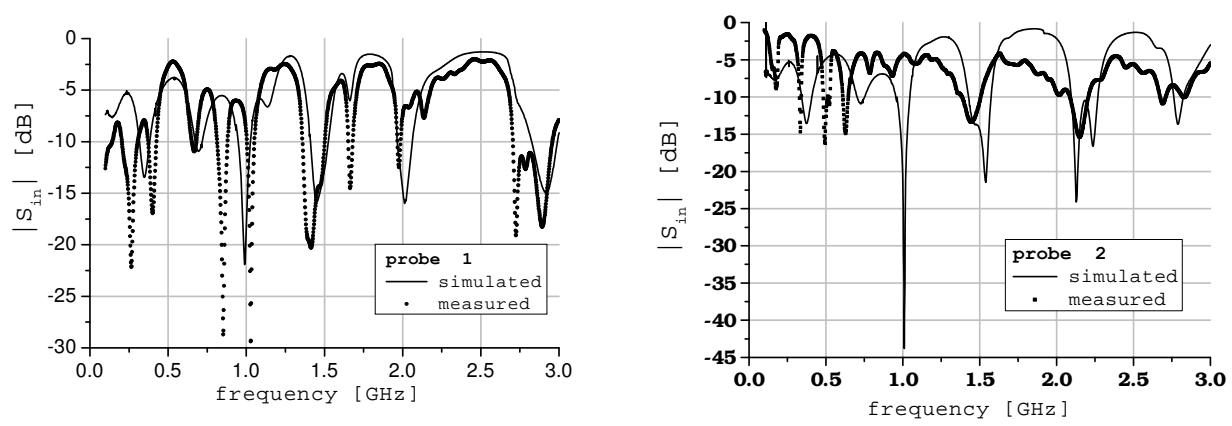

Fig. 18. Reflection coefficient of the signal at the feeding probes of the antenna shown in fig. 17

approximately, because in accordance to equations 16 and 17 the lower resonant frequency of all three rings is the basic resonant frequency of the larger one and it was found equal to $1.26 \mathrm{GHz}$, as shown in Table 1 . The results obtained via simulation approximate the respective measurements, in most of the frequency bands.

Table 1 includes the theoretical results for the frequencies of resonance and the respective ones found experimentally.

\begin{tabular}{|c|c|c|}
\hline \multicolumn{2}{|c|}{ Theoretical results } & \multirow{2}{*}{ Measured values [GHz] } \\
\hline Frequency[GHz] & mode & \\
\hline 1.26 & $\mathrm{n}=0 \quad \mathrm{~m}=1 \quad(\operatorname{ring} 1)$ & $\begin{array}{c}1.005-1.05 \text { (probe1) } \\
\text { (probe1) } \\
1.389-1.506 \text { (probe2) }\end{array}$ \\
\hline 1.7 & $\mathrm{n}=0 \quad \mathrm{~m}=1$ (ring 2$)$ & $1.653-1.682$ (probe2) \\
\hline 1.785 & $\mathrm{n}=0 \mathrm{~m}=2($ ring 1$)$ & 1.967-1.987 (probe1) \\
\hline 2.18 & $\begin{array}{ll}\mathrm{n}=0 & \mathrm{~m}=1(\operatorname{ring} 3) \\
\mathrm{n}=0 & \mathrm{~m}=3(\operatorname{ring} 1)\end{array}$ & 2.087-2.208 (probe2) \\
\hline 2.83 & $\mathrm{n}=0 \quad \mathrm{~m}=5$ (ring 1$)$ & (probe2) \\
\hline 2.94 & $\mathrm{n}=0 \quad \mathrm{~m}=3$ (ring 2) & (probe2) \\
\hline 3.06 & $\mathrm{n}=0 \quad \mathrm{~m}=2(\operatorname{ring} 3)$ & 2.7-2.975 (probe1) \\
\hline
\end{tabular}

Table 1.

Due to the annular ring shape of the elements of the antenna, the components of the radiated field do not differ by more than $5 \mathrm{~dB}$ on both main planes, in all frequency bands. Moreover the utilization of two probes offer the potential to increase the level of the gain applying a proper phase shift between the probes. Indicative results of the field pattern at $2 \mathrm{GHz}$ are presented in fig. 19.

\subsubsection{Bowtie patches loaded with slots}

The basic principle of creating slots on the surface of a printed antenna in order to drive it in multi-frequency operation can be applied to bowtie patches, as well. An indicative configuration(see Fig. 20) is proposed in [46]. The first step of this design is to choose the appropriate dimensions of the arms of the bowtie in order to ensure the first band of frequency. The calculations can be made either using equations 9 to 11 or equations for the design a triangular patch antenna. 


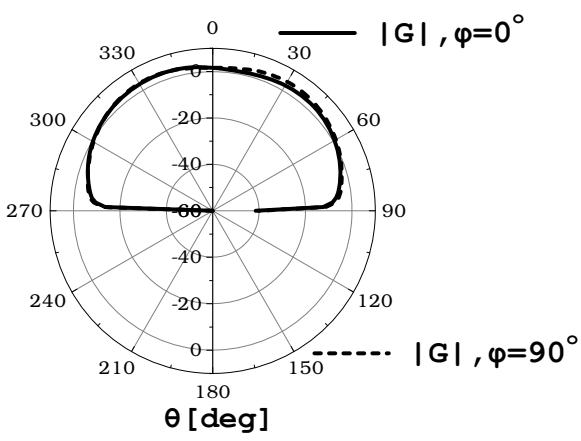

(a)

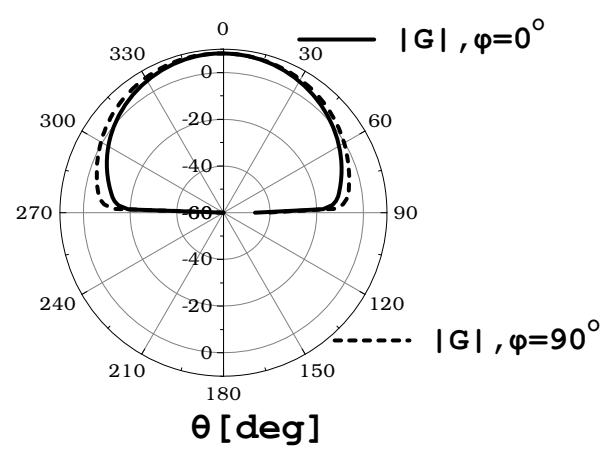

(b)

Fig. 19. Gain pattern at $2.0 \mathrm{GHz}$ of the antenna of fig. 17: a) probe 1 is fed, and b) probes are fed by $180^{\circ}$ out of phase.

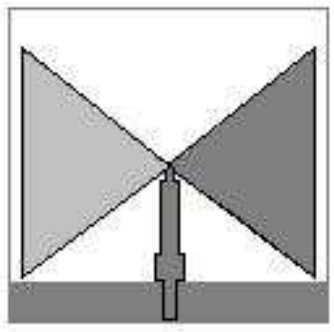

(a)

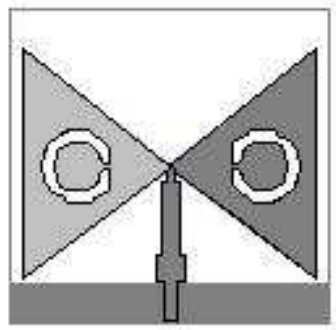

(c)

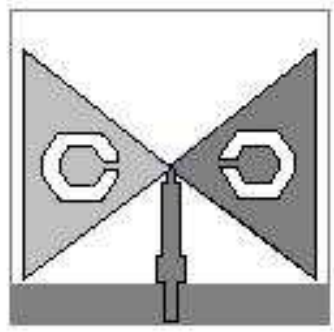

(b)

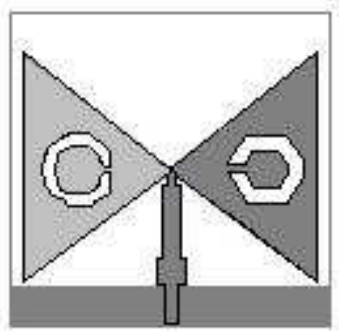

(d)

Fig. 20. Bowtie microstrip antennas: a) the ordinary configuration, (b),(c),(d)modified patches, by loading with slots of various shapes, for multiband operation.

The resonant frequency corresponding to the various modes $\mathrm{TM}_{\mathrm{mn}}$ of the equilateral triangular microstrip antenna is [45]

$$
\mathrm{f}_{\mathrm{r}}=\frac{2 \mathrm{c}}{3 \alpha \sqrt{\varepsilon_{\mathrm{r}}}} \sqrt{\mathrm{m}^{2}+\mathrm{mn}+\mathrm{n}^{2}}
$$

Where $c$ is the velocity of light in free space, $\alpha$ is the side length of the bow-tie patch and $\varepsilon_{\mathrm{r}}$ is the dielectric constant of the substrate. 
The above equation is valid when the triangular resonator is enclosed by a perfect magnetic wall. In the case it is not valid the replacement of side length $\alpha$ by an effective value $\alpha_{\mathrm{e}}$ has been suggested. An approximate expression for $\alpha_{\mathrm{e}}$ produced by curve fitting experimental and theoretical results for the resonant frequency for $\mathrm{TM}_{10}$ mode is given [45], by

$$
\alpha_{\mathrm{e}}=\alpha\left[1+2.199 \frac{\mathrm{h}}{\alpha}-12.853 \frac{\mathrm{h}}{\alpha \sqrt{\varepsilon_{\mathrm{r}}}}+16.436 \frac{\mathrm{h}}{\alpha \varepsilon_{\mathrm{r}}}+6.182\left(\frac{\mathrm{h}}{\alpha}\right)^{2}-9.802 \frac{1}{\sqrt{\varepsilon_{\mathrm{r}}}}\left(\frac{\mathrm{h}}{\alpha}\right)^{2}\right]
$$

where $\mathrm{h}$ is the thickness of the substrate.

In the second step of the design process circular or six sided polygon slots are incorporated to the bow patches. This texturing drives the antenna to dual-frequency operation: the initial frequency, obtained by the first step of design, and a second desired one that can be obtained by properly choosing the size of the slots (Figs. 20b and 20c). A tri-band performance can be achieved cutting, in the two parts of the antenna, slots either of different size or of different shape, as shown in Fig. 20d.

In all configurations implemented in [46], the printed elements are fabricated one on the bottom and the other on the top side of the dielectric substrate. The feeding method depends on the selected bow-tie configuration and the parameter values of the feeding network are calculated by the high frequency transmission microstrip line theory

\subsubsection{Spiral antennas}

Multi-frequency operation can also be achieved using spiral printed antennas. A suitable modification of the ordinary shapes of the printed spirals, especially of the rectangularly shaped, has been proved to be effective to multi-band performance. Three indicative proposed structures are shown in figure 21. The design of all three is based on a modified spiral line technique model and on the use of shorting -pin method[47]-[48]. An advantage of these antennas is that they have the ability to resonate in frequencies much smaller than the other multiband anennas can, having at the same time very small size. The key for this performance is the increased length of the current path along the conductor area due to the spiral shape of this conductor. For the structures of figures $21 \mathrm{a}$ and $21 \mathrm{~b}$, two ways of folding the conductor in order to maximize its length were used. One is the standard rectangular folding and the other is the folding the conductor back onto itself in the two dimensions. Each configuration has three dependent variables for a constant antenna area, namely the number of bends in the conductor, the conductor width and the gap between the conductors. Attention has to be drawn to the thickness of the conductor because it has a lower limit, set by the diameter of the probe and the shorting pins. Using these configurations, in [47], dual band operation was obtained at frequencies being both smaller than $1 \mathrm{GHz}$.

A multi band operation can also be obtained by the configuration of fig. 21c. In this case a small number of bends for the spiral shape are used and the corners are truncated to allow smooth current flow. The frequencies of resonance and the corresponding bandwidths as well as the matching, are controlled by the line's width and length, the gaps between the lines, the position of the shorting pin and the feed point. The utilization of a dual slab substrate, the lower slab being of air or foam, may enhance the bandwidth. This spiral configuration due to the relatively small length of the conductor is recommended for high frequency applications. In [48] by proper selection of the parameter values a dual band operation was achieved. One from $2.37 \mathrm{GHz}$ to $2.47 \mathrm{GHz}$ and another, of large width, from $5 \mathrm{GHz}$ to $6.3 \mathrm{GHz}$. 


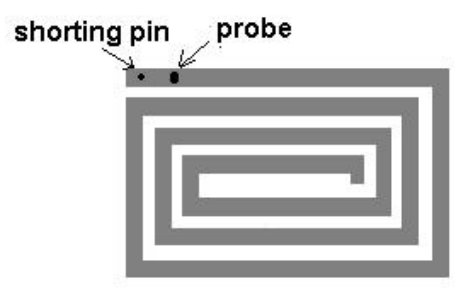

(a)

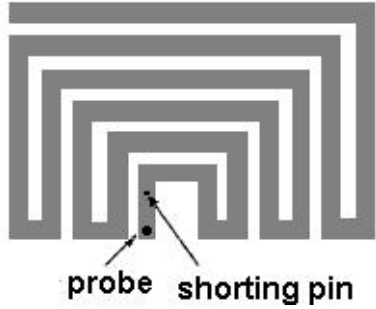

(b)

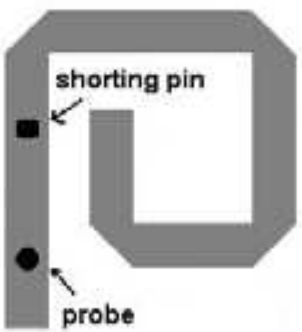

(c)

Fig. 21. Microstrip spiral configurations a) the ordinary shape b)the conductor is folded back onto itself c) small number of bends and truncated corners.

\subsubsection{Fractal antennas}

Fractal technique has been applied to many areas of science including fractal electrodynamics in which fractal concepts are combined with electromagnetic theory for the purpose of investigating a new class of radiation, propagation and scattering problems. Thus, fractal antennas are an innovative type of radiating elements coming from the inspired combination of these two theories.

The fractal antenna engineering focuses in two promising research areas : the first deals with the analysis and design of fractal radiating elements and the second, concerns to the application of the fractal theory to the design of antenna arrays[50]-[54]. Both antenna types have attributes, highly desirable in military as well as in commercial sectors. The majority of fractal antenna elements, are printed configurations, have compact size, low profile and cost, multi-band operation, easy feeding and, potentially, their operation could be optimized by suitable modification of their shape. On the other hand the application of the fractal technique to the design of an antenna array can produce radiating systems of large size, and as a consequence of high gain, with frequency-independent or multi-band operation and radiation patterns with low side-lobe level. Moreover, the elements of the array can be fed one by one, thus operating as Direct Radiating Antennas(DRAs) and can function as phased arrays[55]-[57].

Fractal objects can be classified in two categories: deterministic and random. Deterministic are those that are generated of several scaled-and rotated copies of themselves using a recursive algorithm. Random fractals also contain elements of randomness that allow simulation of natural phenomena. Procedures and algorithms for generating fractals both deterministic and random have been found. However most of the proposed fractal antennas have been designed with a deterministic fractal process.

The fractal technique is based on the idea of realization the operational characteristics of the antenna by repeating an initial radiating structure in arbitrary or regular scales. The self similarity properties of the produced antenna configurations are translated into their electromagnetic behavior and since the initial antenna was introduced, the higher stages of fractaly developed geometries would have, for example, the attribute of multi-frequency operation. The basic scheme of a fractaly designed antenna is a generating, termed also initiating, radiating element. In particular, the entire antenna can be formed recursively through repetitive application of the generating element under a specified scaling factor which is one of the parameters of the problem. This process is realized following potentially 
two different strategies: By one of them, the repetition of the generating antenna is made in such a way that its entire size gets larger from stage to stage of fractal building. By the second strategy, the entire area, which the final antenna is permitted to occupy, is defined a priori. Then, by the process of the proper repetition of the generator, the available area is filled by scaled replicas of the generator.

This space filling leads to antenna configurations with electrically large lengths although they have small size thus, constituting substantially a miniaturization technique producing elements efficiently packed into small areas, therefore suitable for installation in portable telecommunication devices.

The theoretical analysis as well as the interpretation of the operation of a microstrip fractal antenna is based on the underlying relation between a fractal resonator and a microstrip antenna. The physical problem of the operation of fractal resonators is often referred to, in the literature, as the determination of the 'fractal drum' vibration modes. The simplest example of a surface fractal resonator is the fractal drum, where a vibrating membrane is bounded by a fractal curve, its vibration modes named 'fractions'. The vibration modes of fractal drums exhibit some interesting properties such as the existence of localized modes. In these modes the vibration is strongly localized in certain parts of the membrane. These vibrational states are obtained after the solution of the Helmholtz equation with the appropriate boundary condition. For surface fractals, a distinction is made between Neumann and Dirichlet fractions according to the boundary condition that is applied.

On the other hand microstrip antennas can be modeled, in a first approximation, as a cavity. For a microstrip patch antenna of electrically small height of substrate, the field distribution can be found with very good accuracy from the eigenfuntions of the Helmholtz equation subject to the Neumann boundary condition. Therefore, it is expected that fractal boundary microstrip antennas will exhibit vibration modes similar to those ones of the fractal drum.

The most popular fractal geometries, referred in the literature, which were proved efficient in antenna design are the Koch fractal, the Sierpinski gasket or carpet fractal, the Hilbert fractal, the Minkowski and the Square Curve fractals. All of them were proved to yield printed antennas, planar or conformal, with multi-frequency performance as well as easy feeding, satisfactory polarization properties and gain, whereas they have small size. All these attributes make them an attractive choice for portable telecommunication equipment.

\subsection{5 a) Koch: direct and inverse fractal islands}

The Koch fractal microstrip patches are commonly used in virtue of their attractive properties: they have small size, a single feeding port is enough and their higher order modes result in directive radiation patterns[58]-[60]. The basic geometry of the Koch island is obtained by replacing the sides of an equilateral triangle by a Koch curve. The triangle is the generator and the higher stages of fractal development follow the respective stages of the Koch fractal curve, as shown in fig. 22.

The Koch fractal curve is constructed as follows: A straight line of length a, is the generator and is first divided into three equal segments. The middle segment is removed and replaced by two segments having the same length to generate an equilateral triangle. This 4-sides line is the first fractal stage, its direct length from one end to the other is equal the length of the generator but the summation of the lengths of all four segments is greater and is calculated by the general expression $L_{n}=\alpha\left(\frac{4}{3}\right)^{n}$, where $\mathrm{n}$ is the number of iteration. It is noticed 
that to use the Koch curve in order to build the Koch island, the length of the generator should be equal to the side of the triangle. In the next stage the process is repeated for the 4 segments generated at the first iteration, leading to the drawing of fig. 22c for the curve, and the $22 \mathrm{~g}$ for the island. The process in the next steps is evident.

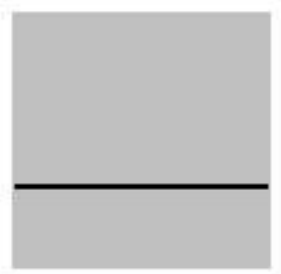

(a) generator

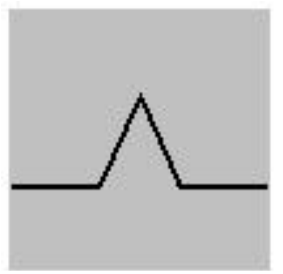

(b) $1^{\text {st }}$ stage

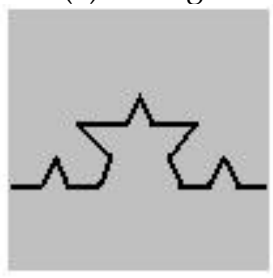

(c) 2nd stage

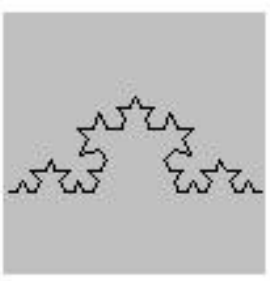

(d) $3^{\text {rd }}$ stage

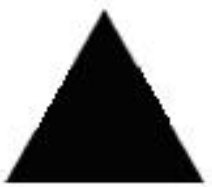

(e) generator

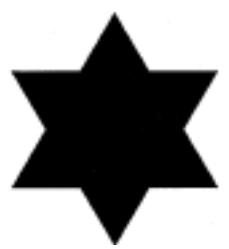

(f) $1^{\text {st }}$ stage

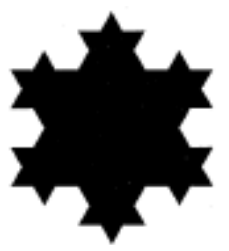

(g) $2^{\text {nd }}$ stage

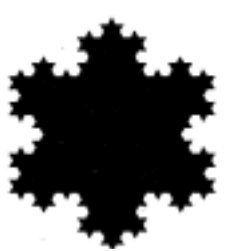

(h) $3^{\text {rd }}$ stage

Fig. 22. (a)-(d) The generator and the first three stages of the Koch fractal curve. (e)-(h) The generator and the first three stages of the Koch fractal island and potentially printed antenna.

A basic advantage of the Koch patch, as mentioned above, is its attribute to resonate efficiently at frequencies lower than those of a regular patch of equal size. It has been verified via electromagnetic simulation as well as experimentally. To estimate the superiority of the Koch fractal to a conventional triangular patch a comparison between their sizes is necessary. At each new iteration $n$ the area of the Koch island increases. Let $A_{n}$ be the area at $n^{\text {th }}$ iteration, then the area of the next iteration can be computed as 


$$
A_{n+1}=A_{n}+\frac{\sqrt{3}}{12}\left(\frac{4}{9}\right)^{n-1} \alpha^{2}
$$

Where $a$ is the side of the initial triangle that has an area

$$
A_{0}=\frac{\sqrt{3}}{4} \alpha^{2}
$$

The geometry series given by (21) converges to

$$
\mathrm{A}=\frac{2}{5} \sqrt{3} \alpha^{2}
$$

All the iterations are circumscribed inside a circumference of radius $r=\sqrt{3} \alpha / 3$. On the other hand the perimeter increases at each new iteration. The overall perimeter for iteration $\mathrm{k}$ is given by $1_{\mathrm{n}}=3 \alpha\left(\frac{4}{3}\right)^{\mathrm{n}}$.

This evolution process leads to a fractal object with an infinite perimeter bounding a finite area. Despite of the increasing irregularity of the boundary, the manufacturing process does not become more complex at each new iteration. The patch can be manufactured by standard photo-etching techniques. The fundamental limitation in building the antenna is given by the resolution of the photo-etching process. When the number of iterations is increased the new added details in the structure cannot be resolved and they are not reproduced in the manufactured element.

In fig 23 the frequency dependence of the input impedance of microstrip Koch atennas for first five iterations are presented. All cases concern to the fundamental mode and the results were received by numerical analysis with the Method of Moments(MoM)[58].
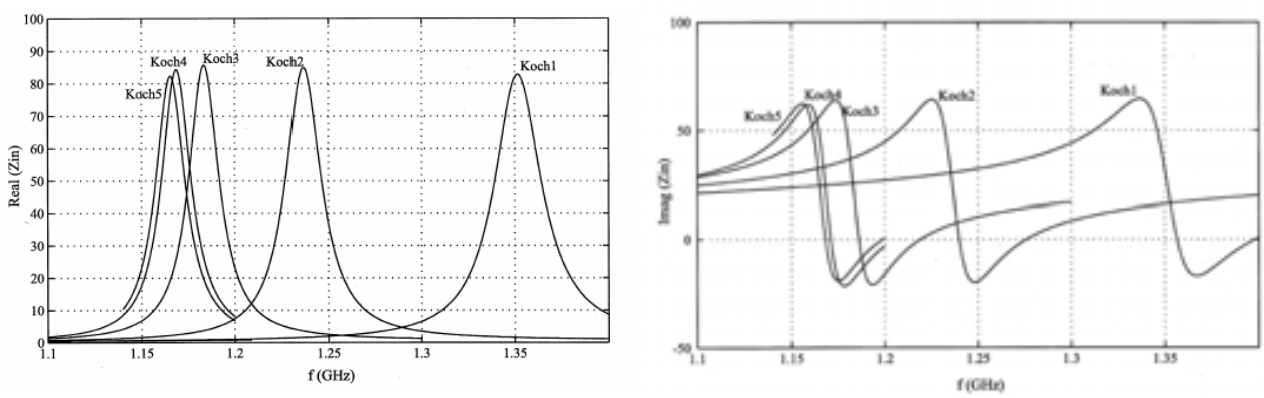

Fig. 23. Indicative results of the input resistance and reactance of a Koch fractal microstrip patch for several fractal iterations

The Koch patches were generated by an equilateral triangle whose side was $118.2 \mathrm{~mm}$. They were printed on dielectric substrate with dielectric constant $\varepsilon_{\mathrm{r}}=4.17$ and thickness $\mathrm{h}=1.6 \mathrm{~mm}$. The printed metallic patch was placed $3.4 \mathrm{~mm}$ from the ground plane. So, a dual layer substrate was created. The patches were fed by a coaxial probe and the feed point was at the same location for all the patches and was placed at $15.5 \mathrm{~mm}$ from the center of the patch. An 
interesting conclusion can be derived from the input impedance plot. The fundamental resonant frequency decreases when the number of iterations increases. Nevertheless this reduction tends to limit by the increment of iterations. So, the difference between the resonant frequencies of stage 4 and stage 5 is very small. This performance proves the great attribute of this type of antenna to resonate at small frequencies having at the same time small size. For example the generator, namely the ordinary equilateral triangle, of the above structure resonates approximately at $2.2 \mathrm{GHz}$. It can be verified if we use eq. (19) and set as $\varepsilon_{\mathrm{r}}$ for the dual layer substrate an equivalent value resulting from the equation $\varepsilon_{\mathrm{r}} \approx \sum_{\mathrm{i}=1}^{2} \mathrm{~h}_{\mathrm{i}} / \sum_{\mathrm{i}=1}^{2} \frac{\mathrm{h}_{\mathrm{i}}}{\varepsilon_{\mathrm{ri}}}$. So, the ratio of $2.2 \mathrm{GHz}$ over the $\sim 1.15 \mathrm{GHz}$ at which resonates the Koch island of the $5^{\text {th }}$ stage is about 1.9. At the same time, in accordance to equations (21) and (22) the ratio $\frac{\text { Area of the } 5^{\text {th }} \text { stage Koch island }}{\text { Area of the triangle }} \simeq 1.6$. If , instead of using the $5^{\text {th }}$ stage Koch island, an ordinary equilateral triangular patch was used, the length of its side had to be 1.9 times the side of the initial triangle in order to resonate at $\sim 1.15 \mathrm{GHz}$. In this case the ratio of the surfaces of the two triangles had to be equal to $1.9^{2}=3.61$ instead of 1.6.

It is worthwhile to note that the concept Koch fractal can be used to build monopole antennas, enlongated or loop formed, which exhibit multi-frequency operation. In [59] a Koch loop monopole is proposed(fig. 24). It was designed to resonate at the bands of $2.45 \mathrm{GHz}$ and $5.25 \mathrm{GHz}$ therefore capable to serve a $802.11 \mathrm{~b} / \mathrm{g}$ WLAN system.

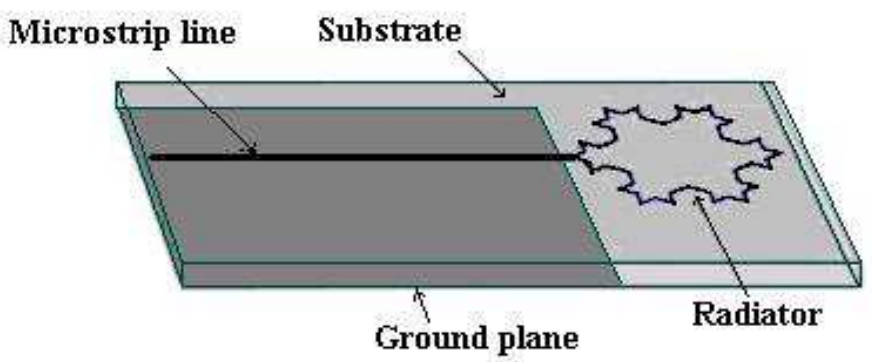

Fig. 24. The geometry of a planar monopole antenna with Koch fractal loop.

An alternative design of a fractal planar object, based on the Koch concept is shown in figure 25a. In this case, the generator is a square patch. The first stage of the fractal building can be produced by dividing each side of the initial square element into three equal segments. Then the middle segment of each size was removed and replaced by two segments of equal length and generating an equilateral triangle. Up to this point the process is similar to that of the Koch curve. In the following this triangle was directed inward to the initial square patch and was subtracted from it.

This configuration has been proposed in [60] and was successfully used to build a tri-band microstrip antenna configuration for GPS and terrestrial radio services. The initial square patch had side length equal to $78.25 \mathrm{~mm}$ and was printed on a dielectric substrate having $\varepsilon_{\mathrm{r}}=1.046$ and height $6 \mathrm{~mm}$. The patch of the first stage fed solely by one probe had small gain and single band operation. The target was the antenna to operate in three frequency ranges 
(GPS, DCS1800 and 2.7GHz). The desired performance was obtained by the configuration shown in Fig. 25b. It came from the combination of four patches of the first stage, lightly overlapped. Two probes were used and the operation was enhanced by two pins. The suitable position of the probes and the pins was found by a number of simulations whereas the relatively large size of the entire printed scheme, due to the four fractal patch , ensured the satisfactory high gain. For best performance, the presence of the probes and pins was exploited. A phase difference between the feeding probes was inserted and on-off switching of the pins, depending on frequency area, was imposed. These situations are shown in Table 2.

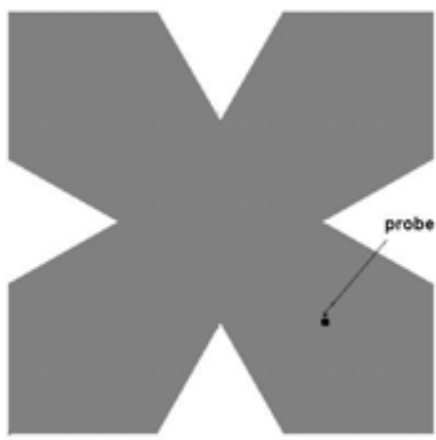

(a)

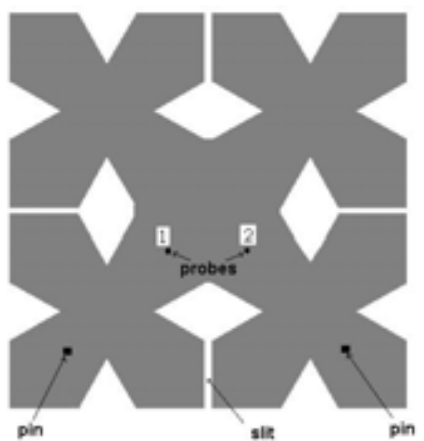

(b)

Fig. 25. Microstrip patch antenna for GPS and terrestrial radio services: a) A square patch modified via the first stage inverse van Koch fractal curve b) The compact radiating structure produced by the union of four fractal elements.

\begin{tabular}{|c|c|c|c|}
\hline & $\begin{array}{c}\text { GPS } \\
(1.575 \mathrm{GHz})\end{array}$ & $\begin{array}{c}\text { DCS } \\
1.78 \mathrm{GHz}-1.9 \mathrm{GHz}\end{array}$ & $2.67 \mathrm{GHz}-2.78 \mathrm{GHz}$ \\
\hline Current of probe 1 & 1 & 1 & 1 \\
\hline Current of probe 2 & $1 \mathrm{e}^{\mathrm{j} \pi / 2}$ & 1 & $1 \mathbf{e}^{\mathrm{j} \pi / 2}$ \\
\hline pins & off & switched to ground & switched to ground \\
\hline
\end{tabular}

Table 2.

The results for the scattering coefficient of the signal at the feed input are depicted in figure 26 and show the tri-band operation.

The gain patterns of the antenna are illustrated in figures 27 and 28. At $1.575 \mathrm{GHz}$ (fig. 27), the system appears a broadside radiation suitable for a GPS link when the antenna is horizontally positioned and the total maximum gain is $6.8 \mathrm{~dB}$.

The distribution of the radiated power in the DCS frequency range is shown in fig. 28. In this case the field is linearly polarized on yz-plane having a maximum gain value $4.9 \mathrm{~dB}$, approximately constant in a range $\pm 40^{\circ}$ about the broadside direction. In xz-plane it is also linearly polarized and the $\mathrm{G}_{\theta}$ component has maximum value $(4 \mathrm{~dB})$ at the broadside and minimum(-23dB) at $\pm 36^{\circ}$ apart from the broadside whereas the $G_{\varphi}$ component has maximum $(4 \mathrm{~dB})$ at a direction $30^{\circ}$ apart from the broadside). 

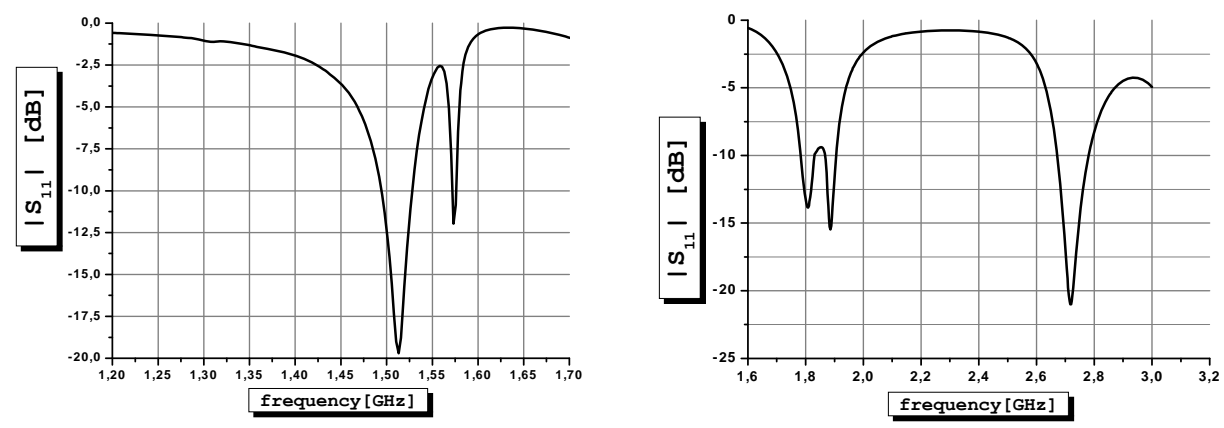

Fig. 26. Operation of the antenna of fig. $25 \mathrm{~b}$ a) Scattering coefficient at the input of each probe in the range1.2GHz to1.7GHz and b) Scattering coefficient at the input of each probe in the range $1.6 \mathrm{GHz}$ to $3 \mathrm{GHz}$

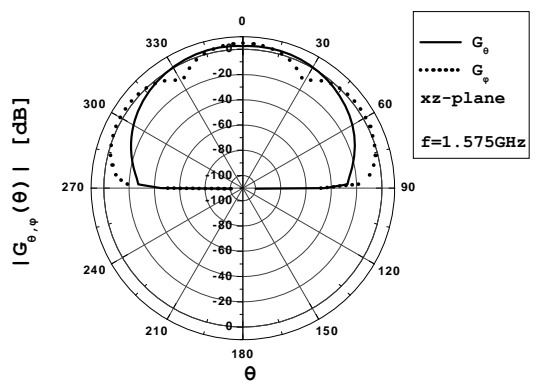

(a)

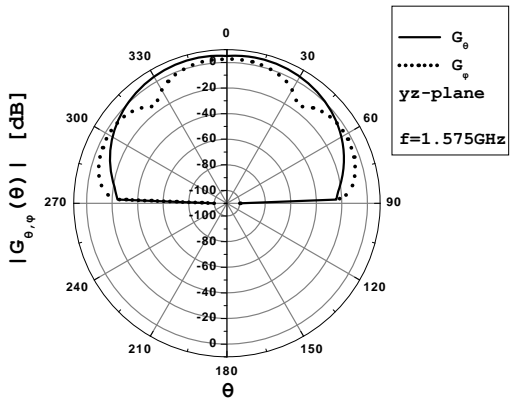

(b)

Fig. 27. Radiation patterns of the antenna of fig. 25 : the power gain components at $1.575 \mathrm{GHz}$ on $\mathrm{xz}$ - and yz-plane
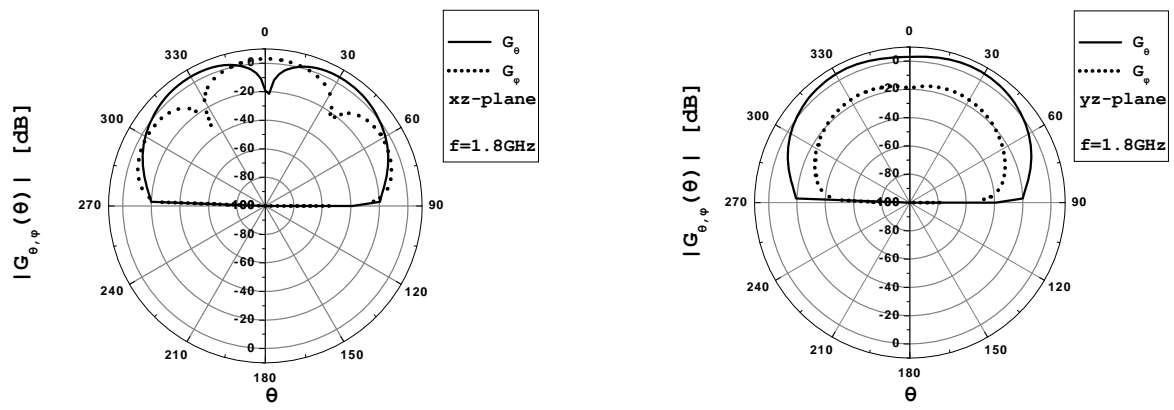

Fig. 28. Radiation patterns of the antenna of fig. 25 : the power gain components at $1.8 \mathrm{GHz}$ on $x z-$ and yz-plane 


\subsection{5 b) Sierpinski fractals}

Another fractal concept widely used for the design microstrip antennas is the Sierpinski fractal[61]-[69]. Various Sierpinski fractal objects have been proposed: The Sierpinski Gasket(or Triangle), the Sierpinski Carpet ( or rectangle), the Sierpinski Pentagon and the Sierpinski Hexagon. Judging from the literature the most efficient shapes for antenna applications are the carpet and especially the gasket. Monopole or dipole gasket fractal microstrip schemes have been proposed as multifrequency antennas.

Although the Sierpinski objects are based on different geometrical basis, they share the same construction principle. The geometrical construction of the popular Sierpinski gasket begins with an equilateral triangle which is considered as generator(fig. 29a). The next step in the construction process is to remove the central triangle, namely the one with vertices that are located at the midpoints of the sides of the original triangle. After the substruction, three equal triangles remain on the structure, each one being half of the size of the original one(fig. 29b). This process is then repeated for the three remaining triangles etc(figures 29c, $29 \mathrm{~d}$ ). If the iteration is carried out an infinite number of times the ideal fractal Sierpinski gasket is obtained. In each stage of the fractal building each one of the three main parts of the produced structure is exactly similar to the whole object, but scaled by a factor. Thus the Sierpinski gasket, as well as the other Sierpinski objects, are characteristic examples of self similar schemes.

It has to be pointed out that from an antenna engineering point of view the black triangular areas represent a metallic conductor whereas the white triangular represent regions where metal has been removed.

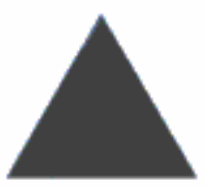

generator

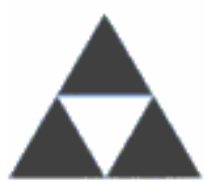

$1^{\text {st }}$ order

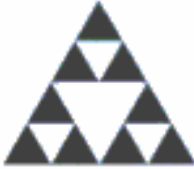

2nd order

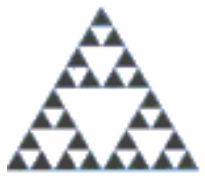

3rd order

Fig. 29. The generator and the first three stages of the Sierpinski fractal gasket.

Figure 30 shows a Sierpinski gasket monopole printed antenna. Typically such antennas exhibit a log-periodic spacing of resonant frequencies as well as an increase in the impedance bandwidth at higher bands. It is interesting to note that the band number $\mathrm{n}$ and the iteration $\mathrm{k}$ are interchangeable. For example the band zero and the $0^{\text {th }}$ iteration correspond to the fundamental resonance of the antenna. The first band and the first fractal iteration correspond to the first log-periodic resonant frequency. Therefore after the first fractal iteration two resonant frequencies are available : the fundamental and the first log periodic frequency. This is valid for other higher fractal iterations.

The specific positions of the frequency bands depend on the geometry of the generator and the parameter values of the dielectric substrate. It has to be noticed that the generator would potentially be not an equilateral triangle, namely the angle(flare) that corresponds to the vertex at which the feeding is applied would be not equal to $60^{\circ}$. Many such configurations have been proposed. The potential to select another value for this angle is an advantage because there are two geometrical parameters to control the frequencies of resonance. The height of the triangle and the flare angle. Indicative configurations are shown in fig.31a, and the respective input impedance diagrams are depicted in fig. 31b [64]. 


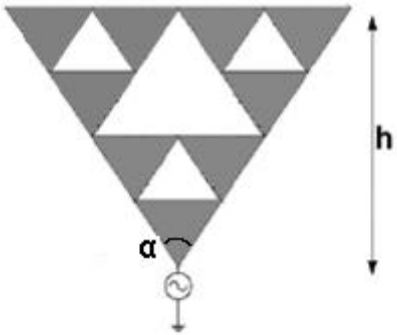

(a)

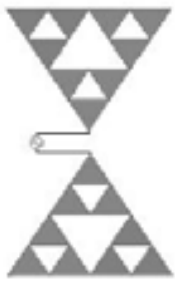

(b)

Fig. 30. The Sierpinski monopole(a) and dipole(b) printed antenna

From the design point of view, mathematical expressions for the calculation of the frequencies of resonance of the Sierpinki gasket, are necessary. The most recent available formula[65] in terms of the structural parameters and the order of iteration, for a monopole(fig. 30a) with flare angle equal to 60 o is that of eq. (23). This expression includes the parameters of the geometry of the gasket as well as the thickness and the dielectric constant of the substrate.

$$
\mathrm{f}_{\mathrm{r}} \cong \mid \begin{aligned}
& (0.15345+0.34 \rho x) \frac{\mathrm{c}}{\mathrm{h}_{\mathrm{e}}}\left(\xi^{-1}\right)^{\mathrm{n}} \text { for } \mathrm{n}=0 \\
& 0.26 \frac{\mathrm{c}}{\mathrm{h}_{\mathrm{e}}} \delta^{\mathrm{n}} \quad \text { for } \mathrm{n}>0
\end{aligned}
$$

Where $\xi=\frac{h_{n}}{h_{n+1}}$ is the ratio of the height of the gasket in the $n^{\text {th }}$ iteration to that in the $(\mathrm{n}+1)^{\mathrm{th}}$ iteration, $\delta=1 / \xi$ is the scale factor $\rho=\xi-0.230735$ and $\mathrm{x}=\mid \begin{aligned} & 0, \mathrm{n}=0 \\ & 1, \mathrm{n}>0\end{aligned}$.

Moreover

$$
\begin{gathered}
\mathrm{h}_{\mathrm{e}}=\frac{\mathrm{s}_{\mathrm{e}} \sqrt{3}}{2} \\
\mathrm{~s}_{\mathrm{e}}=\mathrm{s}+\mathrm{t}\left(\varepsilon_{\mathrm{r}}\right)^{-0.5}
\end{gathered}
$$

where $\mathrm{s}$ is the length of the side of the gasket and $\mathrm{t}$ and $\varepsilon_{\mathrm{r}}$ are the thickness and the dielectric constant of the substrate. The above equation is valid even in cases where the geometry is perturbed to get different scale factors

In practice the given parameter value is the frequency of resonance and the values of $t$ and $\varepsilon_{\mathrm{r}}$ are selected by the designer. So, for a specific value of $n$, the required parameters are those of the geometry of the gasket. For these calculations the side length of the generating triangle of the gasket is given by the expression

$$
\mathrm{S} \cong \mid \begin{aligned}
& \frac{1}{\sqrt{3}}(0.3069+0.68 \rho x) \frac{\mathrm{c}}{\mathrm{f}_{\mathrm{r}}}\left(\xi^{-1}\right)^{\mathrm{n}}-\frac{\mathrm{t}}{\sqrt{\varepsilon_{\mathrm{r}}}} \text { for } \mathrm{n}=0 \\
& \frac{0.52}{\sqrt{3}} \frac{\mathrm{c}}{\mathrm{f}_{\mathrm{r}}} \delta^{\mathrm{n}}-\frac{\mathrm{t}}{\sqrt{\varepsilon_{\mathrm{r}}}} \quad \text { for } \mathrm{n}>0
\end{aligned}
$$




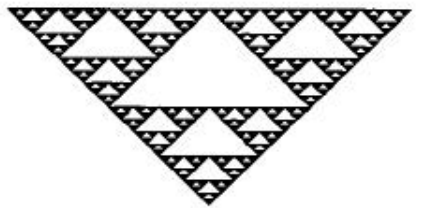

$a=90 \mathrm{deg}$

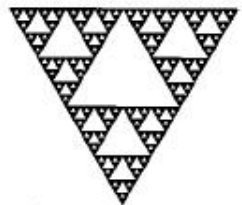

$a=60 \mathrm{deg}$

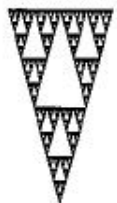

$a=30 \mathrm{deg}$

(a)

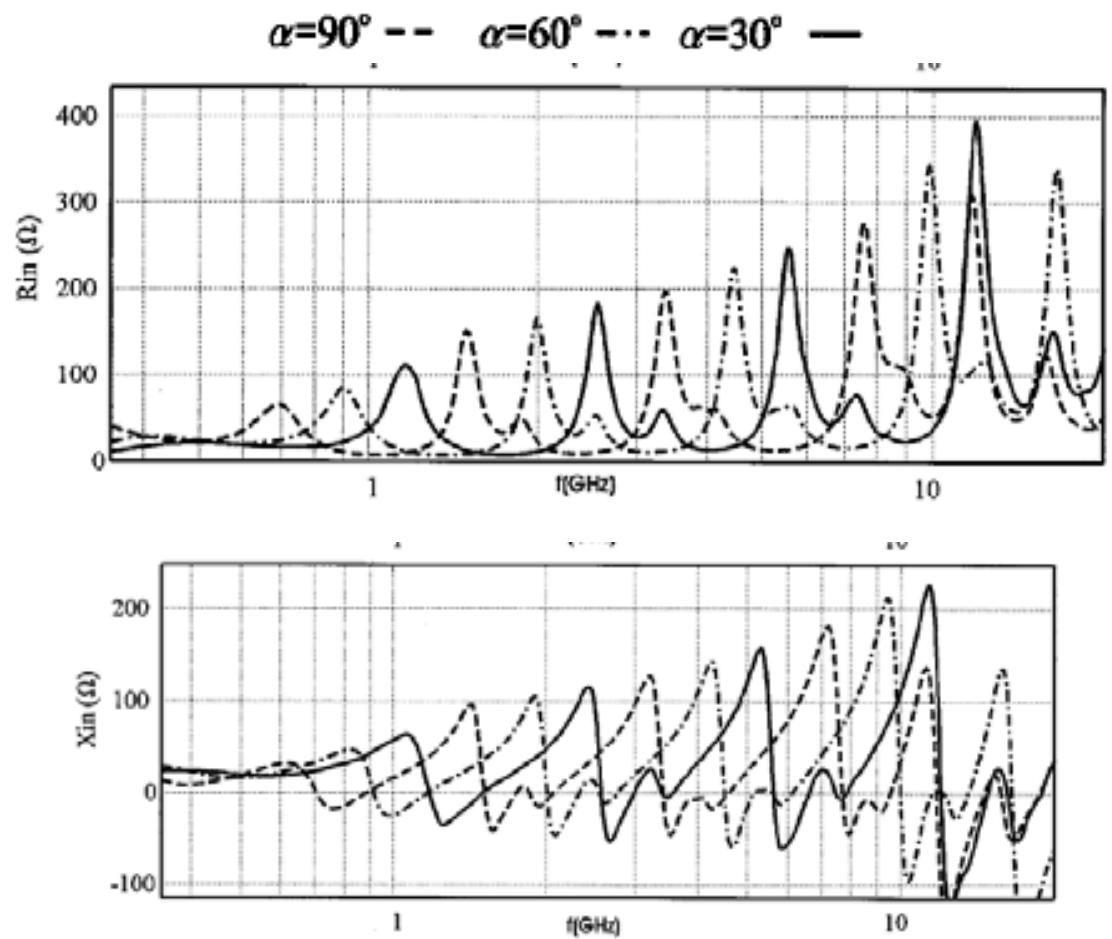

(b)

Fig. 31. a)Sierpinski gasket antennas with different flare angle b) Indicative results of their performance: Real and imaginary part of the input impedance for specific geometrical and material parameter values

It is worthwhile to mention that by additional modification of the Sierpinski gasket as proposed in[62] or in [69] (see fig. 32), the bands of resonance could be further controlled in order to meet the technical requirements of the applications for which the antenna is designed.

The Sierpinski carpet is another Sierpinski fractal configuration reported in antenna applications. Sierpinski carpet dipole antennas are shown in figures 33 and 34. The study of these configurations guide to the conclusion that no multiband performance can be 
obtained. It is due to the fact that the fractal iterations do not perturb the active current carrying region. So, their performance is similar to that of a simple square patch.

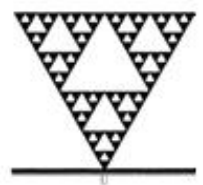

(a)
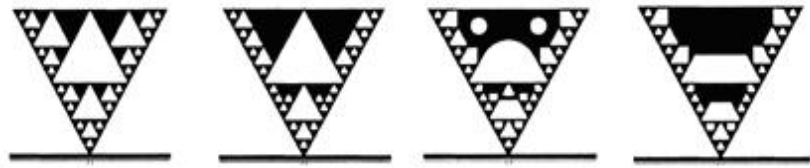

(b)

Fig. 32. Printed multiband antenna monopoles a)The self similar ordinary Sierpinski gasket.

b) Modified Sierpinski gaskets.

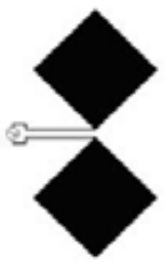

K0

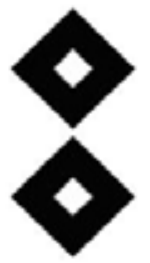

K1

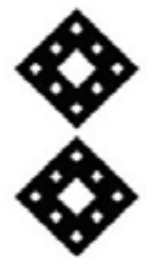

K2

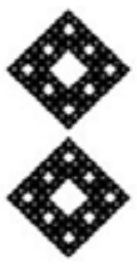

K3

Fig. 33. Sierpinski carpet dipole antennas: the generator and the first three fractal orders

The negative version of the above fractal scheme is shown in Fig. 34. The geometrical method to design this carpet is the following: The generator is that of fig. 34a. In the first iteration the area around the central patch is divided in nine sub-areas of equal size and at the center of each sub-area a rectangular patch with side length three times smaller than the initial central patch is located. The same process is applied in the next iteration. In this antenna only the central element is driven and the energy of the other smaller patches is coupled parasitically from the driven patch.

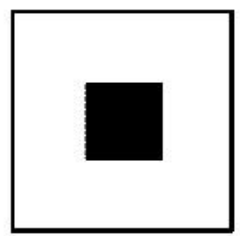

K1

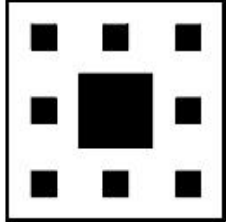

K2

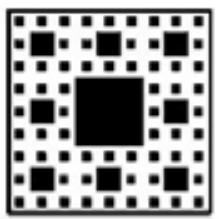

K3

Fig. 34. Sierpinski carpet fractal antennas: the generator and the first two orders.

This fractal microstip configuration exhibits multifrequency performance, Fig. 35, but it was found[63] that the results come from the driven element, not from the parasitic ones.

\subsection{5 c)Hilbert fractals}

The properties of the Hilbert curve make them attractive candidates for use in the design of fractal antennas. These curves apart from being self similar have the additional property of approximately filling a plane and this attribute is exploited in realizing a 'small' resonant antenna. Hilbert fractal antennas with size smaller than $\lambda / 10$ are capable to resonate, with performance comparable to that of a dipole whose resonant length is close to $\lambda / 2$. 


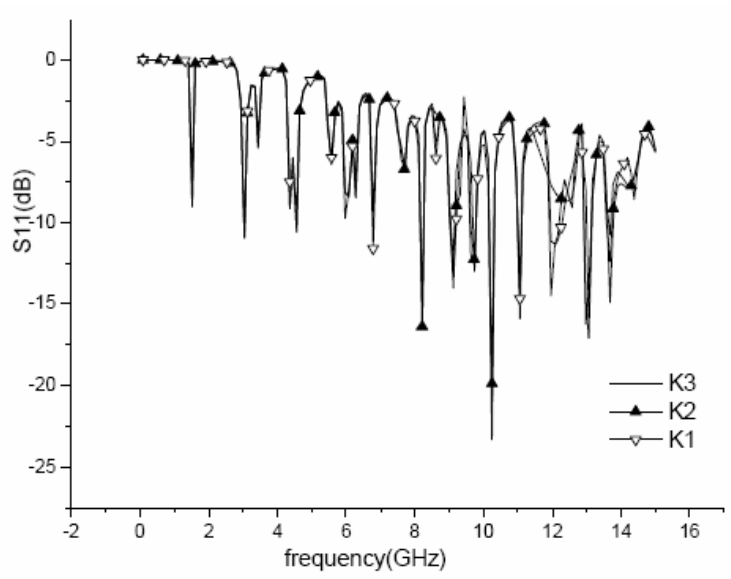

Fig. 35. The reflection coefficients of Sierpinski carpet microstrip antenna(fig. 34) in different iterations

The generator of the Hilbert curve has the form of a rectangular $U$ as shown in fig. 36a. The Hilbert curves for the first several iterations are shown in figures 36b-36d. The construction at a stage is obtained by putting together four copies of the previous iteration connected by additional line segments.

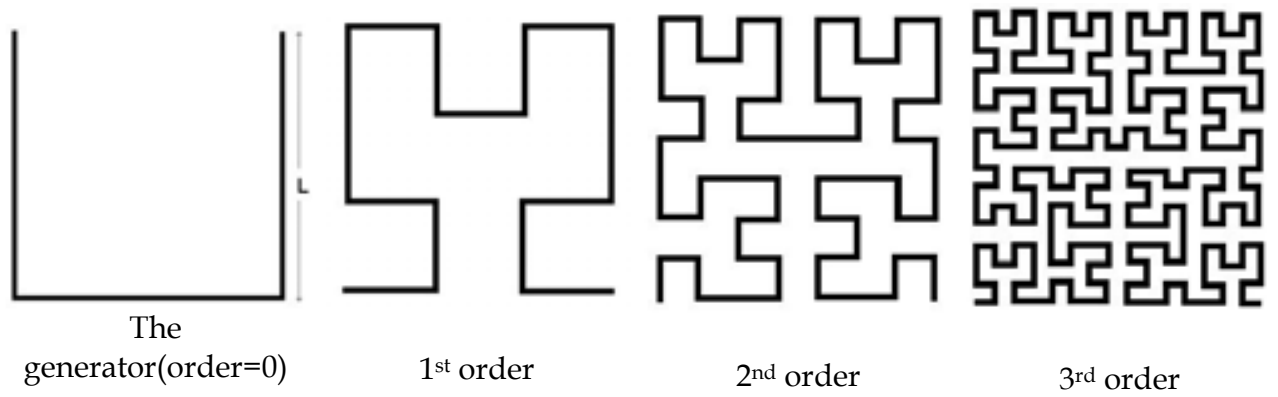

Fig. 36. The Hilbert fractal printed antennas of various stages.

It would be interesting to identify the fractal properties of this geometry. The space-filling nature is evident by comparing the first few iterations shown in figure 36. It may however be mentioned that this geometry is not strictly self similar since additional connection segments are required when an extra iteration order is added to an existing one. But the contribution of this additional length is small compared to the overall length of the geometry, especially when the order of the iteration is large. Hence, this small length can be disregarded which makes the geometry self similar. Moreover the curve is almost filling a plane. In other words the total length, if sum the line segments, tends to be extremely large. This could lead to a significant advantage, since the resonant frequency can be reduced considerably for a given area by increasing the fractal iteration order. Thus, this approach strives to overcome one of the fundamental limitations of antenna engineering with regard to small antennas. 
For an accurate study of the operational features of a Hilbert fractal printed antenna information about its geometric parameters are necessary. It is obvious that as the iteration order increases, the total length of the line segments is increased in almost geometric progression if the outer dimension is kept fixed. Thus, for a Hilbert curve antenna with side dimension $\mathrm{L}$ and order $\mathrm{n}$, the total segment length $\mathrm{S}(\mathrm{n})$ is calculated by the formula

$$
\mathrm{S}(\mathrm{n})=\frac{2^{2 \mathrm{n}}-1}{2^{\mathrm{n}}-1} \mathrm{~L}
$$

and the length of each line segment is given by

$$
\mathrm{d}=\frac{\mathrm{L}}{2^{\mathrm{n}}-1}
$$

A theoretical approach for the calculation of the resonant frequencies of the antenna considers the turns of the Hilbert curve as short circuited parallel-two-wire lines and begins with the calculation of the inductance of these lines[70], [71]. This approach is illustrated in figure 37. The self inductance of a straight line connecting all these turns is then added to the above, inductance multiplied by the number of shorted lines, to get the total inductance. To find the frequencies of resonance, the total inductance is compared with the inductance of a regular half wavelength dipole.

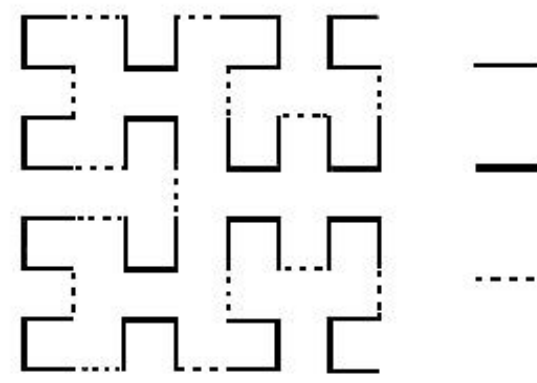

Parallel line section with length d and width $b$

Short circuit terminations

of the oaralle wire sections

Additional line segments

for connections (length d)

Fig. 37. The $2^{\text {nd }}$ order of fractal building. The segments used to connect the geometry of the previous iteration are shown in dashed lines

In detail for a Hilbert curve fractal antenna with outer dimension of $\mathrm{L}$ and order of fractal iteration $\mathrm{n}$, there are $\mathrm{m}=4^{\mathrm{n}-1}$ short circuited parallel wire connections each of length $\mathrm{d}$. Moreover the segments not forming the parallel wire sections amount to a total length of

$$
\mathrm{s}=\mathrm{d}\left(2^{2 \mathrm{n}-1}-1\right)=\frac{\mathrm{L}}{2^{\mathrm{n}}-1}\left(2^{2 \mathrm{n}-1}-1\right)
$$

The characteristic impedance of a parallel wire transmission line consisting of wires with diameter $b$, spacing $d$, are given by

$$
\mathrm{Z}_{\mathrm{o}}=120 \log \left(\frac{2 \mathrm{~d}}{\mathrm{~b}}\right)
$$


The above expression can be used to calculate the input impedance at the end of the each line section, which is purely inductive

$$
Z_{\text {in }}=L_{\text {in }}=\frac{Z_{o}}{\omega} \tan (\beta d)=\frac{Z_{o}}{\omega} \tan \left(\beta \frac{L}{2^{n}-1}\right)
$$

It is noticed that at the $\mathrm{n}^{\text {th }}$ stage of fractal building there are $\mathrm{m}=4^{\mathrm{n}-1}$ such sections.

The self inductance due to a straight line of length $\mathrm{s}$ is

$$
\mathrm{L}_{\mathrm{s}}=\frac{\mu_{\mathrm{o}}}{\pi} \mathrm{s}\left(\log \frac{8 \mathrm{~s}}{\mathrm{~b}}-1\right)
$$

So, the total inductance is

$$
\mathrm{L}_{\mathrm{T}}=\mathrm{L}_{\mathrm{s}}+\mathrm{mL}_{\mathrm{in}}=\frac{\mu_{\mathrm{o}}}{\pi} \mathrm{s}\left(\log \frac{8 \mathrm{~s}}{\mathrm{~b}}-1\right)+\mathrm{m} \frac{\mathrm{Z}_{\mathrm{o}}}{\omega} \tan \left(\beta \frac{\mathrm{L}}{2^{\mathrm{n}}-1}\right)
$$

To find the resonant frequency of the antenna, this total inductance is equated with that of a resonant half-wave dipole antenna with approximate length equal to $\lambda / 2$. Taking into account that regular dipole antennas also resonate when the arm length is a multiple of quarter wavelength we can obtain the resonant frequencies of the multi-band Hilbert curve fractal antenna by the expression

$$
\frac{\mu_{\mathrm{o}}}{\pi} \mathrm{k} \frac{\lambda}{4}\left[\log \left(\frac{8}{\mathrm{~b}} \frac{\mathrm{k} \lambda}{4}\right)-1\right]=\mathrm{m} \frac{120}{\omega} \log \frac{2 \mathrm{~d}}{\mathrm{~b}} \tan (\beta \mathrm{d})+\frac{\mu_{\mathrm{o}}}{\pi} \mathrm{s}\left[\log \left(\frac{8 \mathrm{~s}}{\mathrm{~b}}\right)-1\right]
$$

where $\mathrm{k}$ is an odd integer. It is noticed that this expression does not account for higher order effects and hence may not be accurate at higher resonant modes.

At these antennas the feeding point is located at a place of symmetry or at one end of the curve, thus driving the structure to operate as a monopole antenna. It is noticed that the bandwidth at resonances is generally small, whereas the positions of resonant frequencies can be controlled by perturbing the fractal geometry.

In the basis of the above theory, several applications of this type of fractal antenna have been reported. Antennas that can efficiently operate in the range of UHF, as well as in multiple bands, at 2.43GHz and 5.35GHz, serving Wireless Local Area Networks [71]-[73].

\subsection{5 d)Square Curve fractals}

The design of microstrip antennas by the square curve fractal algorithm can yield radiating structures with multiband operation. The generator of this type of fractal objects is a rectangular ring and as a consequence the curves of the various stages are closed curves. The square curve fractals do not belong to the category of the space filling curves. However the increment of their total length from stage to stage is not significant, thus permitting the antennas to meet the requirement of the small size and at the same time to exhibit an increasing gain in virtue of their increasing length.

The staring point of the construction process is the selection of the size of the generator which is a rectangular ring with side length L(Fig. 38a). At the next step of the recursive process, the four corners of the square ring are used as the center of four smaller squares 
each having side, half that of the main square. Overlapping areas are eliminated. The curve produced by this first iteration is shown in Fig.38b. Following the same algorithm the second stage of the fractal antenna can be derived(Fig. 38c). The building of the higher stages is evident.

The total length of the curve is calculated as follow.

a. generator ring: the perimeter is $4 \cdot \mathrm{L}$

b. $1^{\text {st }}$ stage : each side of the generator is divided in four segments of equal length. Two segments are removed, at each corner and they are replace by smaller squares with side length equal to L/2. So, the length of the curve is equal to the sum of segments, common between generator square and the first recursion, plus the length of the newly added segments. The total length of the two segments removed at each corner, is $\mathrm{L} / 2$, so the total removed is $(\mathrm{L} / 2) \cdot 4$. Looking only at the added segments the length increase of the curve is $\mathrm{L}_{\mathrm{st} 1}^{\mathrm{inc}}=(\mathrm{L} / 2) \cdot 8$.

c. $2^{\text {nd }}$ stage: On the second iteration, the corners of the four small squares added at the first iteration are replaced by four even smaller squares with side length $L / 4$. Here the length of the segments removed at each square corner is equal to $\mathrm{L} / 4$ and the length of the smaller squares added is equal to $(\mathrm{L} / 4) \cdot 3$. Taking into account only the added segments the length increase is $\mathrm{L}_{\mathrm{st1}}^{\mathrm{inc}}=(\mathrm{L} / 4) \cdot 24$.

The general formula for the length increase is $\mathrm{L}^{\mathrm{inc}}=\left(\mathrm{L} / 2^{\mathrm{n}}\right) \cdot 8 \cdot 3^{(\mathrm{n}-1)}$ where $\mathrm{n}$ is the iteration number .

The total curve length is $L_{1} \cdot\left(\left(r^{n} \cdot 8\right)-4\right)$, where $r$ is the ratio of the length increase between two sequential iterations.

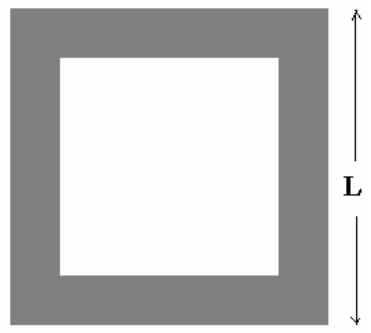

(a)

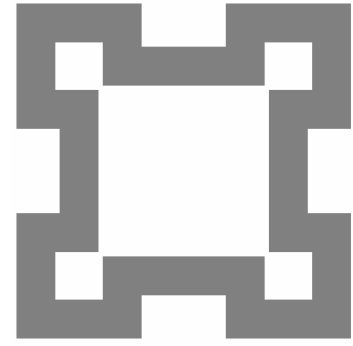

(b)

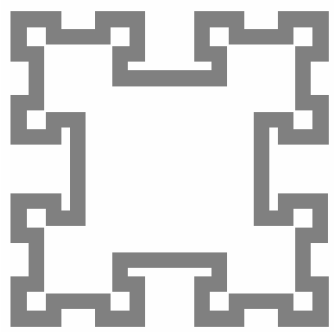

(c)

Fig. 38. The Square Curve fractal a) the generator and (b)-(c) the lower two stages

$\operatorname{In}[75]$ a microstrip fractal structure designed with the aforementioned algorithm is proposed. It was printed on a dielectric substrate with $\varepsilon_{\mathrm{r}}=1.046$ ( Rohacell 51HF, Northern Fiber Glass Service, Inc.) and height $h=1 \mathrm{~mm}$. A value for $r$ equal to 1.5 and the second stage of development gave an object with outer dimensions $8.4 \mathrm{~cm} \times 8.4 \mathrm{~cm}$. A fundamental parameter of the structure is the width of the printed strip which forms the curve. Attention must be paid to the proper selection of the values of the strip's width because there is a trade off between this value and the input impedance of the antenna. A narrow strip guides to high input impedance and inserts difficulties to the matching of the antenna. On 
the other side a wider strip would yield input impedance suitable for direct matching but could produce difficulties related with the space filling during the process of the fractal expanding. More over, the keys to drive this antenna in multi-band operation are the proper number and positions of the feeding points. The incorporation of a pin can also enhance the performance of the antenna.

In figure 39, results received using three different feedings are depicted. Figure 39a shows the variation of the scattering coefficient at the feeding input using one probe, positioned at a point on an axis of symmetry. This choice is common at many fractal antennas. It is observed that only two frequency bands give scattering coefficient lower than $-10 \mathrm{~dB}$. It is due the high input impedance of the antenna, as shown in figure 39b. A better performance with seven frequency bands is obtained with two probes(Fig. 39b); and an even satisfactory operation is achieved when a shorting pin is installed between the probes. The pin

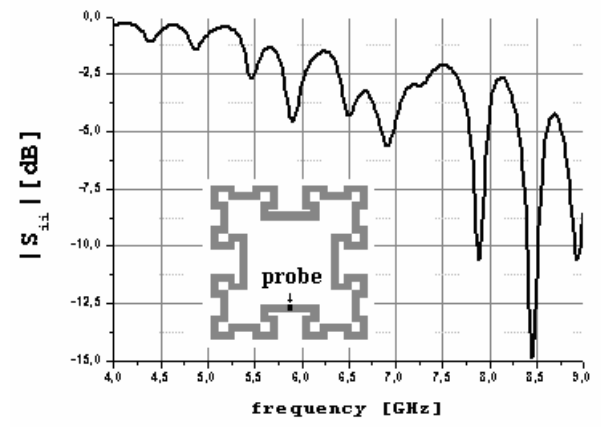

(a)
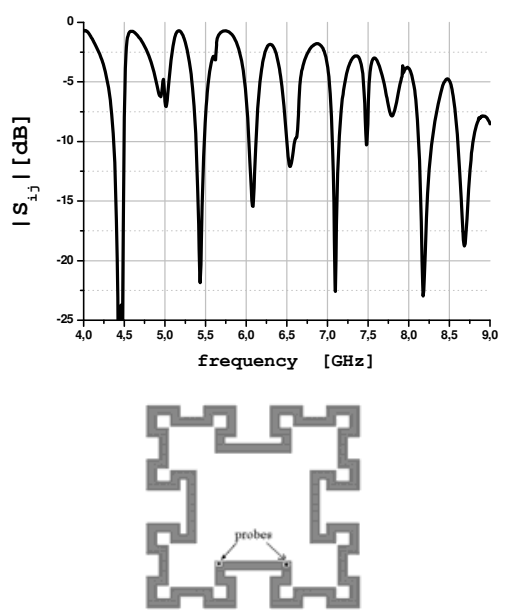

(c)

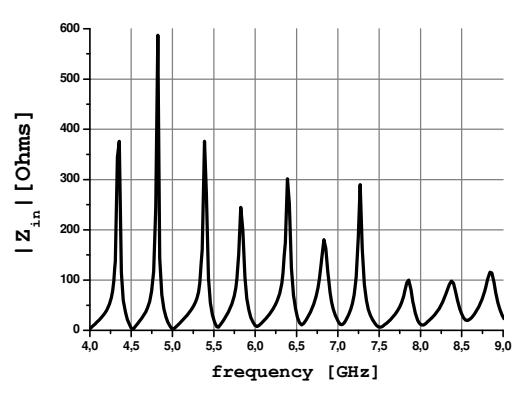

(b)
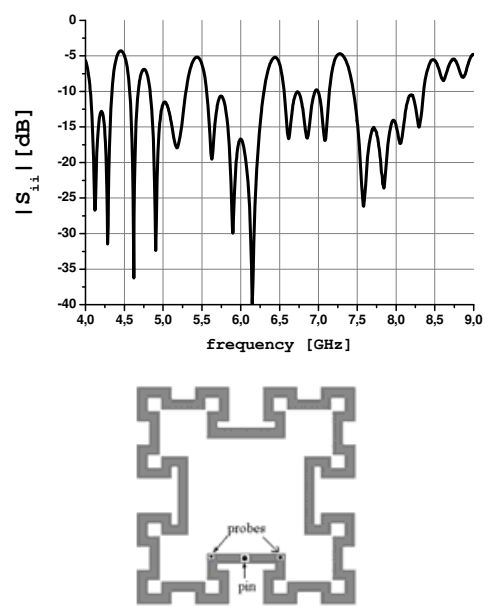

(d)

Fig. 39. a) Scattering coefficient at the input of the second stage fractal antenna fed with one probe and b) the respective input impedance. c) Scattering coefficient when fed with two probes and d)fed with two probes and loaded with one pin. 
acts as a short circuit between the trace and the ground plane, reflecting the wave produced by the probe. So, two complex impedances combined in parallel appear at the point of the feed. The one is due to the line between the probe and the pin and the other to the remaining trace of the structure. These two parallel impedances involve a lower total impedance which would be suitable for direct match to an $75 \mathrm{Ohm}$ probe. Moreover, this matching is attainable in wide ranges around the frequencies of resonance. The results of fig 39c, for the scattering coefficient, show a multiband - and at the same time wideband operation.

\section{Electromagnetic Bandgap Structures (EBG) in antenna applications}

\subsection{The EBG structure and properties}

Electromagnetic Band Gap (EBG) structures constitute a specific class of recently discovered microwave objects that, due to their special electromagnetic behavior, reveal promising solutions to several microwave problems, especially in the area of communications[76],[77]. The EBG structures are generally defined as 'artificial periodic or non periodic objects that prevent the propagation of electromagnetic waves in a specified band of frequency for all incident angles and all polarization states'. They can be categorized into three groups according to their geometric configuration: Three dimensional volumetric structures, twodimensional planar surfaces and one dimensional transmission lines. Among these three categories the planar EBG objects are the most commonly used in antenna systems. They consist of a two dimensional lattice of metal plates conductively connected to a ground plane by metal-plated vias, as shown in figure 40 , and are easily fabricated using printed circuit board technology.

The effective application of EBG surfaces to the antenna design is based on the exploitation of their distinctive electromagnetic properties with respect to the type of the incident electromagnetic waves:

i. When a plane wave impinges on an EBG surface it is reflected with a phase that varies with frequency as shown in figure 41 . At a certain frequency the reflection phase is zero degrees. The value of this frequency depends on the structural parameters of the EBG object. This performance resembles a perfect magnetic conductor that does not exist in the nature.

ii. When the incident wave is a surface wave the EBG structures show a frequency band gap through which the surface wave cannot propagate for any incident angles and polarization states.

Both the above attributes contribute to the enhancement of the performance of printed or not printed antenna elements or arrays of elements. For example in the case of a microstrip antenna integrated with EBG structures, the suppression of the surface waves could reduce the mutual coupling between the antenna elements, if the antenna is an array, and also prevent the wave to reach the boundaries of the configuration and be diffracted. This prevention involves lower radiation towards the back space of the antenna, an attribute that would ensure low interference with adjacent microwave elements or low radiation towards the user of the equipment that hosts the antenna. Moreover, due to the property of an EBG cell to work as a resonator, enhancement of the antenna gain could be obtained and also an easier and effective matching of the system to the feeding probe. Furthermore an EBG object could drive the microstrip antenna to a dual frequency operation modifying the higher order radiation patterns, thus making them similar to those of the basic mode. On the 
other hand an EBG surface is a unique object to obtain low profile antennas if the radiating element is not a microstrip antenna but a wire dipole, thus providing simple and effective radiation systems.

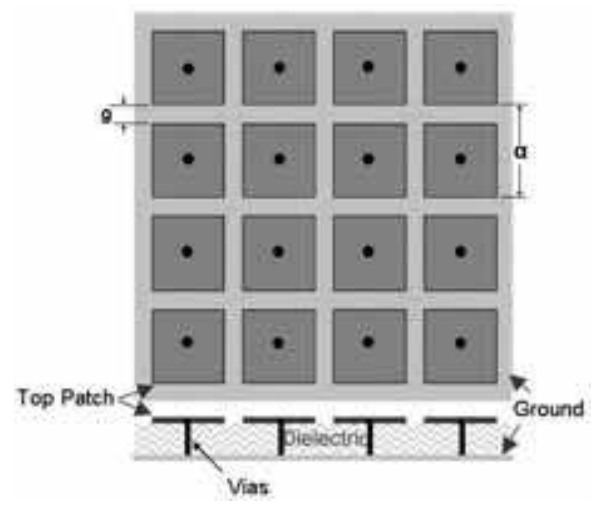

Fig. 40. Geometry of the mushroom-like EBG structure

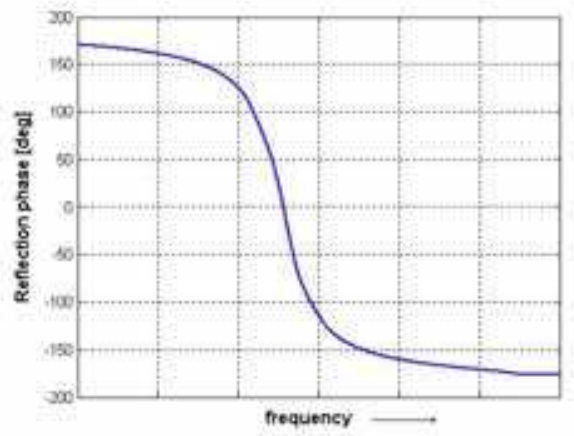

Fig. 41. The phase of a plane wave reflected by the EBG surface

A theoretical analysis of an EBG structure, via various models, gets an insight into the way by which they perform and can yield mathematical expressions for their operational parameters. The analysis would explain the mechanisms by which the EBG objects enhance the performance of the antennas and would give to the antenna designer the ability to properly exploit all the EBG properties, potentially useful in a specific antenna application.

\subsection{Theoretical analysis}

\subsubsection{Low and high impedance surfaces}

Flat metal sheets have low surface impedance and are used in many antennas as a reflector or a ground plane. The boundary conditions on these surfaces impose that the tangential component of the electric field intensity has to be equal to zero and this requirement involves that the metal sheet reflects an impinging wave, shifting the phase of its intensity by an amount of п. Moreover the metal sheet redirects one-half of the radiation into the opposite direction improving the antenna gain by $3 \mathrm{~dB}$ and partially shielding objects on the other side. However, if the antenna is too close to the conductive surface, the out of phase 
image currents 'cancel' the currents in the antenna, resulting in poor radiation efficiency. This problem is often addressed by positioning the radiating element at a quarterwavelength distance from the ground plane but this arrangement requires minimum thickness of $\lambda / 4$.

By incorporating a special texture on a conducting surface it is possible to alter its radiofrequency electromagnetic properties. A proper modification (see for example fig. 40)would yield a specific surface with high surface impedance. On these textured surfaces the tangential component of the magnetic field intensity tends to zero and this condition means that the surface reflects an incident wave with an almost zero phase shift. This minimization of the magnetic field is due to the minimization of the surface currents, that inevitably comes from the cutting up of the metallic surface, into small patches. It is noticed that although the magnetic field intensity is very small, the electric field may have a large value due to the high voltage induced between the edges of the adjacent patches of the modified sheet. The edges and the narrow gaps between them realize capacitors. The ratio of the high electric field intensity, which is high, over the low magnetic field intensity defines the impedance of the surface which, in this case, is obviously very high. The almost zero tangential magnetic field permit us to term the surface as an artificial magnetic conductor. This unusual boundary condition involves that the image currents are in phase rather than out of phase, allowing radiating elements to lie in very close proximity to the surface while still radiating efficiently.

As a consequence of the performance described above, this type of high impedance surface can function as a new type of ground plane for low profile antennas. For example a dipole positioned in parallel to a high impedance ground plane is not shorted out as it would be on an ordinary metal sheet. In addition to their unusual reflection-phase properties, these textured structures have a surface wave bandgap, within which they do not support bound surface waves of either transverse magnetic (TM) or transverse electric(TE) polarization. They may be considered as a kind of electromagnetic bandgap structures or photonic crystals for surface waves. It is noticed that although bound surface waves are not supported, leaky TE waves can propagate within the bandgap, and they are useful for certain applications.

The theoretical basis to explain the electromagnetic behavior of an EBG structure is the general theory of surface waves and the useful tool for the description of its performance is the surface impedance of the EBG object along with equivalent electric circuit of this impedance.

\subsubsection{Surface waves}

Surface waves can occur on the interface between two dissimilar materials, for example metal and free space[76]-[78]. They are bound to the interface and decay exponentially into the surrounding materials although at radio frequencies the fields associated with these waves can extend thousands of wavelengths into the surrounding space. To describe theoretically and by a simple manner their physical entity, let us suppose an interface parallel to yz plane as shown in figure 42 .

Assume a wave that is bound to the surface in the $+x$ direction with decay constant $\alpha$, and in $-x$ direction with a decay constant $\gamma$. The wave propagates in the $z$-direction with propagation constant $\mathrm{k}$. For a TM polarized surface wave $\mathrm{E}_{\mathrm{y}}=0$. The electric field in the upper half-space has the following form 


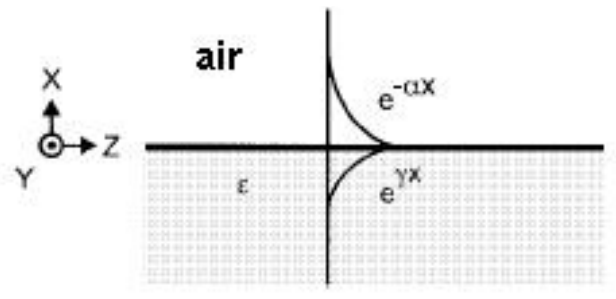

Fig. 42. A surface wave is bounded around the interface of two media and decays exponentially into the surrounding space

$$
\overrightarrow{\mathrm{E}}_{1}=\left(\hat{\mathrm{x}} \mathrm{E}_{1 \mathrm{x}}+\hat{\mathrm{z}} \mathrm{E}_{1 \mathrm{z}}\right) \mathrm{e}^{\mathrm{j \omega t}-\mathrm{jkz}-\alpha \mathrm{x}}
$$

In the lower half-space the electric field is

$$
\overrightarrow{\mathrm{E}}_{2}=\left(\hat{\mathrm{x}} \mathrm{E}_{2 \mathrm{x}}+\hat{z} \mathrm{E}_{2 z}\right) \mathrm{e}^{\mathrm{j} \omega \mathrm{t}-\mathrm{jkz}-\gamma \mathrm{x}}
$$

The solution of Maxwell equations yields the following results for the propagation constants

$$
\begin{aligned}
& \mathrm{k}=\frac{\omega}{\mathrm{c}} \sqrt{\frac{\varepsilon}{1+\varepsilon}} \\
& \alpha=\frac{\omega}{\mathrm{c}} \sqrt{\frac{-1}{1+\varepsilon}} \\
& \gamma=\frac{\omega}{\mathrm{c}} \sqrt{\frac{-\varepsilon^{2}}{1+\varepsilon}}
\end{aligned}
$$

If $\varepsilon$ is real and positive, then $\alpha$ and $\gamma$ are imaginary and the waves do not decay with distance from the surface and propagate through the dielectric interface. Thus TM surface waves do not exist on nonconductive dielectric materials. On the other hand if $\varepsilon$ is less than 1 , or if it is imaginary or complex, the solution describes a wave that is bound to the surface. These TM surface waves can occur on metals or other materials with non-positive dielectric constants.

The complex permittivity of a metal can be expressed in the following form

$$
\dot{\varepsilon}=\varepsilon_{\mathrm{o}} \varepsilon_{\mathrm{r}}\left(1-\mathrm{j} \frac{\sigma}{\omega \varepsilon_{\mathrm{o}} \varepsilon_{\mathrm{r}}}\right)
$$

where $\sigma$ is the conductivity of the metal, $\varepsilon_{0}$ is the permittivity of the free space and $\varepsilon_{\mathrm{r}}$ is the dielectric constant.

For relatively low frequencies, including the microwave spectrum, the conductivity is primarily real and much greater than unity, thus, the permittivity is a large imaginary number which, supposing that $\varepsilon_{\mathrm{r}}=1$, is calculated by $\dot{\varepsilon} \approx \mathrm{j} \frac{\sigma}{\omega \varepsilon_{0}}$ and in accordance to (36a) 
the dispersion relation for surface waves at radio frequencies is approximately $\mathrm{k} \approx \frac{\omega}{\mathrm{c}}$.

Thus, surface waves propagate at nearly the speed of light in the vacuum and they travel for many wavelengths along the metal surface with little attenuation.

The decay constant of the fields into the surrounding space is derived by inserting (37) into (36b). It is easy to ascertain, by an arithmetic application at microwave frequencies, that the surface waves extend a great distance into the surrounding space.

Constant $\gamma$, the inverse of which is related to the wave penetration depth into the metal, is approximately

$$
\gamma \approx(1+\mathrm{j}) \sqrt{\frac{\omega \mu_{0} \sigma}{2}}=\frac{(1+\mathrm{j})}{\delta}
$$

where $\delta$ is the skin depth. The surface currents penetrate only a very small distance into the metal. They can be expressed in terms of the skin depth, the conductivity and the electric field intensity and can be used for the determination of the magnetic field intensity. Then, the surface impedance is derived by the electric and magnetic field intensities as follows

$$
\mathrm{Z}_{\mathrm{s}}=\frac{\mathrm{E}_{\mathrm{z}}}{\mathrm{H}_{\mathrm{y}}}=\frac{1+\mathrm{j}}{\sigma \delta}
$$

The above results show that the surface impedance has equal positive real and positive imaginary parts, so the small surface resistance of the metal surface is accompanied by an equal amount of surface inductance.

By texturing the metal surface we can alter its surface impedance and thereby change its surface-wave properties. Thus the surface impedance would appear inductive or capacitive imaginary part, depending on the frequency. The derivation of the impedance is made considering that a wave decays exponentially away from the boundary with a decay constant $a$, whereas the boundary is taken into account by its surface impedance. In this case it has been proved that TM waves occur on an inductive surface, in which the surface impedance is given by the following expression

$$
\mathrm{Z}_{\mathrm{S}}^{\mathrm{TM}}=\frac{\mathrm{j} \alpha}{\omega \varepsilon}
$$

Correspondingly, TE waves can be excited on a capacitive surface. In this case the surface impedance is

$$
\mathrm{Z}_{\mathrm{S}}^{\mathrm{TE}}=\frac{-\mathrm{j} \omega \mu}{\alpha}
$$

The wave vector $k$, in terms of the frequency and the decay constant $a$, helps to get an insight into the behavior of the surface

$$
\mathrm{k}^{2}=\mu_{0} \varepsilon_{0} \omega^{2}+\alpha^{2}
$$


Combining equation (42) with the equations (40) and (41) we can derive the dispersion relation for TM waves

$$
\mathrm{k}^{\mathrm{TM}}=\frac{\omega}{\mathrm{c}} \sqrt{1-\frac{\left(\mathrm{Z}_{\mathrm{S}}^{\mathrm{TM}}\right)^{2}}{\eta^{2}}}
$$

And for TE waves

$$
\mathrm{k}^{\mathrm{TE}}=\frac{\omega}{\mathrm{c}} \sqrt{1-\frac{\eta^{2}}{\left(\mathrm{Z}_{\mathrm{S}}^{\mathrm{TE}}\right)^{2}}}
$$

where $\eta=\sqrt{\frac{\mu_{0}}{\varepsilon_{0}}}$ is the impedance of the free space.

The complete dispersion diagrams of $k$ versus frequency (Fig. 43), show that for a specific textured surface there is a certain frequency, termed resonance frequency, below of which TM surface waves are supported. At frequencies much lower than the resonance frequency the dispersion curve is very near to the light line, revealing the propagation of the wave, thus the field extends many wavelengths along the surface, as does on a metal sheet and the surface is considered of low impedance. Near the resonance the surface waves are tightly bound to the surface and, as the slope of the curve is very small, the group velocity is low. All these mean the surface impedance of the structure becomes high. Above the resonance frequency, the surface appears a capacitive behavior and TE waves are supported. Near the resonance frequency the TE waves are weakly bound to the surface extending far into the surrounding space. As the frequency increases the curve bends away from the light line and it means that the waves are more tightly bound to the surface.

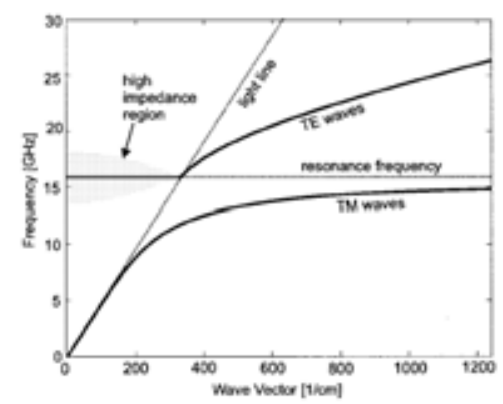

Fig. 43. The predicted surface wave dispersion diagram

\subsubsection{Equivalent circuit model}

They are exactly the above features of the propagating waves that permit us to approach the electromagnetic behavior of the EBG surface using an equivalent parallel resonant circuit which can be tuned to exhibit high impedance over a pre-specified frequency band. From the physical side of view this equivalence can be explained as follows: as the EBG interacts 
with electromagnetic waves, currents are induced in the top metal plates. A voltage applied parallel to the top surface causes charges to concentrate around and on the ends of the plates which can be considered as a capacitance. As the charges move back and forth, they flow around a long path through the vias and the bottom plate(Fig. 44). Associated with these currents is a magnetic field and, thus, an inductance.
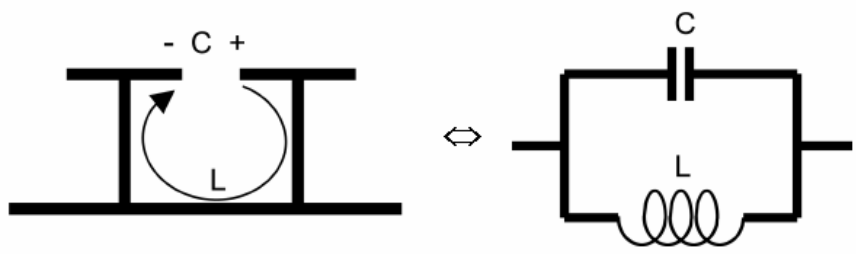

Fig. 44.

By this circuit model, the surface impedance, assigned to the sheet, corresponds to the impedance of a parallel resonant circuit, consisting of the sheet capacitance and the sheet inductance

$$
Z=\frac{j \omega L}{1-\omega^{2} L C}
$$

The surface is inductive at low frequencies, capacitive at high frequencies and the impedance is very high near the resonance frequency $\omega_{0}$,

$$
\omega_{0}=\frac{1}{\sqrt{\mathrm{LC}}}
$$

This high impedance is associated with a forbidden frequency band. In the 2D geometry of the lattice of the EBG patches the capacitors are formed by the fringing electric fields between adjacent metal patches and the inductance is fixed by the thickness of the structure. An approximate expression for the capacity [79], in the case of the geometry shown in fig. 40 , is equation (47), [79]

$$
\mathrm{C}_{\text {fringe }} \approx \frac{\mathrm{w}\left(\varepsilon_{1}+\varepsilon_{2}\right)}{\pi} \cosh ^{-1}\left(\frac{\alpha}{\mathrm{g}}\right)
$$

In the above expression $\mathrm{g}$ is the gap between the plates, $\mathrm{w}$ is the width of the plates, $\alpha$ is the lattice constant namely $\alpha=\mathrm{g}+\mathrm{W}$, and $\varepsilon_{1}$ and $\varepsilon_{2}$ are, respectively, the dielectric constants of the substrate of the EBG and the material surrounding the surface which may be free space. Equation (47), although approximate, is adequate for first order designs. The inductance of a high -impedance surface is determined entirely by its thickness. This can be understood by considering a solenoid of current that includes two rows of plates and their associated vias. Current flows up one row of vias across the capacitors and down the next set of vias to return through the ground plane. The length and width of the solenoid are canceled to obtain the sheet inductance, $\mathrm{L}$

$$
\mathrm{L}=\mu \mathrm{h}
$$


For the effective surface impedance approximation to be valid the lattice constant should be small compared to the wavelength.

Another, also approximate, equation for $C$ is proposed in [80]. It was produced considering normally-incident plane waves and the vias conductors, connecting patch to the ground, not excited. In this case also square patches, small compared with the wavelength, and gaps between the patches, very narrow compared with the length side of the patches were considered. In the used model the higher order Floquet modes generated by the periodical mesh were neglected. Assuming only the fundamental mode plane waves between the lattice of patches and the ground, the equivalent surface impedance was considered as the parallel connection of two impedances: the impedance of the lattice and the input impedance of a TEM line section of length $h$. The expression of the surface impedance, normalized to the free space impedance was found

$$
\mathrm{Z}_{\mathrm{sn}}=\frac{\mathrm{j} \frac{1}{\sqrt{\varepsilon_{\mathrm{r}}}} \tan \left(\omega \mathrm{h} \sqrt{\varepsilon_{\mathrm{r}} \varepsilon_{0} \mu_{0}}\right)}{1-\frac{\left(\varepsilon_{\mathrm{r}}+1\right)\left(\omega \alpha \sqrt{\varepsilon_{0} \mu_{0}}\right)}{\pi \sqrt{\varepsilon_{\mathrm{r}}}} \log \left(\frac{2 \alpha}{\pi \mathrm{g}}\right) \tan \left(\omega \mathrm{h} \sqrt{\varepsilon_{\mathrm{r}} \varepsilon_{0} \mu_{0}}\right)}
$$

Where $\alpha$ and $g$ were defined previously. The equation for the capacitance $C$ is

$$
\mathrm{C}=\frac{\mathrm{D} \varepsilon_{0}\left(\varepsilon_{\mathrm{r}}+1\right)}{\pi} \log \left(\frac{2 \alpha}{\pi \mathrm{g}}\right)
$$

\subsubsection{Reflection phase and Bandwidth}

Besides their unusual surface wave features, the high impedance surfaces also have unusual reflection phase properties. Using the effective surface impedance model, described previously, we can determine the reflection phase for the resonant textured surface. For a normally incident wave, the reflection phase of the surface is given as

$$
\Phi=\operatorname{Im}\left[\ln \left(\frac{Z_{s}-\eta}{Z_{S}-\eta}\right)\right]
$$

where $Z_{S}$ is the surface impedance and $\eta=120 \pi$ is the free space impedance. At very low frequencies the reflection phase is $\Pi$ (see fig. 41) and the structure behaves like a smooth metal surface. At higher frequencies the reflection phase slopes downward and crosses through zero at the resonance frequency. This situation corresponds to the artificial magnetic conductor behavior. Above the resonance frequency the phase converges to $-\Pi$. The phase varies between $\Pi / 2$ and $-\Pi / 2$ when the magnitude of the surface impedance exceeds the impedance of the free space. It is worth noting that for a wide range of geometries the bounds of the surface' $s$ wave band gap, occur at the same frequencies where the reflection phase crosses through $\Pi / 2$ and $-\Pi / 2$.

To determine the bandwidth of an antenna positioned in parallel to an EBG surface, its radiation is modeled as a resistor, valued the impedance of free space, in parallel to the LC equivalent circuit of the EBG lattice. The amount of power dissipated in the resistor is a 
measure of the radiation efficiency of the antenna. The maximum radiation efficiency occurs at the LC resonance frequency of the EBG surface, where the surface reactance is infinite. At very low or at very high frequencies, the radiated power is reduced. It can be shown that the frequencies where the radiation become equal to half of its maximum value, occur when the magnitude of the surface impedance is equal to the impedance of free space. It is easily proved that the frequencies at the bounds of the operating band are approximately given by the expressions

$$
\omega \approx\left(1 \pm \frac{1}{\mathrm{Z}_{0}} \sqrt{\frac{\mathrm{L}}{\mathrm{C}}}\right)
$$

The two frequencies designated by the plus and minus signs delimit the frequency range over which an antenna would radiate efficiently on such a surface. The relative bandwidth, defined as the ratio of $\Delta \omega$ over the frequency of resonance, is approximately equal to

$$
\mathrm{BW}=\frac{\Delta \omega}{\omega_{0}} \approx \sqrt{\frac{\mathrm{L} \varepsilon_{0}}{\mathrm{C} \mu_{0}}}
$$

It is the bandwidth, over which the phase of the reflection coefficient falls between $\Pi / 2$ and $\Pi / 2$ and image currents are more in phase than out of phase. As noted in the previous section, this range often coincides with the surface wave band-gap.

The geometry of the EBG and the parameters of the materials can be adjusted to provide the desired resonance frequency and the bandwidth and it is usually done via simulations. However it is useful to have an initial solution to more rapidly converge on the correct design. So, to design an EBG surface for pre-specified frequency of resonance $\omega_{0}$ and bandwidth BW, in accordance to the aforementioned analysis the thickness of the substrate is determined by the equation

$$
\mathrm{h}=\frac{\mathrm{cBW}}{\omega_{0}}
$$

and the equation for the required sheet capacitance is

$$
C=\frac{1}{\omega_{0} \eta B W}
$$

It is worth noting the there is a relation between the bandwidth and the variation of the phase reflection. The rate by which the reflection phase varies, conditions the frequency bandwidth of the mushroom type EBG and depends on the length of the side of the patch, the width of the gap between the patches, the dielectric constant and the thickness of the substrate[81].

Diagrams illustrating this performance are depicted in figure 45. In fig. $45 \mathrm{a}$ is shown that the increment of the side length reduces the frequency of resonance, as expected, but also reduces the bandwidth. From fig. 45b it is concluded that when the gap width is increased, both the frequency band position and its bandwidth increase. Fig. 45c shows that when the substrate permittivity $\varepsilon_{\mathrm{r}}$ is increased the frequency band position and the bandwidth 
decrease. The results of fig. $45 \mathrm{~d}$ prove that when the substrate thickness is increased the frequency band position decreases while its bandwidth increases.

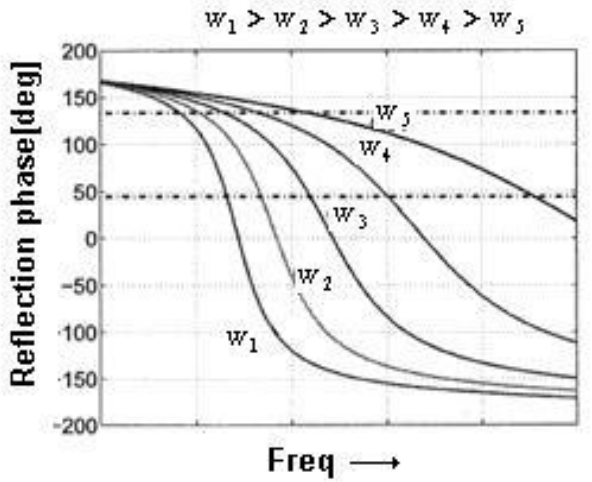

(a)

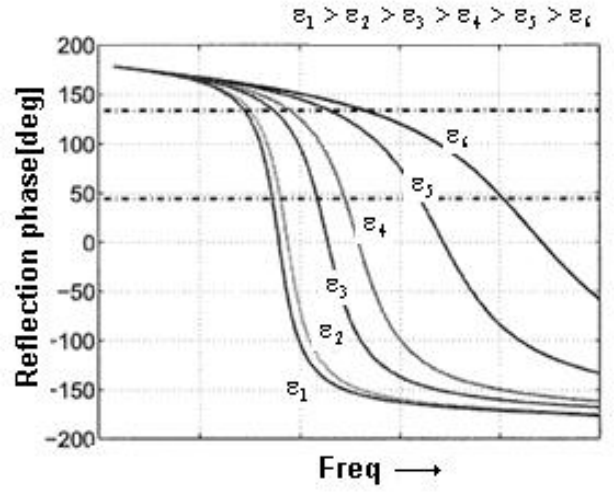

(c)

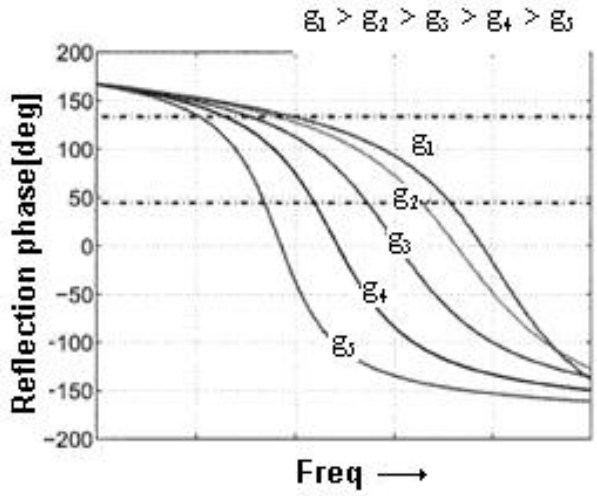

(b)

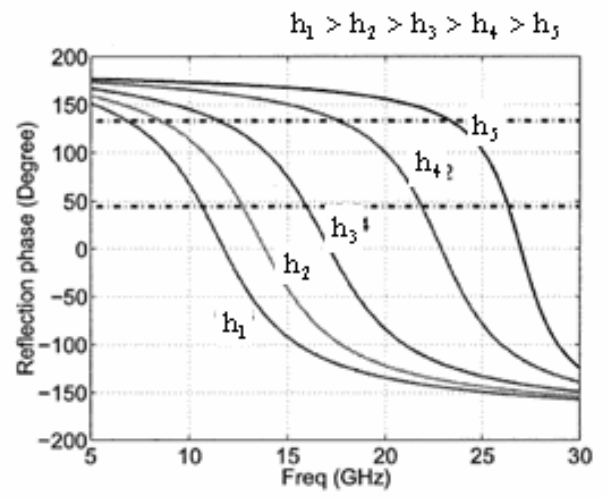

(d)

Fig. 45. EBG parameters analysis. Reflection phase variation versus frequency. Effects of a) patch width b)gap between the patches of the EBG lattice c) substrate permittivity d)substrate thickness.

\subsection{EBG structures in antenna applications}

Although the mushroom type cell is the most commonly used, various EBG types of cells (fig. 46) have been proposed for the enhancement of the antennas they host.

Whatever the type of the used EBG is, the determination of its proper configuration for the improvement of the antenna performance is not an easy task. The band-gap of an EBG, designed in advance, cannot guarantee the effective operation of the antenna that will be incorporated in the following, because complicated interactions occur between the antenna and the EBG lattice. Due to these interactions the improvement of the operation of the system antenna-EBG, as well as the satisfactory feed matching of the antenna, are problems that must be simultaneously solved. An efficient strategy for this purpose is the parametric study of the entire configuration, which is supported by simulations via a high frequency 
electromagnetic software, before the manufacturing the antenna system. Alternative ways to solve the problem is the employment of deterministic or stochastic algorithms, as will be discussed in the next paragraph.

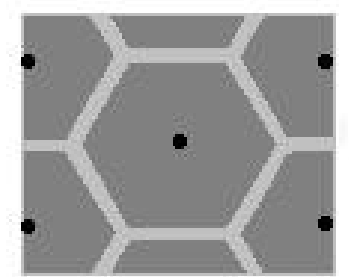

Honeycomb

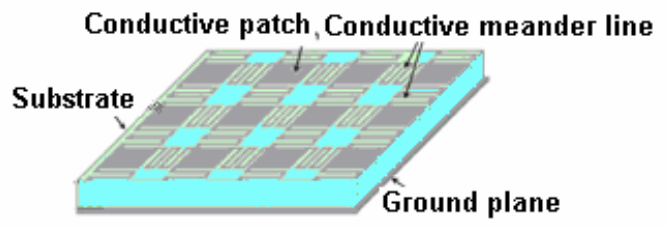

Rectangular patches conductively connected with meander strip lines

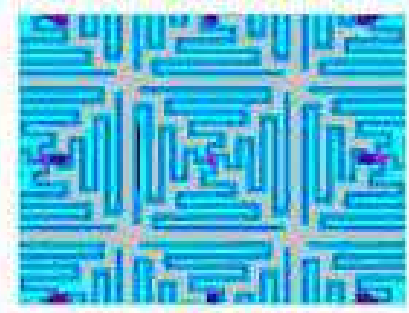

Winding Cross

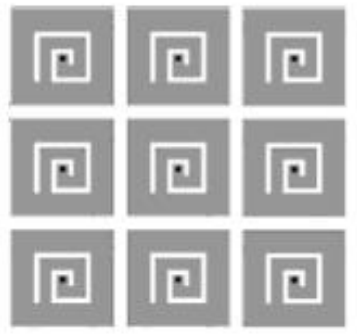

Spiral slotted rectangular patches

Fig. 46. Various printed schemes suitable for EBG implementation

Many articles have reported the contribution of the EBG surfaces to the enhancement of the operation of antennas incorporated in their structure. Some typical applications are referred in the following.

In [82] a structure consisted of a 'diamond dipole' of two square patches over a mushroom type EBG was studied(Fig. 42a). The suitable parameter values of the scheme yield the results shown in fig. $47 \mathrm{~b}$. The presence of the EBG drives the dipole to operate effectively within a bandwidth much wider than that of the ordinary metallic ground sheet.

The configurations shown in figures 48 and 49 concern in antenna array arrangements, in which reduction of mutual coupling between their elements was obtained, via EBG schemes.

Fig. $48 \mathrm{~b}$ depicts the scattering parameter $S_{12}$ between the printed elements in the arrangement shown in fig. 48a. The significant reduction of $S_{12}$ in the band of EBG resonance, proves the effectiveness of the EBG lattice, embedded between them, in reducing their mutual coupling[83].

In [84] an antenna array of microstrip elements with high directivity was designed. A large directivity can be obtained by an array with large size and it means that the distance between the elements has to be large. However the large distance would involve undesirably high side lobes. So, to obtain high directivity the designers chose to keep the inter-element distance small and to increase the size of the patch. Small inter-element distance causes strong mutual coupling between the elements of the array. This problem was confronted inserting an EBG configuration between adjacent elements as shown in 


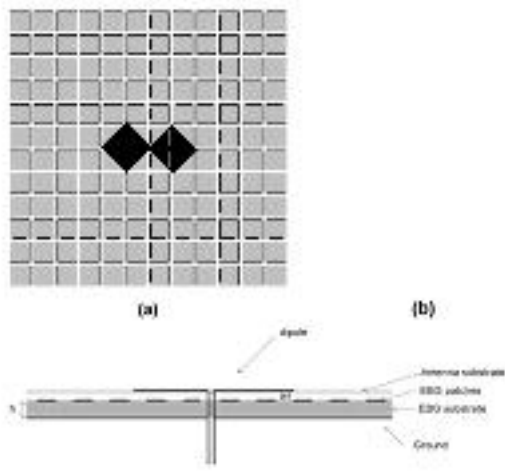

(a)

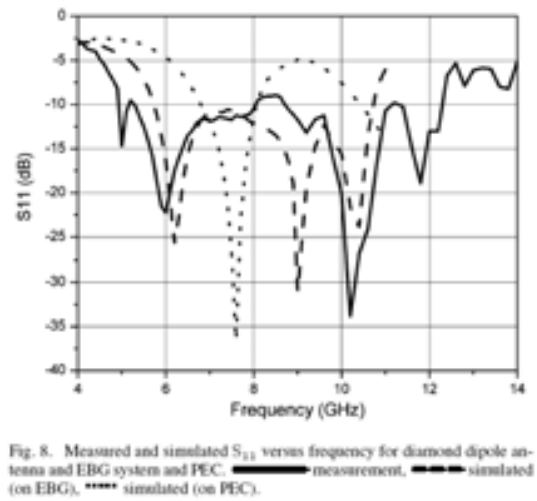

(b)

Fig. 47. a)Top and side view of a diamond planar dipole antenna over a mushroom type EBG $b$ ) the broadband performance of the structure: scattering coefficient of the signal at the feeding probe.

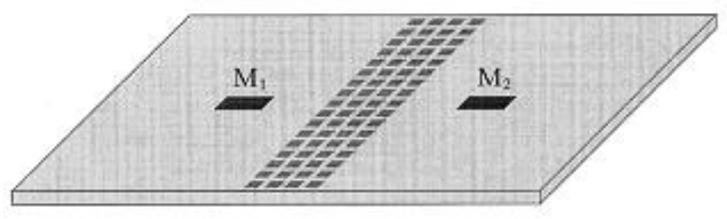

(a)

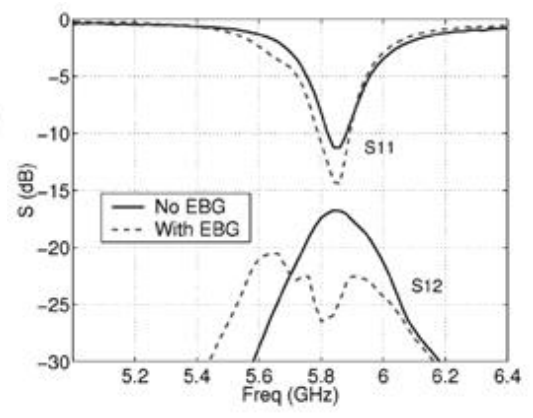

(b)

Fig. 48. a)Microstrip antenna separated by the mushroom-like EBG b) Mutual coupling between the patches with and without EBG

Fig.49a. The increasing of the patch's size was achieved using a dual layer dielectric substrate. A large permittivity was selected for the lower layer while the upper layer was the air. The additional advantage of this configuration is that a substrate with large thickness and low effective permittivity was created and as a result the bandwidth was enhanced. Moreover the patches of the EBG printed on the top of the lower layer with high permittivity had size enough small, to be placed between the antenna radiating patches. In the proposed array, by proper selection of the structural parameter values, a reduction of mutual coupling about $10 \mathrm{~dB}$ or $15 \mathrm{~dB}$, depending on the frequency bandwidth, was obtained.

Another representative example of mutual coupling reduction using EBG in a microstrip antenna array, was recently proposed in [85]. In this configuration (fig. 49b) the thickness of the substrate was large and this fact permitted to embed inside it an elongated mushroom EBG lattice(fig. 49c). By this novel configuration, isolation exceeding $12 \mathrm{~dB}$, was obtained between the antenna array's elements. 


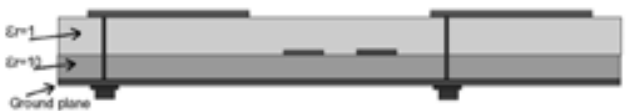

(a)

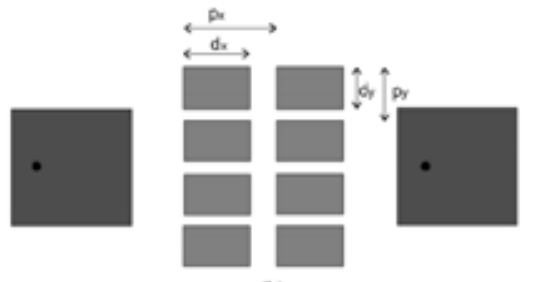

(b)

(a)

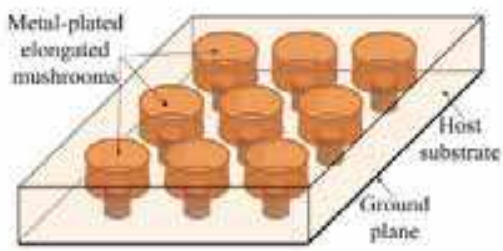

(b)

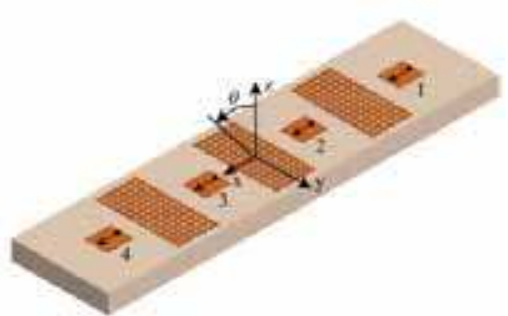

(c)

Fig. 49. Printed antennas integrated with EBG Structurs for mutual coupling reduction: a) Array of two patches structured in a dual-layer substrate. The EBG embedded between the patches reduces the mutual coupling $b$ ) a novel type of compact elongated mushroom EBG (EM-EBG)structure c) Linear array of four microstrip antenna elements with isolating (EM-EBG) objects

EBG structures are also effective to the design of dual frequency microstrip antennas. It has been proved, in [86], that a properly designed EBG lattice can drive a printed antenna to dual frequency operation, exploiting the higher order modes of the printed element. Generally speaking, a microstrip antenna would be used as dual frequency band radiating system when operates at the fundamental and at one of the higher order resonance frequencies. However, the distributions of the radiated fields, at these two frequencies, are not similar. So, the two bands can not be used for the same type of services. In [86], this problem was confronted integrating the antenna with an EBG surface and dual frequency operation with similar radiation patterns was obtained. The key of this performance is that the antenna works at the two bands with different dielectric layers.

As an application example in[86], results for a 'diamond' antenna consisted of a pair triangular patches were presented(fig. 50). The EBG structure was formed of equilateral triangular microstrip patches.

In figure 51a the variation of the input impedance of the twin triangles without the EBG is illustrated. The triangle patches of the diamond were designed for the $\mathrm{TM}_{10}$ mode at 3.17GHz. At the figure is shown that the second order mode, namely the $\mathrm{TM}_{11}$, appears at $5.6 \mathrm{GHz}$. In figures $51 \mathrm{~b}$ and $51 \mathrm{c}$ the radiation patterns of both modes are depicted. Obviously the patterns are different, whereas the disadvantage of the pattern of $\mathrm{TM}_{11}$, is the deep minimum at the broadside. 


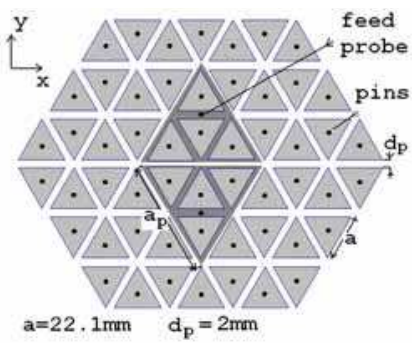

(a)

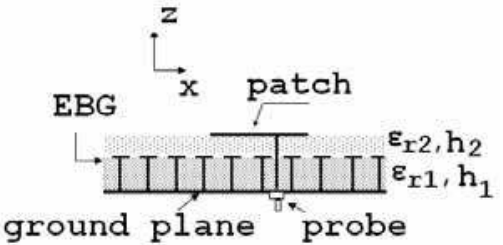

(b)

Fig. 50. Top and side view of a triangular probe fed dual patch antenna over an EBG lattice of triangular printed elements.

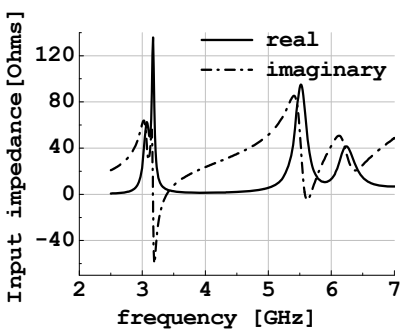

(a)

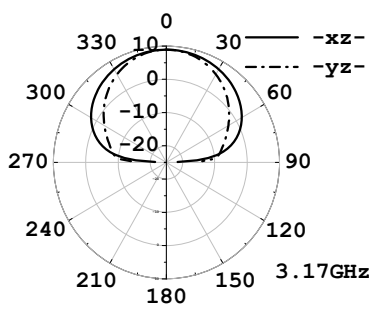

(b)

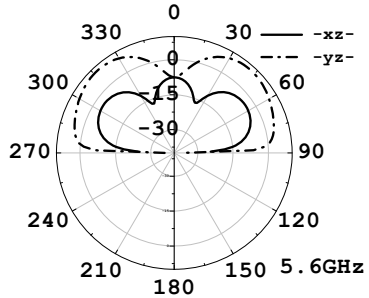

(c)

Fig. 51. (a) Input impedance of the patch antenna of fig 50, without EBG. (b), (c) the xz-and yz-plane directivity patterns of the $\mathrm{TM}_{10}(3.17 \mathrm{GHz})$ and $\mathrm{TM}_{11}(5.6 \mathrm{GHz})$ modes.

In figure 52 the respective results for the diamond antenna integrated with the EBG are presented. The EBG was designed to resonate in a band around $5.6 \mathrm{GHz}$, namely in the band of the second order mode of the diamond. In fig 52a a new resonance appears at $4.3 \mathrm{GHz}$. It is due to the fact that this frequency is out of the EBG band, thereby the EBG performs as an electric conductor, namely as an ordinary metallic grounded sheet. So, the entire structure operates as a microstrip antenna with a single dielectric layer, which is the upper dielectric layer, operating at $\mathrm{TM}_{10}$. It can be also verified using the expressions for the frequency of resonance of a triangular microstrip patch.

The radiation pattern at $4.3 \mathrm{GHz}$ is depicted in fig. $52 \mathrm{c}$ and is a pattern similar to respective ones of microstrip antennas when operate at the fundamental mode. The radiation pattern at $5.6 \mathrm{GHz}$ is depicted in figure $52 \mathrm{~d}$. Around this frequency the EBG is in resonance. Comparing the figures $51 \mathrm{c}$ and $52 \mathrm{~d}$ it is ascertained that the diamond exhibits a different performance in the presence of the EBG. The pattern of $52 \mathrm{~d}$ is similar to that of the fundamental frequency rather, than to that of the ordinary second order mode. It is noted that at both frequency ranges the diamond is driven only by its feeding probes and the matching is satisfactory, as shown in figure 52b. So, the hybrid structure, microstrip diamond-EBG, operates at two frequencies with similar radiation features.

The electric current distribution gives an explanation of the structure's performance. In Fig. $53 \mathrm{a}$ the current at $3.17 \mathrm{GHz}$, namely the $\mathrm{TM}_{10}$ mode of the antenna without the EBG, is shown. This distribution is similar to that of the antenna with the EBG at $4.3 \mathrm{GHz}$ (Fig.53c). 


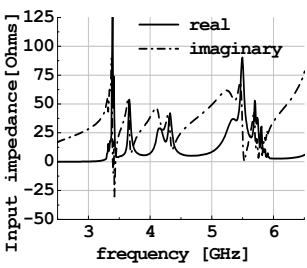

(a)

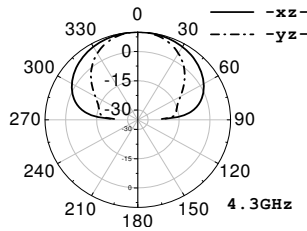

(c)

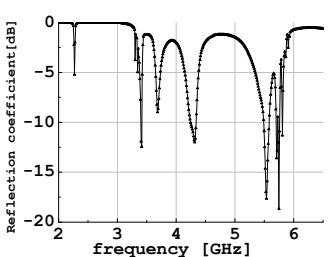

(b)

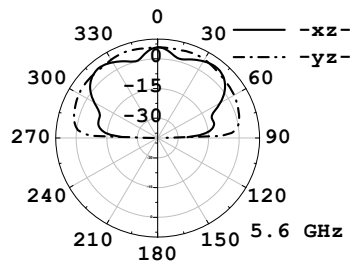

(d)

Fig. 52. (a) Input impedance of the dual triangular patch antenna with EBG (b) The reflection coefficient at the feed ports. (c), (d) the xz-and yz-plane directivity patterns of the $\mathrm{TM}_{10}(4.3 \mathrm{GHz})$ and $\mathrm{TM}_{11}(5.6 \mathrm{GHz})$ modes.

In this case, current does not exist on the EBG surface patches. That's why the patterns of directivity shown in Figures 51b and 52c, are similar. Comparing the current patterns of the $\mathrm{TM}_{11}$ mode without and with EBG(Figs. 53b, 53d), we see that the current distribution is modified. Intense currents flow on the EBG which resonates at this frequency. This performance leads to the field depicted in Fig. 52d.

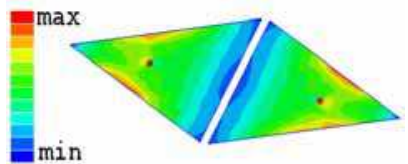

(a)

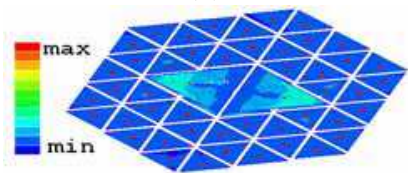

(c)

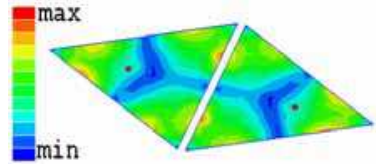

(b)

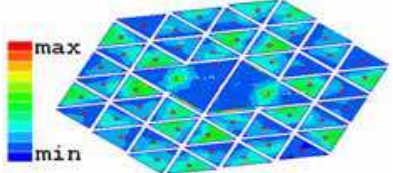

(d)

Fig. 53. (a),(b) Current distribution, at $3.17 \mathrm{GHz}\left(\mathrm{TM}_{10}\right)$ and $5.6 \mathrm{GHz}\left(\mathrm{TM}_{11}\right)$, on the surface of the triangular dual patch antenna without EBG. (c) and (d) The current distribution, at 4.3GHz and $5.6 \mathrm{GHz}$ when the EBG was embedded in the structure. In all cases the phase difference between the probes is $180^{\circ}$.

It is worth to mention that the above described performance would lead to a design procedure for various pairs of operation frequencies and various shapes of antenna and EBG patches. The pair of frequencies have been given, the steps of the design are summarized as follows: 
a. The smaller frequency of the pair is defined as the $f_{10}$ of the radiating patch and the larger one as the frequency $f_{m n}$ of one of the higher modes as well as of the resonance of the EBG.

b. Taking into account that at $\mathrm{f}_{10}$ the EBG will perform as a perfect electric conductor and the patch will work solely with the upper dielectric layer, we select the values of the structural parameters of this layer and calculate the size of the patch.

c. At the selected $\mathrm{f}_{\mathrm{mn}}$ the EBG resonates, so the patch works with both dielectric layers and we determine the characteristics of the lower layer in order the patch, with the already calculated size, to resonate at $\mathrm{f}_{\mathrm{mn}}$ with the double dielectric substrate.

d. We calculate the size of the EBG patches to resonate at $f_{m n}$ in the determined dielectric environment.

For example, suppose that $900 \mathrm{MHz}(\mathrm{GSM})$ and $\sim 2 \mathrm{GHz}(\mathrm{UMTS})$ are the parts of the frequency pair. We set $\mathrm{f}_{10}=900 \mathrm{MHz}$ and $\mathrm{f}_{11}=2 \mathrm{GHz}$. Then selecting for the upper layer, $\varepsilon_{\mathrm{r} 2}=4.4$ and $\mathrm{h}_{2}=0.5 \mathrm{~mm}$, the length of the side of the antenna has to be $\mathrm{a}_{\mathrm{p}}=105 \mathrm{~mm}$, in order to resonate at $900 \mathrm{MHz}$. The calculations are made by equations 19 and 20. In order the patch, with side length $\mathrm{a}_{\mathrm{p}}=105 \mathrm{~mm}$, to resonate at $2 \mathrm{GHz}$ with the $\mathrm{TM}_{11}$ mode, in the presence of the double dielectric layer, the lower layer must have $\varepsilon_{\mathrm{r} 1}=2.2$ and $\mathrm{h}_{1}=1.5 \mathrm{~mm}$. The calculations are made by equations 19, 20 and the expression $\varepsilon_{\mathrm{r}}=\sum_{\mathrm{i}=1}^{2} \mathrm{~h}_{\mathrm{i}} / \sum_{\mathrm{i}=1}^{2}\left(\mathrm{~h}_{\mathrm{i}} / \varepsilon_{\mathrm{ri}}\right)$. The side length of the EBG patch has to be $a=65 \mathrm{~mm}$ in order to resonate at $2 \mathrm{GHz}$, if structured between the determined dielectric layers. These values are of first order approximation. The simulation of the configuration, in virtue of the interaction between the antenna and the EBG, is expected to show that a small perturbation of the size of the elements is necessary to resonate at the pre-specified frequencies.

\section{Stochastic algorithms for antenna design and optimization}

As in the previous sections has been discussed, the design of a printed antenna with advanced characteristics of operation for multi-frequency applications is often a laborious and difficult procedure. To obtain such a performance, the techniques of texturing the surface of the printed patches, the utilization of fractal design procedure or the incorporation of EBG objects are efficient tools for the design, but they are not always enough. Moreover they produce complicated antenna schemes inserting additional parameters that have to be taken into account during the design process. Besides all these, there is a trade off between the antenna's properties which are imposed by the technical requirements. For example, the small size opposes the high gain; the small thickness of the substrate reduces the surface waves and, in this way, the mutual coupling, but also reduces the bandwidth; multi-frequency operation is, in general, not difficult to be achieved but it is not always easy to obtain the frequencies specified by a particular application. On the top of all, there is the difficulty that in most of the cases there are not mathematical expressions in closed form that could help the designer of the antenna. Solution to the above problems would be given by evolutionary techniques, most of which are inspired from the nature and the biology. The Neural Network(NN) method, the Genetic Algorithms(GA), The Particle Swarm Optimization(PSO) are some of the most commonly used methods which were proved efficient in antenna design and more generally in many electromagnetic problems. In the following a brief discussion and a very simple analysis of the NNs and GAs and will be done. The detailed study is beyond the scope of this chapter and would be the content of a separate self-existent book. 


\subsection{Artificial Neural Networks}

A Neural Network is a network of many simple processors connected by communication channels that carry numerical data[87],[88]. The NNs' structure resemble that of the human brain and their operation also imitates the operation of the brain. Two are the basic similarities between them: a)Knowledge is acquired by both through the learning process and b)Interneuron connection strengths, known as synaptic weights, have a small amount, of local memory and are used to store the knowledge. It is exactly the property of the NNs to work as the human brain that make them effective in antenna design. As mentioned above, in an antenna design problem the given data are the technical requirements, for example the gain, the frequency bands of operation etc and what is demanded is the configuration of the antenna. The difficulty in finding the solution of this problem is that the relation between the given and the demanded quantities can not be expressed in closed form and moreover is usually complicated and non linear.

The NN algorithms are ideal for solving problems of the above type, because, working as the human brain, can be trained to find the underlying relation between the values of the antenna parameters, given to them, and the operational features at which these parameters would drive the antenna.

Two are the basic steps when decide to construct and employ a NN: to select the NN's architecture and the strategy of its learning. For all that, it has to be pointed out that it is difficult to know a priori if a specific $\mathrm{NN}$ architecture or learning strategy is suited for a given antenna design problem. So, these selections are substantially a part of the whole problem.

Figure 54 depicts the architecture of a fundamental fully feed-forward NN with Multiple Layer Perceptron Architecture which is commonly used in antenna design.

In this fundamental form the NN consists of three layers. The input layer that includes a M nodes. One (or more) 'hidden' layers with $\mathrm{K}$ nodes and the output layer that includes J nodes. From the mathematical and computer code point of view, the M nodes of the first layer are the positions at which the data $x_{i}, i=1,2 \ldots M$ of the problem will be given. The synapses, or connecting links, between these nodes and those of the hidden layer will transfer the signals, namely the input data, from one layer to the other weighting them by an amount, depending on the link. Each node of the hidden layer gather the accepted signals, adds them and uses this summation as argument of a selected function called 'activation' function. The results are led to the output nodes. The arithmetic values $\mathrm{y}_{\mathrm{j}}$ that appear in the output nodes are substantially the response of the $\mathrm{NN}$ and are the results of the problem under solution. The value of $\mathrm{M}$ is equal to the number of data and $\mathrm{J}$ is equal the required parameters. The number of the hidden layers and their nodes, the type of the activation function, the interconnection, or not, of all the nodes are part of the study of the NN.

In the case of the design of a rectangular printed antenna, for example, if the technical requirements are a specific value of gain and the resonance frequency, two input nodes are necessary. The output nodes would potentially be four, giving the values of the lengths of the sides of the patch the dielectric constant and the height of the substrate.

In order to receive from the $\mathrm{NN}$ the correct response it must be trained at first. The training is the fundamental procedure of a NN and the first step of this procedure is the construction of a training set. It has to contain a number $\mathrm{N}_{t}$ of pairs of vectors. If , for example we suppose that $x_{i}, i=1,2 \ldots M$ describe the parameters of operation(gain, bandwidth, etc) and $y_{i}, i=1,2 \ldots J$ stand for the geometry and material parameters of the antenna, the first part 


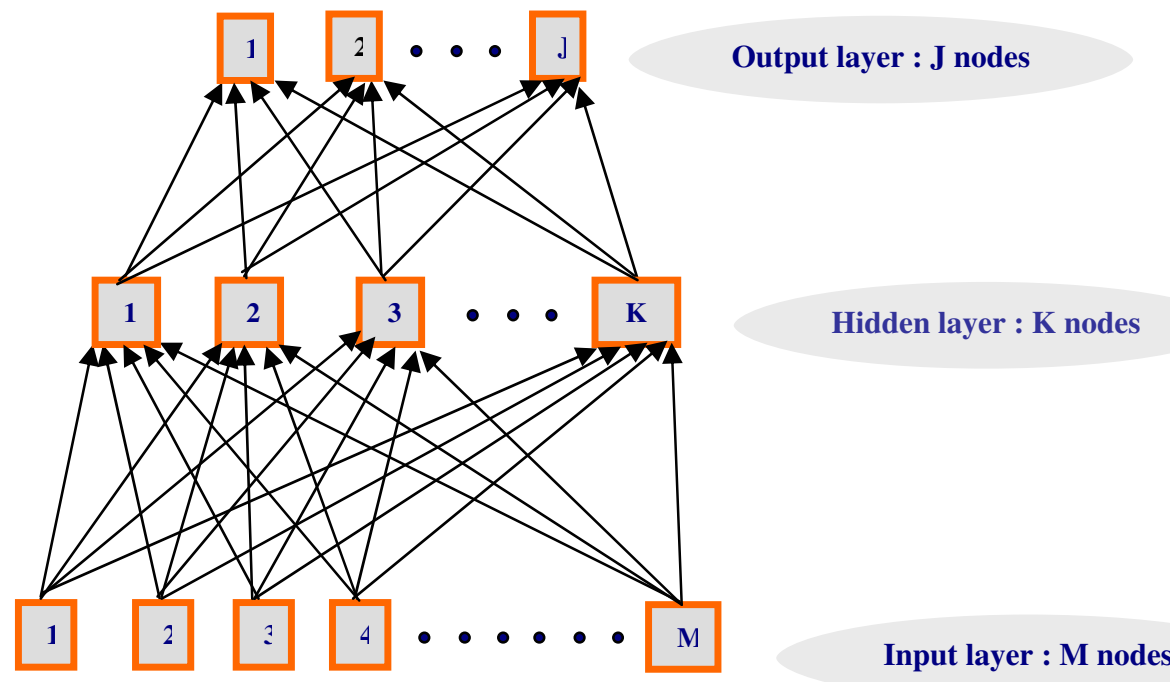

Fig. 54. The basic fully feed forward NN with Multiple Layer Perceptron Architecture

of the pair is the input vector containing the values of the input parameters $x_{i}$, whereas the second part of the pair is a vector with the $y_{i}$ values, that result from the $x_{i}$ and the functions that relate all $x_{i}$ and $y_{i}$. Exactly these functions, which are unknown, are the cause we turn to the employment of NNs techniques. A well trained NN would successfully make the mapping from the $x_{i}$ parameters to the $y_{i}$ results, thus finding the relation between them without be necessary to find the mathematical function which connects them. In the cases of antenna design the training set can be created simulating the performance of $\mathrm{N}_{t}$ antennas with $\mathrm{N}_{t}$ different combinations of parameter values $\mathrm{y}_{\mathrm{i}}$ and recording the corresponding $\mathrm{x}_{\mathrm{i}}$ values.

The training of the NN is made by inserting at its input repetitively and in random order, the input vectors of the training collection and simultaneously presenting at its output nodes, the respective output vector. If the actual NN output does not match the respective output then the $\mathrm{NN}^{\prime} \mathrm{S}$ interconnection weights are modified to move the actual output closer to the desired output. The criterion of this matching is an error function defined as

$$
E_{p}(w)=\frac{1}{2} \sum_{i=1}^{J}\left[d_{j}(p)-y_{j}^{o}(p)\right]^{2}
$$

where $d_{j}(p)$ is the value at the $j^{\text {th }}$ output node, expected in accordance with the $j^{\text {th }}$ input element of the $p^{\text {th }}$ training set, and $y_{j}^{o}(p)$ is the value of the node calculated by the the NN with a specific set $\mathrm{w}$ of weights.

The above process is repeated for all the input -output pairs of the train collection until we reach a point, that is a set of proper weights, where the actual output of the NN for every input of the collection matches to our satisfaction the desired output, namely the minimization of the function $\mathrm{E}_{\mathrm{p}}(\mathrm{w})$. Then, it is considered that the learning process is over and that the trained NN has successfully approximated the desired mapping between the inputs and the corresponding outputs of the collection and in this way has found out the 
non linear relation between the configuration parameters $y_{i}$ of the antenna and its operational features. To test the effectiveness of the NN, it is exposed in a collection of input-output vectors which were not included in the training set. If the results are satisfactory the $\mathrm{NN}$ is ready for generalization. In other words we can give to its input a set of values of desired properties of operation and the well trained $\mathrm{NN}$ will give us the parameter values to construct the antenna that will have these properties.

The described learning strategy is characterized as 'supervised'. The main feature of the process is that the NN 'knows' externally the expected output vector for each pair of vectors it accepts during the training. So, it construct an error quantity defined as the difference between the actual response of the network and the desired response of the network and the $\mathrm{NN}$ is trained trying to minimize the error quantity. Alternate categories of learning are the unsupervised and the hybrid learning. In unsupervised or self-organized learning, we can not provide the $\mathrm{NN}$ with a desired or target response for each training vector it accepts at its input, because it is not known. Instead, a provision is made to identify a measure of the quality of the representation that the network is required to learn and the free parameters of the network are optimized with respect to that measure. On the other hand, there are problems at which a purely supervised learning procedure is not very efficient or many input-output training pairs have to be used. In the last case the difficulty is that as the training data set increases in size the training time of the multilayer perceptron increases exponentially. A way of dealing with this problem is to incorporate unsupervised and supervised learning. This is what we call hybrid learning. In this approach we first group the data into a number of categories which are a lot fewer than the number of input-output pairs in the original training set. It is accomplished by using an appropriate unsupervised learning algorithm. Having reduced the training data set significantly, we can subsequently utilize a supervised learning NN architecture.

In many applications of $\mathrm{NN}$ in microstrip antenna design, the training vectors include the parameters of the geometry of the antenna and those of the substrate and the required results at the output of the NN are the indices of operation, that is the gain, the resonant frequency, the bandwidth, the polarization etc,[89], or the radiation pattern[90]. In [40], however, the inverse problem is solved. Vectors of the sampled radiation pattern are used for the training of the $\mathrm{NN}$, whereas the structural parameters of the antenna are provided by the NN at the output nodes. By the constructed NN, the synthesis of a dual band, multilayered printed antenna, textured with slits was obtained. The NN methodology has also been used successfully in the synthesis of EBG surfaces with specific reflection phase characteristics[91], suitable for low profile polarization diversity antenna systems.

It is worth noting that the solution given by the NN is not necessarily the optimum. This fact is due to the nature of the NN algorithms. A NN gives an answer that fits to the imposed requirements which has accepted, in the form of data, and if it is well trained the results fulfils the requirements. In many applications it is enough. What would be done in the case in which the prescriptions are imposed in the form of constraints? For example suppose to design an antenna with gain value greater, as possible, than a specific value and Side Lobe Level lower, as possible, than a pre-defined value. This type of specifications have the meaning of optimization and techniques with a different nature would be used. An ordinary efficient method for this purpose is a Genetic Algorithm.

\subsection{Genetic Algorithms(GA)}

Optimization is generally the process of making something better. The process consists in making variations on an initial concept and using the gained information to improve the 
ideas. From the application side of view, optimization is the process of adjusting the inputs or the characteristics of a device or generally a creation, using a mathematical or experimental process in order to find the minimum or maximum output results. In antenna applications the scope of an optimization procedure is to find out not just a solution but the best one for a specific application. The terminology of 'best' solution implies that a)there is more than one solutions and b) the solutions are not of equal value. The definition of 'best' is relative to the application, namely the technical specifications imposed, and the tolerance allowed.

One of the most commonly techniques for antenna optimization is the employment of Genetic Algorithms[92]-[94]. GAs model natural selection and evolution using biological processes to optimize highly complex functions. The method was developed by John Holland, in 1975, and finally popularized by one of his students David Goldberg, in 1989.

Genetic Algorithms have been widely used in antenna synthesis and optimization problems. They have been proved efficient in finding the proper excitations or the suitable division of the arrays in sub-arrays in order the antenna to produce a desired radiation pattern with respect to the SLL and the directions of the nulls, to optimize the antenna by broadening the frequency bandwidth or in thinning an array the target being, in all cases, the achievement of special performance features. GAs are also a frequent choice for the design and optimization of microstrip antennas, especially broadband, slit textured, fractal printed, or not, as well as optimized EBG antennas with enhanced gain [95]-[98].

Several antenna optimization problems are characterized multi-dimensional. It means that there are more than one parameters that are taken into account for the evaluation of their performance. These parameters are ordered in a vector $\bar{p}=\left[p_{1}, p_{2}, \ldots \ldots . p_{N}\right]$, where $N$ is the number of the parameters, termed chromosome. At the same time the problem would be multi-objective[97], if the indices of performance are more than one; for example, the gain, the SLL, bandwidth etc. These indices are functions, defined as $g(\cdot)$, of the parameters of the problem, termed objective functions. At each chromosome $\bar{p}$, a set of values of these functions corresponds, making a vector $g_{C}(\bar{p})=\left[g_{1}(\bar{p})-c_{1}, g_{2}(\bar{p})-c_{2}, \ldots \ldots . . g_{M}(\bar{p})-c_{M}\right]$ where $M$ is the number of the functions-indices and $c_{i}$ is a constrained value for the respective function. Vector $\mathrm{g}_{\mathrm{C}}(\overline{\mathrm{p}})$ is termed, cost or fitness vector and these constrained values are imposed by the requirements of the problem. The aim of the GA is to minimize the cost vector.

The general steps of a GA are the following:

Step 1. Generation of an initial population of $\mathrm{N}_{\text {pop }}^{\text {in }}$ chromosomes $\overline{\mathrm{p}}$ applying a procedure of random selection. This population, termed generation, does not remain constant but changes gradually in the following steps.

Step 2. Calculation of the cost vectors for all chromosomes, $\mathrm{g}_{\mathrm{ci}}\left(\overline{\mathrm{p}}^{\mathrm{i}}\right), \quad \mathrm{i}=1,2, \ldots \mathrm{N}_{\text {pop }}^{1}$

Step 3. From this population only a number of members is kept for the next generation whereas the rest are discarded. The selection of the members which will survive is made by pre-specified constraints expressed by qualities or inequalities. The chromosomes which survive undergo the process of mating: Pairs of chromosomes are selected and then are used as parents for the generation, via pre-specified rules, of a new pair of offspring. In these rules, steps of randomness are incorporated. The total of the $\mathrm{Ni}$ offspring produced in this way constitute a new generation.

Step 4. The cost vectors $g_{c i}\left(\bar{p}^{i}\right)$ for all $i=1,2 \ldots . . N i$ chromosomes are calculated. 
Step 5. Using, potentially, a threshold value for each component of each cost vector, or for the total of them, we select a subtotal of the $\mathrm{N}^{\mathrm{i}}$ chromosomes which will be the population of the next generation. The thresholds may be not constant and the new generation includes the individuals that correspond to all the $g_{c i}\left(\bar{p}^{i}\right)$ which passed the test of thresholding and at the same time ensure the feasibility of the antenna. In this way the number chromosomes in the populations gradually decrease and better performers are included from generation to generation.

Step 6. The process goes back to step 3 for the mating of the members of the new generation. This procedure is repeated iteratively, either a pre-specified number of times or until all the chromosomes of the population fulfill a pre-defined criterion.

Additional steps as the mutation, which is a separate process to change the chromosomes or/and elitism strategies can be incorporated in the GA depending on the problem. Elitism strategies give solution to the following problem: during the process of a simple genetic algorithm it is possible for the next generation to have a best individual with a lower fitness, with concern to the cost function, than the best individual encountered in a preceding generation. This loss of the best individual occurs due to the probabilistic nature of the GA selection and mutation. A simple test can be added to verify that the best individual in the new generation is at least as good as the one from the preceding generation. Saving and inserting the best individuals from the last generation is known as an elitist action. Elitism can be used to ensure that there is a monotonic increase in the best fitness in the population as a function of time during the GA process.

The basic characteristics and the advantages of the GAs are summarized as follows:

1. The GAs can be used in problems with one or more objective functions. In these cases of more than one optimum solutions would be produced.

2. Constraints, which ensure the feasibility of the antenna solutions and the their compliance the technical specifications can be incorporated.

3. In GAs the population from one generation to the next changes through mutation and perhaps additional constrains imposed by the problem under solution. In this way, the space of possible solutions is explored more efficiently and the GA, looking simultaneously for more than one minima, can give a final solution, which in the case of one objective function, would be not simply a local optimum but a global optimum.

4. The GAs start with a population of feasible solutions instead of a single one(as in more traditional approaches)

5. Gas use probabilistic transition and selection rules, not deterministic rules.

6. GAs optimize with continuous or discrete parameters

7. A GA doesn' $t$ require derivative information

8. GAs deal with a large number of parameters

9. GAs optimize parameters with extremely complex cost surfaces because they can jump out of a local minimum

10. Works with numerically generated data, experimental data or analytical functions

It has to be pointed out that, as in the case of NNs, it is difficult to know a priori if a specific GA is the proper for a given antenna design problem or how to improve the GA when it fails. So, an extended study is often necessary and the study concerns not only the specific antenna problem but the GA itself. 


\section{References}

[1] R. Chair, K.M. Luk, and K.F. Lee, ' Miniature multilayer shorted patch antenna,' Electron. Lett., Vol. 36, pp. 3-4, Jan. 6, 2000

[2] L. Zaid, G. Kossiavas, J. Dauvignac, J. Cazajous, and A. Papiernik, 'Dual-frequency and broad-band antennas with stacked quarter wavelength elements,' IEEE Trans. Antennas Propagat., Vol. 47, pp. 654-660, April 1999

[3] Kin-Lu Wong, 'Compact and broadband microstrip antennas', John Wiley \& Sons, New York, 2002.

[4] C.K. Wu and K.L. Wong, 'Broadband microstrip antenna with directly coupled and gap coupled parasitic patches', Microwave Opt. Technol. Lett., Vol. 22, pp. 348-349, Sept. 5,1999

[5] J.Y. Sze and K.L. Wong, 'Broadband rectangular microstrip antenna with a pair of toothbrush-shaped slots', Electron Lett., Vol. 34, pp. 2186-2187, Nov. 12, 1998.

[6] J.Y. Sze and K.L. Wong, 'Single-layer single -patch broadband rectangular microstrip antenna,' Microwave Opt. Technol. Lett. , Vol. 22, pp. 234-236, Aug. 20, 1999.

[7] C.-L. Li, H-,H. Wang, H.-J.Lin, X-.E. Shi, W.-T. Li and L. Xu, 'Analysis and design of broadband microstrip patch antenna with a pair of double cross-shaped slots', Proc. Of International conference on microwave and millimeter wave technology(ICMMT), 2010.

[8] T. Huynh and K. F. Lee , 'Single-layer single-patch wideband microstrip antenna,' Electron. Lett. , Vol. 31, pp. 1310-1311, Aug. 3, 1995

[9] K.L. Wong and W.H. Hsu, 'Broadband triangular microstrip antenna with U-shaped slot', Electron. Lett. , vol. 33, pp. 2085-2087, Dec. 4, 1997

[10] S. Weigand, G.H. Huff, K.H. Pan, and J.T. Bernhard, 'Analysis and design of broadband single-layer rectangular U-Slot microstrip patch antenna,' IEEE Transactions on Antennas and Propagation, AP-51, 3, pp. 457-468, 2003.

[11] K.F. Tong and T.P. Wong, 'Circularly polarized U-slot antenna', IEEE Transactions on Antennas and Propagation, AP-55, 8, pp. 2382-2385, 2007

[12] K.F. Lee, S.L. Steven Yang, A. A. Kishk, 'Dual and multiband U-slot patch antennas', IEEE Antennas and Wireless Propagation Letters, Vol. 7, pp. 645-647, 2008

[13] H. Wang, X.B. Huang and D.G. Fang, 'A single layer wideband U-slot microstrip patch antenna array,' IEEE Antennas and Wireless Propagation Letters, Vol. 7, pp. 9-12, 2008

[14] Lingjian Li and Zhirum Hu, 'A wideband linear U-slot microstrip patch antenna array for wireless applications', International eburnal of Electronics, Vol. 96, 7, pp. 755-765, 2009

[15] Kai Fong Lee, Shing Lung , Steven Yang, Ahmed A. Kishk and Kwai Man Luk, 'The versatile U-slot patch antennas,' IEEE Antennas and Propagation Magazine, Vol. 52, 1, pp. 71-88, 2010.

[16] K.F. Tong, K.M. Luk, K.F. Lee and R.Q. Lee, 'A broadband U-slot rectangular patch antenna on a microwave substrate', IEEE Transactions on Antennas and Propagation, AP-48, 6, 954-969, 2000.

[17] R. Chair, K.F. Lee, C.L. Mak, K.M. Lukand A.A. Kishk, 'Miniature wideband half U-slot and half E-Shaped patch', IEEE Transactions on Antennas and Propagation, AP-53, vol. 8, pp. 2645-2652, 2005. 
[18] A.A. Deshmukh and K.P. Ray, 'Compact broadband slotted rectangular microstrip antenna', IEEE Antennas and Wireless Propagation Letters, Vol. 8, pp. 1410-1413, 2009.

[19] Y.X. Guo, A. Shackelford, K. F. Lee and K.M. Luk, 'Broadband quarter-patch antenna with a U-shaped slot', Microwave Opt. Technol. Lett. , Vol. 28, pp. 328-330, 2001

[20] A. Shackelford, K.F. Lee and K.M. Luk, 'Design of small-size wid-bandwidth microstrip patch antennas', IEEE Antennas and Propagation Magazine, Vol. 45, 1, pp. 75-83, 2003.

[21] C.L. Mak, R. Chair, K.F. lee, K.M. Luk AND a.a. Kishk, 'Half U-slot patch antenna with shorting wall', Electron. Lett. , Vol. 39, 1779-1780, 2003.

[22] K.L. Wong and W.H. Hsu, 'A broadband rectangular patch antenna with a pair of wide slits", IEEE Trans. Antennas and Propagation, Vol. 49, 9, pp. 1345-1347, 2001.

[23] W.H. Hsu and K.L. Wong, 'A wideband circular patch antenna', Microwave Opt. Technol. Lett., Vol. 25, pp. 327-328, June 5, 2000

[24] K.M. Pramod, R. Jyoti, S. S. Kumar, V.S.K. Reddy, 'Simplified and efficient technique for designing of broadband patch antenna,' Proc. of Applied Electromagnetics Conference (AEMC), 2009

[25] L. Peng, C. Ruan, Y. Zhang, 'A novel compact broadband microstrip antenna,' Proc. Asia-Pasific Microwave Conference(APMC2007), 2007

[26] Y. Chen, S. Yang and Z. Nie, ' Bandwidth enhancement method for low profile Eshaped microstrip patch antennas,' IEEE Trans. Antennas and Propagation, Vol. 58, 7, pp. 2442-2447, 2010.

[27] K.M. Pramod, S. S. Kumar, R. Jyoti, V.S.K. Reddy, and P.N.S. Rao, 'Novel structural design for compact and broadband patch antenna,' International Workshop on Antenna Technology (IWAT), 2010.

[28] S. Xiao, Z. Shao, B.Z. Wang, M.T. Zhou and M. Fujise, 'Design of low profile microstrip antenna with enhanced bandwidth and reduced size,' IEEE Trans. Antennas and Propagation, Vol. 54, 5, pp. 1594-1599, 2006.

[29] S. Zhaohui, L. Meijia and D. Zhiyong, ' An improved design of microstrip Archimedeam spiral antenna', Proc. of International Conference on Microwave and millimeterWave Technology(ICMMT), 2007

[30] L. Bian and X.-Q. Che, 'Application of the equivalent Circuit Method in Designing the Vivaldi UWB antenna', $4^{\text {th }}$ International Conference on Wireless Communications, Networking and Mobile Computing(WiCOM '08), 2008.

[31] X. Zhuge and A. Yarovoy, ' Design of low profile antipodal Vivaldi antenna for ultrawideband near field imaging', Proc. of the Fourth European Conference on Antennas and Propagation(EuCAP), 2010.

[32] J. George, M. Depukumar, C.K. Aanandan, P.Mohanan and K.G. Nair, 'New compact microstrip antenna', Electron Let. , Vol. 32, pp.508-509, 1996.

[33] A.A. Eldek, A.Z. Elsherbeni and C. E. Smith, 'Wide-band modified printed bow-tie antenna with single and dual polarization for C- and X-Band applications', IEEE Transactions on Antennas and Propagation, Vol. 53, no. 9, pp. 3067-3072, 2005

[34] T. Karacolak and E. Topsakal, 'A double-sided rounded bow-tie antenna (DSRBA) for UWB communication', IEEE Antennas and Wireless Proagation Letters, Vol. 5, pp. 446-449, 2006 
[35] Z. D. Liu, P.S. Hall and D. Wake 'Dual-frequency planar inverted F antenna', IEEE Trans. Antennas and Propagation, Vol. 45, 10, pp.1451-1458, 1997.

[36] P. Salonen, M. Keskilammi and M. Kivikoski, 'New slot configurations for dual-band planar inverted -F antenna', Microwave Opt. Technol. Lett., Vol. 28, No. 5, pp. 293298, 2001

[37] M. Martinez-Vasquez, M. Geissler, D. Heberling, A. Martinez-Gonzalez and D. SanchezHernandez, 'Compact dual-band antenna for mobile handsets', Microwave Opt. Technol. Lett. , Vol. 32, No. 2, pp.87-88, 2002

[38] M. Polívka, 'Multiband behavior of the rectangular microstrip patch antenna modified by T-notch perturbation elements', Proc. Of $18^{\text {th }}$ International Conference on Applied Electromagnetics and Communications(ICECom), 2005

[39] I. Sarkar, P.P Sarkar and S.K. Chowdhury, 'A novel compact, microstrip antenna with multifrequency operation', International Seminar/Workshop on Direct and Inverse Problems of Electromagnetic and Acoustic Wave Theory(DIPED 2009), 2009

[40] K. Siakavara , 'Artificial Neural Network Based Design of a Three-Layered Microstrip Circular Ring Antenna with Specified Multi-Frequency Operation', Neural Computing and Applications, Vol. 18, pp. 57-64, 2009

[41] A. Manassas, T. Kaifas, K. Siakavara, 'Multiband printed antenna for low frequenies WLAN applications', International eburnal of Microwawe and Optical Technology, Vol 2, No 3, pp. 182-186, 2007

[42] K. Siakavara and T. Ganatsos, 'A compact quadruple crossed dipole microstrip antenna design for dual frequency and beam scanning operation', Electrical Engineering, Vol. 86, pp. 117-119, 2004

[43] C.A. Balanis, Advanced Engineering Electromagnetics, John Wiley \& Sons, New York, 1989

[44] D. M. Kokotoff, J.T. Aberle and R.B. Waterhouse, 'Rigorous analysis of probe fed printed annular ring antennas', IEEE Trans. on Antennas and Propagation, Vol. 47, No. 2, pp.384-388, Feb. 1999.

[45] R. Garg, P. Bhartia, I. Bahl,, A. Ittipiboon, Microstrip Antenna Design Handbook, Artech House, Boston-London, 2001

[46] Y. Tawk, K.Y. Kabalan, A. El-Hajj, C.G. Christodoulou and J. Constantine, 'A simple multiband printed bowtie antenna', IEEE Antennas and Wireless Propagation Letters, Vol. 7, pp. 557-560, 2008

[47] H. K. Kan and R.B. Waterhouse, 'Shorted spiral-like printed antennas', IEEE Trans. on Antennas and Propagation, Vol. 50, No. 3, pp. 396-397, 2002.

[48] JoongHan Yoon, 'Fabrication and measurement of modified spiral-patch antenna for use as a triple-band antenna', Microwawe Opt. Technol. Lett., Vol. 48, No 7, pp.12751279, 2006

[49] J. Anguera, G. Font, C. Puente, C. Borjia and J. Soler, 'Multifrequency microstrip patch antenna using multiple stacked elements', IEEE Microwave and Wireless Components Letters, Vol. 13, no 3, pp.123-124, 2003

[50] D. H. Werner and S. Ganguly, 'An Overview of Fractal Antenna Engineering Research', IEEE Antennas and Propagation Magazine, Vol. 45, No 1, pp. 38-57, 2003 
[51] J. P. Gianvittorio and Y. Rahmat-Samii, 'Fractal antennas: A novel antenna miniaturization technique and applications', IEEE Antennas and Propagation Magazine, Vol. 44, No. 1, pp. 20-36, 2002.

[52] C. A. Balanis, Antenna Theory_Analysis and Design. John Wiley \& Sons, New Jersey, third edition, 2005.

[53] E. -O Peitgen and D. Saupe, The science of fractal images, Springer-Verlag, Berlin/ New York, 1988

[54] D.H. Werner, R.L. Haupt, and P.L. Werner, "Fractal Antenna Engineering: The Theory and Design of Fractal Antenna Arrays", IEEE Antennas and Propagation Magazine, Vol. 41, No 5, 37-59, 1999.

[55] K. Siakavara, E. Vafiadis, and J.N. Sahalos, "On the Design of a Direct Radiating Array by Using the Fractal Technique," Proc. of the 3rd European Conference on Antennas and Propagation, pp. 1242-1246, Berlin, Germany 23-27, March 2009

[56] K. Siakavara, 'Novel fractal antenna arrays for Satellite Networks: Circular ring Sierpinski carpet arrays optimized by Genetic Algorithms', Progress in Electromagnetic Research_PIER, Vol. 103, pp. 115-138, 2010.

[57] K. Siakavara, 'Hybrid-Fractal Direct Radiating Antenna Arrays with Small Number of Elements for Satellite Communications', IEEE Trans. on Antennas and Propagation, Vol. 58, No 6, pp. 2102-2106, June 2010.

[58] C. Borja and J. Romeu, ' On the behavior of Koch island fractal boundary microstrip antenna', IEEE Transactions on Antennas and Propagation, Vol. 51, pp. 1281-1291, 2003.

[59] M. Bledowski and M. Kitlinski, 'Compact-planar monopole loop antennas for 802.11b/g WLAN systems', Microwave and Optical Technology Letters, Vol. 49, No 9, pp. 22992303, 2007.

[60] K. Siakavara, 'A compact fractal microstrip antenna for GPS and terrestrial radio services,' International eburnal of Electronics, Vol. 94, No. 3, pp. 277-283, March 2007

[61] C. Puente-Baliarda, J. Romeue, R. Pous and A. Cardana, 'On the behavior of the Sierpinski multiband fractal antenna', IEEE Transactions on Antennas and Propagation, Vol. 46, No. 4, p. 518-523, 1998.

[62] S. R. Best, ' On the significance of self-similar fractal geometry in determining the multiband behavior of the Sierpinski gasket antenna', IEEE Antennas and Wireless Propagation Letters, Vol. 1, p. 22-25, 2002.

[63] Y. Na and S. Xiao-Wei, 'Analysis of the multiband behavior on Sierpinski carpet fractal antennas', Proc. of Asia Pasific Conference-Microwave Conference, 2005

[64] C. Puente Baliarda, C. Borja Borau, M. Navarro Rodero and J. Romeu, 'An iterative model for fractal antennas: application to the Sierpinski gasket antenna', IEEE Transactions on Antennas and Propagation, Vol. 48, No. 5, pp. 713-719, 2000.

[65] R. K. Mishra, R. Ghatak and D.R. Poddar, ‘Design formula for Sierpinski Gasket, prefractal planar -monopole antennas', IEEE Antennas and Propagation Magazine, Vol. 50, No. 3, pp.104-107, 2008

[66] G.F. Tsachtsiris, C.F. Soras, and M.P. Karaboikis, and V.T. Makios, 'Analysis of a modified Sierpinski gasket monopole antenna printed on dual band wireless devices', IEEE Transactions on Antennas and Propagation, Vol. 52, pp. 2571-2579, 2004 
[67] M.P. Salmasi, F. Hodjatkashani, and M. N. Azarmanesh, “A Novel Broadband Fractal Sierpinski Shaped, Microstrip Antenna” , Progress In Electromagnetics Research, PIER C 4, pp. 179-190, 2008.

[68] K. Zhang, Ch. Chen, Ch. Guo, J. Xu, ' On the behavior of conformal Sierpinski fractal microstrip antenna', Proc. of International conference on Microwave and Millileter Wave Technology(ICMMT), pp. 1073-1076, 2008.

[69] J. Yeo, and R. Mittra, 'Design of conformal multiband antennas based on fractal concepts', Microwawe and Optical Technology Letters, Vol. 36, pp. 333-338, 2003.

[70] K. J. Vinoy, K.A. Jose, V.K. Varadan and V.V. Varadan, ' Resonant frequency of Hilbert curve fractal antennas', Proc. of IEEE Antennas and Propagation International symposium, pp. 648-651, 2001.

[71] K. Wang, Z. Wang, X. Lei, M. Zhou, ,Resonant frequency and sensitivity analysis of Hilbert curve fractal antennas for DRM communications', Proc. of 8th International Symposium on Antennas, Propagation and EM Theory(ISAPE 2008), pp. 137-139, 2008.

[72] D. Gala, J. Soler, C. Puente, C. Borja and J. Anguera, 'Miniature microstrip patch antenna loaded with a space-filling transmission line based on the fractal Hilbert curve', Microwawe and Optical Technology Letters, Vol. 38, No. 4, pp. 311-312, 2003.

[73] G.S.A. Shaker and S. Safavi-Naeini, 'Highly miniaturized ractal antennas', Proc. of IEEE Radio and Wireless Symposium, pp. 125-128, 2007.

[74] J.-C. Liu, B.-H. Zeng, H.L. Chen, S.-S. Bore And D.-C. Chang, 'Compact fractal antenna with self-complimentary Hilbert-curves for WLAN dual-band and circular polarization applications', Microwave and Optical Technology Letters, Vol. 5, No. 11, pp. 2535-2539, 2010.

[75] K. Siakavara and F. Tsaldaris , "A Multi-wideband Microstrip Antenna Designed by the Square Curve Fractal Technique," Microwave and Optical Technology Letters, Vol. 41, No 3, pp180-185, 2004.

[76] D. Sievenpiper, 1. Zhang, R.F. Jimenez Broas , N.G. Alexopoulos , E. Yablonovitch , 'High impedance electromagnetic surfaces with a forbidden frequency band,' IEEE Trans. Microwave Theory and Techniques, Vol. 47, No 11, pp. 2059-2074, Nov. 1999.

[77] Fan Yang and Yahya Rahmat-Samii, Electromagnetic Band Gap Structures in Antenna Engineering, Cambridge University Press, 2009.

[78] R. Collin, Field theory of guided waves , 2nd ed. New York , IEEE PRESS 1991

[79] N. Engheta, R.W. Ziolkowski, Metamaterials-Physics and Engineering Extrapolations, IEEE Press and John Wiley \& Sons, 2006.

[80] S. A. Tretyakov and C.R. Simovski, 'Dynamic model for artificial reactive impedance surfaces', I of Electromagnetic waves and applications, Vol. 17, No. 1, pp. 131-145, 2003

[81] F. Yang and Y. Rahmat-Samii, 'Reflection phase characterizations of the EBG ground plane for low profile wire antenna applications', IEEE Transactions on Antennas and Propagation, Vol. 52, No. 10, pp. 2691-2703, 2003.

[82] L. Akhooondzadeh-Asl, D. J. Kern, P. S. Hall and D. H. Werner, 'Wideband dipoles on Electromagnetic bandgap ground planes', IEEE Transactions on Antennas and Propagation, Vol. 55, No. 9, pp. 2426-2434, 2007 
[83] F. Yang and Y. Rahmat-Samii, 'Microstrip antennas integrated with electromagnetic band-gap(EBG) structures: a low mutual coupling design for array applications', IEEE Transactions on Antennas and Propagation, Vol. 51, No. 10, pp. 2936-2946, 2003.

[84] E. Rajo-Iglesias, O. Quevedo-Teruel and L. Inclan-Sanchez, 'Mutual coupling reduction in patch antenna arrays by using a planar EBG structure and a multilayer dielectric substrate', IEEE Transactions on Antennas and Propagation, Vol. 56, No. 6, pp. 16481655, 2008.

[85] M. Coulombe, S. F. Koodiani and Ch. Caloz, 'Compact enlogated mushroom (EM)-EBG structure for enhancement of patch antenna array performance', IEEE Transactions on Antennas and Propagation, Vol. 58, No. 4, pp. 1076-1086, 2010.

[86] K. Siakavara , T. Ganatsos, 'Modification of the Radiation Patterns of Higher Order Modes of Triangular Printed Antennas by EBG Ground Planes,' IEEE Antennas and Wireless Propagation Letters, Vol. 8 , pp. 124-128, 2009

[87] S. Haykin, Neural networks - A comprehensive foundation, (Second edition),Prentice Hall Inc. , Boston, 1999.

[88] C. Christodoulou and M. Georgiopoulos, Applications of neural networks in electromagnetics, Artech House, Boston, 2001.

[89] S. Lebbar, Z. Guennoun, M. Drissi and F. Riouch, 'A compact and broadband microstrip antenna design using a geometrical-methodology based artificial neural network', IEEE Antennas and Propagation Magazine, Vol. 48, No 2, pp.146-154, 2006

[90] D.K. Neog, S.S. Pattnaik, D. C. Panda, S. Devi, B. Khuntia and M. Dutta, 'Design of a wideband microstrip antenna and the use of artificial neural networks in parameters calculation', IEEE Antennas and Propagation Magazine, Vol. 47, No 3, pp. 60-65, 2005

[91] T. Ganatsos, K. Siakavara and J.N. Sahalos, ‘Neural Network - Based Design of EBG Surfaces for Effective Polarization Diversity of Wireless Communications Antenna Systems', PIERS On-line, Vol. 3, No 8, pp. 1165-1169, 2007

[92] Haupt, R. and S.E. Haupt, Practical Genetic Algorithms, John Wiley \& Sons, 1998.

[93] D.E. Goldberg, Genetic Algorithms in Search Optimization \&Machine Learning, Addison Wesley Longman,1989

[94] Y. Rahmat-Samii and E. Michielssen, Electromagnetic Optimization by Genetic Algorithms, Wiley , New York, 1999

[95] R. K. Shaw and D.H. Werner, 'Design of optimal broadband microstrip antenna elements in the array environment using genetic algorithms', Proc. of IEEE Antennas and Propagation Society International Symposium, pp. 3727-3730, 2006

[96] R. Ghatak, D.R. Poddar, R.K. Mishra, 'Design of Sierpinski gasket fractal microstrip antenna using real coded genetic algorithm', Microwawes, Antenna \& Propagation, IET, Vol. 3, Issue 7, pp. 1133-1140, 2009

[97] K. Siakavara, 'Novel Fractal Antenna Arrays for Satellite Networks: Circular Ring Sierpinski Carpet Arrays Optimized by Genetic Algorithms', Progress In Electromagnetics Research, Vol. 103, pp. 115-138, 2010. 
[98] Y. Ge, K.,P. Esselle, Y. Hao, 'Design of low-profile high-gain EBG resonator antennas using genetic algorithm' , IEEE Antennas and Wireless Propagation Letters, Vo. 6, pp. 480-483, 2007 


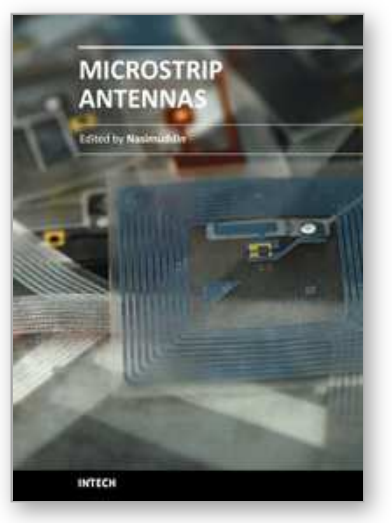

\author{
Microstrip Antennas \\ Edited by Prof. Nasimuddin Nasimuddin
}

ISBN 978-953-307-247-0

Hard cover, 540 pages

Publisher InTech

Published online 04, April, 2011

Published in print edition April, 2011

In the last 40 years, the microstrip antenna has been developed for many communication systems such as radars, sensors, wireless, satellite, broadcasting, ultra-wideband, radio frequency identifications (RFIDs), reader devices etc. The progress in modern wireless communication systems has dramatically increased the demand for microstrip antennas. In this book some recent advances in microstrip antennas are presented.

\title{
How to reference
}

In order to correctly reference this scholarly work, feel free to copy and paste the following:

K. Siakavara (2011). Methods to Design Microstrip Antennas for Modern Applications, Microstrip Antennas, Prof. Nasimuddin Nasimuddin (Ed.), ISBN: 978-953-307-247-0, InTech, Available from:

http://www.intechopen.com/books/microstrip-antennas/methods-to-design-microstrip-antennas-for-modernapplications

\section{INTECH}

open science | open minds

\author{
InTech Europe \\ University Campus STeP Ri \\ Slavka Krautzeka 83/A \\ 51000 Rijeka, Croatia \\ Phone: +385 (51) 770447 \\ Fax: +385 (51) 686166 \\ www.intechopen.com
}

\author{
InTech China \\ Unit 405, Office Block, Hotel Equatorial Shanghai \\ No.65, Yan An Road (West), Shanghai, 200040, China \\ 中国上海市延安西路65号上海国际贵都大饭店办公楼405单元 \\ Phone: +86-21-62489820 \\ Fax: +86-21-62489821
}


(C) 2011 The Author(s). Licensee IntechOpen. This chapter is distributed under the terms of the Creative Commons Attribution-NonCommercialShareAlike-3.0 License, which permits use, distribution and reproduction for non-commercial purposes, provided the original is properly cited and derivative works building on this content are distributed under the same license. 